\title{
Homogenization Approach to Water Transport in Plant Tissues with Periodic Microstructures
}

\author{
A. Chavarría-Krauser ${ }^{1}$, M. Ptashnyk Pt3 $^{2,3}$ \\ ${ }^{1}$ Center for Modelling and Simulation in the Biosciences \& Interdisciplinary Center for Scientific \\ Computing, Universität Heidelberg, INF 368, 69120 Heidelberg, Germany \\ ${ }^{2}$ Department of Mathematics, University of Dundee, Old Hawkhill, Dundee DD1 4HN Scotland, UK \\ ${ }^{3}$ Ya. S. Pidstryhach Institute for Applied Problems of Mechanics and Mathematics \\ Naukova 3b, Lviv, Ukraine
}

\begin{abstract}
Water flow in plant tissues takes place in two different physical domains separated by semipermeable membranes: cell insides and cell walls. The assembly of all cell insides and cell walls are termed symplast and apoplast, respectively. Water transport is pressure driven in both, where osmosis plays an essential role in membrane crossing. In this paper, a microscopic model of water flow and transport of an osmotically active solute in a plant tissue is considered. The model is posed on the scale of a single cell and the tissue is assumed to be composed of periodically distributed cells. The flow in the symplast can be regarded as a viscous Stokes flow, while Darcy's law applies in the porous apoplast. Transmission conditions at the interface (semipermeable membrane) are obtained by balancing the mass fluxes through the interface and by describing the protein mediated transport as a surface reaction. Applying homogenization techniques, macroscopic equations for water and solute transport in a plant tissue are derived. The macroscopic problem is given by a Darcy law with a force term proportional to the difference in concentrations of the osmotically active solute in the symplast and apoplast; i.e. the flow is also driven by the local concentration difference and its direction can be different than the one prescribed by the pressure gradient.
\end{abstract}

Keywords and phrases: plant tissues, osmotic pressure, water flow, homogenisation, twoscale convergence, flows in porous media

Mathematics Subject Classification: 35B27, 35K61, 74Qxx, 76M50, 76S05, 76D07

\section{Introduction}

Plant tissues are in general composed of two domains separated by selective membranes: apoplast and symplast. The apoplast is composed of cell walls and intercellular spaces, while the symplast is constituted by all protoplasts which can be connected by plasmodesmata. Therefore, the path of water and solutes is threefold: apoplastic, symplastic and transcellular, [35,36]. A first quantitative model of water transport in plants was proposed by van den Honert, $[18,41]$. The idea was to describe water flow in analogy to the flow of electric current through a resistor network. This phenomenological approach is still contemporary,

${ }^{*}$ Corresponding author. E-mail: mptashnyk@maths.dundee.ac.uk 


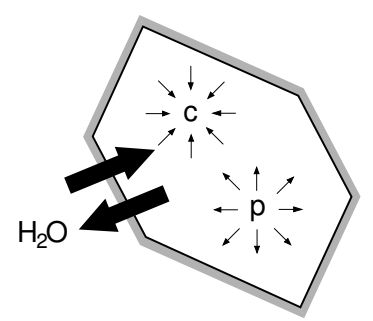

$[\mu]=0, j \cdot n=0$

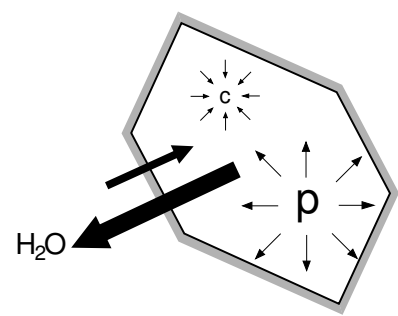

$[\mu]<0, \quad j \cdot n>0$

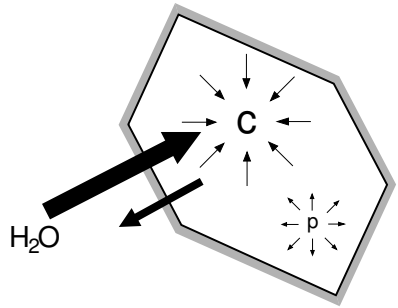

$[\mu]>0, \quad j \cdot \mathrm{n}<0$

Figure 1. Scheme of water flux into and out of a single cell. The flux is proportional to the jump in chemical potential $[\mu]$, which depends on internal pressure $p$ and solute concentration $c$.

[31], and is also used in engineering to describe water supply networks. Pressure assumes the role of the electric potential and, hence, pressure gradients produce a flux proportional to the hydraulic conductivity. This pressure driven flux was extended to include osmotically driven fluxes (diffusional fluxes) and the concept of water potential was introduced, [31]. The general concept adapted from nonequilibrium thermodynamics is that, differences in water potential produce equilibrating forces which drive the water fluxes, [40]. This relation will be presented below in more detail. Plant biologists have used this concept to describe water uptake of single cells (Fig. 1), e.g. during cell expansion [31].

Besides van den Honert's approach, less has been undertaken to extend and apply the concept for whole tissues. An interesting question is how the concepts should be used in continuum models of tissues. As will become clear later, the central problem is to find suitable transmission conditions, which describe the fluxes through the plasma membranes, and thus, between the apoplast and symplast. Another interesting task is to obtain simplified models for situations where the cell scale is small compared to the tissue or organ considered. Take for example functional structural plant models, in which often macroscopic sections of organs are combined and simulated, [43]. Whilst less complexity is the principle of a simplified model, sufficient information on the microstructure should still be part of it. Fortunatelly, plant tissues tend to be sufficiently periodic and periodic homogenization lends to treat the problem, [9].

A model of water and solute fluxes in plant tissues needs a precise description of transport processes between the cell inside (symplast) and the cell wall (apoplast) (Fig. 2). This transition takes place through semipermeable membranes and represents the connection between two different physical domains. These domains are the porous cell wall described best by a Darcy law and the cell cytoplasm in which a viscous flow applies. One of the main mathematical problems is that, in contrast to [4,21], the free fluid and porous media domains do not interact directly, as the membrane separates the domains and controls actively and passively the fluxes of water and solutes. Thus the continuity of normal forces and the Beavers-Joseph-Saffman transmission condition between free fluid and porous medium do not apply. A similar situation to the one here is found in models of early atherosclerotic lesions [5] or low-density lipoproteins transfer through arterial walls, [32,37]. Kedem-Katchalsky transmission conditions are used by those authors to couple the Navier-Stokes equations for blood flow in the arterial lumen with a Darcy law in the arterial wall. However, the dependence of fluid flow across the membrane on the oncotic pressure difference - proportional to the jump in lipoproteins concentration - was neglected by those authors to simplify the analysis of the model equations. Besides, transport of the solute in plant cells is mediated by proteins and can take place opposite to the gradient in chemical potential by usage of energy (e.g. ATPase pumps) and has to be based on a surface reaction mechanism.

Coupled free fluid and porous media problems have received an increasing attention during the last years from the mathematical and the numerical point of view. Well-posedness analysis and numerical 
algorithms for coupled Stokes-Darcy and Navier-Stokes-Darcy problems with Beavers-Joseph-Saffman transmission condition between free fluid and porous medium were addressed in $[15,25,34]$ and references therein. Along many results on the homogenization of Stokes and Navier-Stokes problems and derivation of Darcy law, $[3,19,21,26,38]$, the multiscale analysis for a Stokes-Darcy system modeling water flow in a vuggy porous media with Beavers-Joseph-Saffman transmission condition was considered in [4]. The macroscopic equations were derived using formal asymptotic expansion and two-scale convergence method. A formal asymptotic expansion was also applied successfully to define the transport velocity of auxin in a plant tissue, [7].

New transmission conditions at the cell-membrane-cell wall interface and the coupling between the flow velocity and solute concentrations via transmission conditions reflecting the osmotic nature of the water flow through a semipermeable membrane distinguish our model from the problem studied in [4]. Additional technical difficulties are introduced due to the distinction between symplastic and apoplastic velocities and the presence of plasmodesmata populated cell wall pieces. To show the existence of a unique solution of the microscopic model we apply the abstract theory of mixed problems (saddle-point problems), $[17,25]$, where the coercivity in the divergence-free space and the inf-sup condition ensure the existence of a unique solution of the coupled Stokes-Darcy model. The methods of the two-scale convergence and unfolding operator are used to derive macroscopic equations for the fluid flow and for the transport of osmotic active solutes. A generalized Darcy law with a force term given by the difference of solute concentrations in apoplast and symplast defines the macroscopic water velocity. Two initial-boundary-value problems for the concentration of solutes in symplast and in apoplast, respectively, coupled via ordinary differential equations for the transporter concentrations, describe the dynamics of solute concentrations in a plant tissue.

The paper is organized as follows. A thorough introduction to non-advective water fluxes is given. These concepts are then used to derive a biophysical model for transport of water and osmotically active solutes through a cell membrane, and to obtain transmission conditions between the symplast and apoplast (Section 2). Based on this biophysical model, a microscopic model for transport in a plant tissue is formulated (Section 3). Well-posedness and a priori estimates for solutions of the microscopic model are shown (Section 4), followed by derivation of averaged macroscopic equations for water and solute transport defined on the scale of a plant tissue (Section 5). At last, some results on two-scale convergence and periodic unfolding method are formulated (Appendix).

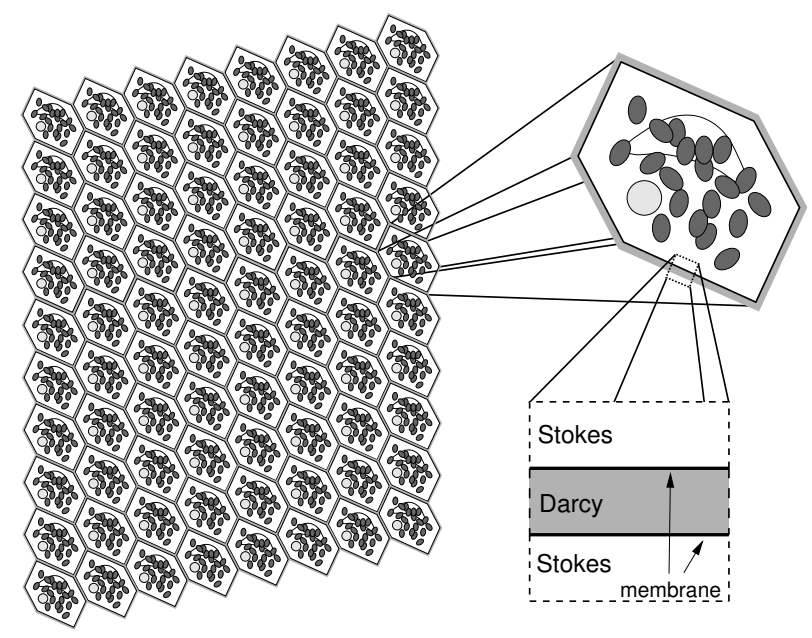

FIgURE 2. Scheme of a typical periodic plant tissue, for example lamellae of the moss Plagiomnium affine. 


\subsection{Water fluxes and chemical potential}

As noted above, water fluxes in cells are known to be driven by gradients in chemical potential, [31]. Biologist use the concept of water potential $\Psi$, which is the chemical potential of water per unit specific volume - i.e. expressed in pressure units instead of energy per particle. The chemical potential $\mu_{i}$ of a species $i$ is a measure of how much the internal energy of a thermodynamical system changes when the number of particles of species $i$ is varied, [23]. In equilibrium, the entropy of the system is maximal and consequently the temperature $T$, pressure $p$ and the chemical potentials $\mu_{i}$ are constant in abscence of external fields. Consider now a situation in which the system is not in equilibrium. In this case $\mu_{i}$ will not be constant and diffusion fluxes arise, which drive the system into equilibrium. If the system is near to equilibrium, the diffusion fluxes can be assumed to be proportional to the gradients in chemical potentials $\nabla \mu_{i}$, [24]. Irreversible thermodynamics proposes that for small gradients, the diffusion mass flux density $\boldsymbol{j}_{i}$ of a species $i$ is given by a weighted sum of the gradients of all chemical potentials.

Diffusion is a spontaneous molecular equilibration process which does not produce bulk flows in closed systems. The so called mass constraint applies when the system is not divided by a membrane, [16]

$$
\boldsymbol{j}=\sum_{i} \boldsymbol{j}_{i}=0,
$$

which states that diffusion cannot produce a macroscopic movement of the mixture. Condition (1.1) is not valid when the domain is separated by a semipermeable membrane, as such membranes allow the movement of the solvent but not of all solutes. A consequence is that in such a case the system tends to a local equilibrium: entropy reaches a local maximum and removing the membrane would allow a further increase. To account for semipermeable membranes, the concepts were extended to include a so called reflection coefficient, [31]. This coefficient is a measure of how much solutes are reflected by the membrane. A value of one means that all solutes are retained, while all solutes cross freely for a value of zero. The reflection coefficient lies in general between 0 and 1 for a real membrane, [31].

For the sake of simplicity, we will consider here and in the sequel the case of a binary mixture of a solvent $(i=1)$ and a solute $(i=2)$. Following the notation of [24], we introduce the mass fraction of the solute as a concentration

$$
c:=\rho_{2} / \rho,
$$

where $\rho_{i}$, for $i=1,2$ is the mass density of species $i$ and $\rho=\rho_{1}+\rho_{2}$ the mass density of the mixture. Consequently the "concentration" of the solvent is $1-c$. This duality of the concentrations allows to introduce one chemical potential

$$
\mu=\frac{\mu_{2}}{m_{2}}-\frac{\mu_{1}}{m_{1}},
$$

instead of two, [24], where $m_{i}$ is the mass of one particle of species $i$, for $i=1,2$. Note that $m_{1}$ and $m_{2}$ are needed to obtain a chemical potential density in units energy per mixture mass. The flux density of the solute is then $\boldsymbol{j}_{2}=-\alpha \nabla \mu$ for $\alpha>0$. Using the mass constraint (1.1), delivers for that case the flux density $\boldsymbol{j}_{1}=\alpha \nabla \mu$ of the solvent. The combined chemical potential cannot be used in the case of a semipermeable membrane and the approach needs to be extended by a reflection coefficient. For this purpose, we introduce two diffusion driving potentials

$$
\begin{aligned}
& \tilde{\mu}_{1}:=\frac{\mu_{1}}{m_{1}}-(1-\varsigma) \frac{\mu_{2}}{m_{2}} \quad \text { and } \quad \tilde{\mu}_{2}:=(1-\varsigma)\left(\frac{\mu_{2}}{m_{2}}-\frac{\mu_{1}}{m_{1}}\right), \\
& \tilde{\mu}_{1}=-(1-\varsigma) \mu+\varsigma \frac{\mu_{1}}{m_{1}} \quad \text { and } \quad \tilde{\mu}_{2}=(1-\varsigma) \mu,
\end{aligned}
$$

where $0 \leq \varsigma \leq 1$ is a reflection coefficient. Setting $\varsigma=0$ renders $\tilde{\mu}_{2}=-\tilde{\mu}_{1}=\mu$, which is consistent with [24], while setting $\varsigma=1$ gives $\tilde{\mu}_{1}=\mu_{1} / m_{1}$ and $\tilde{\mu}_{2}=0$. The corresponding mass flux densities are simply

$$
\begin{aligned}
\boldsymbol{j}_{i} & =-\alpha \nabla \tilde{\mu}_{i}, \quad \text { for } i=1,2, \\
\boldsymbol{j} & =-\alpha \nabla\left(\tilde{\mu}_{1}+\tilde{\mu}_{2}\right)=-\alpha \varsigma \nabla \mu_{1},
\end{aligned}
$$


where $\alpha>0$ is a coefficient related to the permeability towards the solvent. The expression for $\boldsymbol{j}$ shows that reflection $(\varsigma \neq 0)$ is a must to produce bulk fluxes, and is actually the mechanism exploited by plant cells, [31]. Figure 1 presents a scheme of water fluxes through a membrane of a single cell with a semipermeable membrane $\left(\varsigma=1, \tilde{\mu}_{2}=0\right.$ and $\left.\boldsymbol{j}_{2}=0\right)$.

Often in mathematical models, diffusion is assumed to be driven only by concentration gradients, which is equivalent to setting $\tilde{\mu}_{2}=\tilde{\mu}_{2}(c)$. For this case, Eqs. (1.5) deliver Fick's law with reflection, [24]

$$
\boldsymbol{j}_{2} \approx-\rho(1-\varsigma) D \nabla c
$$

where $D>0$ is the usual diffusion coefficient [see Eq. (1.8)]. This approach ignores that the chemical potential depends also on pressure $p$ and temperature $T$, which implies that gradients in $p$ and $T$ produce also fluxes. This dependency is normally assumed to be small, although the pressure term is known to be important in sedimentation processes, [42], and in cases where the pressure is nonharmonic and concentration gradients are small, [6]. Fick's law (1.6) is a fairly good approximation in a homogeneous domain, but is inapplicable for obtaining transmission conditions at semipermeable interfaces. Large pressure differences can arise across a membrane and the contributions of pressure and concentration driven diffusion are of comparable magnitude.

To account for pressure driven diffusion, the potentials $\tilde{\mu}_{i}$ are set to depend on concentration and pressure. For simplicity, the dependence on temperature is neglected, as large temperature gradients are not usual in plant tissues. Eqs. (1.5) render

$$
\begin{aligned}
\boldsymbol{j}_{2} & =-\rho(1-\varsigma)(D \nabla c+G \nabla p), \\
\boldsymbol{j} & =\rho \varsigma(\mathcal{D} \nabla c-\mathcal{G} \nabla p) \quad \text { and } \\
\boldsymbol{j}_{1} & =-\boldsymbol{j}_{2}+\boldsymbol{j},
\end{aligned}
$$

where

$$
D=\frac{\alpha}{\rho} \frac{\partial \mu}{\partial c}>0, \quad G=\frac{\alpha}{\rho} \frac{\partial \mu}{\partial p}, \quad \mathcal{D}=-\frac{\alpha}{\rho} \frac{\partial \mu_{1}}{\partial c}>0, \quad \mathcal{G}=\frac{\alpha}{\rho} \frac{\partial \mu_{1}}{\partial p}>0 .
$$

The diffusion coefficients $D$ and $\mathcal{D}$ are positive, while the barodiffusion coefficient $G$ has no definite sign, [24]. Assuming incompressibility of the solvent has as a consequence that $\mathcal{G}$ is positive, [31]. These signs concord with what is known from biology: diffusion fluxes across the mebrane follow the concentration gradient and are oriented against the pressure gradient (see Fig. 1).

\section{Biophysical model}

\subsection{Mass conservation}

The total flux density of a species and the mixture is given by the combination of the contributions of advection $\rho_{i} \boldsymbol{v}$, diffusion $\boldsymbol{j}_{i}$ and membrane transport via transporting proteins $\boldsymbol{a}_{i}$

$$
\begin{aligned}
\boldsymbol{J}_{i} & =\rho_{i} \boldsymbol{v}+\boldsymbol{j}_{i}+\boldsymbol{a}_{i}, \quad i=1,2, \\
\boldsymbol{J} & =\rho \boldsymbol{v}+\boldsymbol{j}+\boldsymbol{a}
\end{aligned}
$$

where $\boldsymbol{j}:=\sum_{i} \boldsymbol{j}_{i}$ and $\boldsymbol{a}:=\sum_{i} \boldsymbol{a}_{i}$. Consequently, conservation of the species and total mass are given by

$$
\begin{aligned}
\partial_{t} \rho_{i}+\operatorname{div}\left(\rho_{i} \boldsymbol{v}+\boldsymbol{j}_{i}+\boldsymbol{a}_{i}\right) & =0, \quad i=1,2, \\
\partial_{t} \rho+\operatorname{div}(\rho \boldsymbol{v}+\boldsymbol{j}+\boldsymbol{a}) & =0 .
\end{aligned}
$$




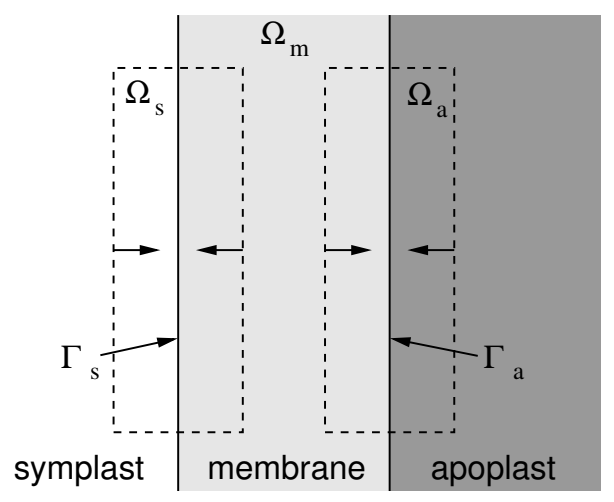

FiguRE 3. Scheme of the method used to obtain the transmission conditions. Small domains $\Omega_{s}$ and $\Omega_{a}$ enclosing the interface symplast-membrane and membrane-apoplast, respectively, are reduced to the surfaces $\Gamma_{s}$ and $\Gamma_{a}$. The membrane is represented by the domain $\Omega_{m}$.

First, consider the laws in a compartment without an interface. Transport via proteins takes place only on membranes. Therefore, $\boldsymbol{a}_{i}$ are zero inside a domain $\Omega$ that does not contain a membrane, and the mass constraint (1.1) applies. Conservation of solute and mass in $\Omega$ follows thus

$$
\begin{aligned}
\partial_{t} \rho_{i}+\operatorname{div}\left(\rho_{i} \boldsymbol{v}+\boldsymbol{j}_{i}\right)=0 & & \text { in } \Omega \times(0, \infty), & i=1,2, \\
\partial_{t} \rho+\operatorname{div}(\rho \boldsymbol{v})=0 & & \text { in } \Omega \times(0, \infty) . &
\end{aligned}
$$

Consider now the case where a membrane is present. Shall $\Omega_{s}$ be a domain enclosing an arbitrary piece $\Gamma_{s}$ of the interface between the symplast and the membrane (Fig. 3). Shall the membrane be represented by the domain $\Omega_{m}$. Note that the derivation of the conditions for the interface between membrane and apoplast is conducted in the same manner and will not be given here explicitly. Because of the membrane, $\varsigma \neq 0$ and $\boldsymbol{a}_{i} \neq 0$ for $i=1,2$. Integration of (2.2a) over $\Omega_{s}$ and application of Gauß' law gives

$$
\partial_{t} \int_{\Omega_{s}} \rho_{i} d x+\int_{\partial \Omega_{s}}\left(\rho_{i} \boldsymbol{v}+\boldsymbol{j}_{i}+\boldsymbol{a}_{i}\right) \cdot \boldsymbol{n} d \gamma=0 .
$$

The membrane can be assumed to not allow advective fluxes, so that on $\partial \Omega_{s} \cap \Omega_{m}$ we have $\boldsymbol{v}=0$. On $\partial \Omega_{s} \backslash \bar{\Omega}_{m}$ there is no protein mediated transport so that $\boldsymbol{a}=0$ there. The thickness of $\Omega_{s}$ is reduced to zero such that the interface is kept inside $\left(\Omega_{s} \rightarrow \Gamma_{s}\right)$. The first integral tends to zero, while the second tends to an integral over $\Gamma_{s}$

$$
\int_{\Gamma_{s}}\left(-\rho_{i} \boldsymbol{v}+\overline{\boldsymbol{j}}_{i}-\boldsymbol{j}_{i}+\boldsymbol{a}_{i}\right) \cdot \boldsymbol{n} d \gamma=0,
$$

where $\overline{\boldsymbol{j}}_{i}$ is the diffusion flux in $\Omega_{m}$. Note that $\boldsymbol{n}$ points from the symplast to the apoplast. Because $\Omega_{s}$ and $\Gamma_{s}$ were chosen arbitrarily, the integrand has to be zero. A similar approach can be applied to the mass flux of the mixture, where $\boldsymbol{j}=0$ in $\Omega$ is used. We obtain the conditions

$$
\begin{aligned}
\left(\rho_{i} \boldsymbol{v}+\boldsymbol{j}_{i}\right) \cdot \boldsymbol{n} & =\left(\boldsymbol{a}_{i}+\overline{\boldsymbol{j}}_{i}\right) \cdot \boldsymbol{n} & & \text { on }\left(\Gamma_{s} \cup \Gamma_{a}\right) \times(0, \infty), \\
\rho \boldsymbol{v} \cdot \boldsymbol{n} & =(\boldsymbol{a}+\overline{\boldsymbol{j}}) \cdot \boldsymbol{n} & & \text { on }\left(\Gamma_{s} \cup \Gamma_{a}\right) \times(0, \infty),
\end{aligned}
$$

where $\overline{\boldsymbol{j}}=\overline{\boldsymbol{j}}_{1}+\overline{\boldsymbol{j}}_{2}$. The fluxes in $\Omega$ were written on the left hand side, while the fluxes in $\Omega_{m}$ are on right hand side. The diffusion fluxes $\overline{\boldsymbol{j}}_{i}$ are normally assumed to be constant in the membrane (constant gradients), [31]. Protein mediated transport can be considered to be a chemical reaction allowing buffering of species, and hence, the fluxes $\boldsymbol{a}_{i, \mathrm{I}}$ and $\boldsymbol{a}_{i, \mathrm{II}}$ through $\Gamma_{s}$ and $\Gamma_{a}$, respectively, have not necessarily to be equal. Expressions for $\boldsymbol{a}_{i, \mathrm{I}}$ and $\boldsymbol{a}_{i, \mathrm{Il}}$ will be developed in Section 2.2. 


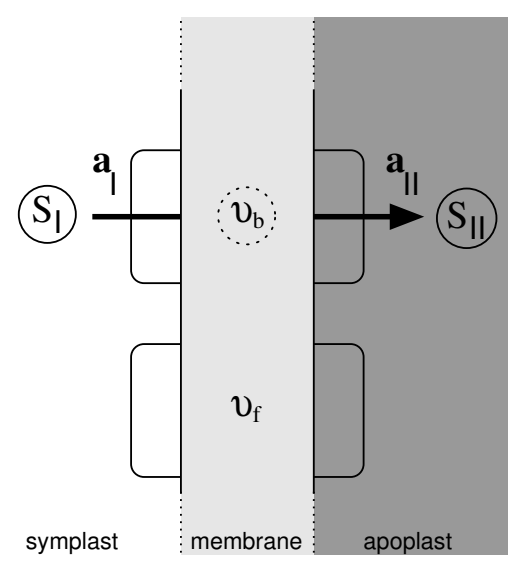

FIGURE 4. Depiction of the mechanism assumed for protein mediated transport, where a transport direction from symplast to apoplast is shown exemplarily.

The membrane $\Omega_{m}$ is very thin compared to the rest and is usually reduced to an interface denoted here as $\Gamma_{m}$. An expression for the membrane's buffering effect is obtained by integration of (2.2a) over $\Omega_{m}$, application of Gauß' law and reduction of $\Omega_{m}$ to $\Gamma_{m}$. This process is equivalent to subtraction of the transmission conditions on $\Gamma_{s}$ from those on $\Gamma_{a}$. Finally, the transmission conditions on the reduced membrane interface $\Gamma_{m}$ are in total

$$
\begin{aligned}
& \left(\rho_{i} \boldsymbol{v}+\boldsymbol{j}_{i}\right) \cdot \boldsymbol{n}=\left(\boldsymbol{a}_{i, \mathrm{I}}+\overline{\boldsymbol{j}}_{i}\right) \cdot \boldsymbol{n} \quad \text { on } \Gamma_{m} \times(0, \infty), \quad i=1,2 \\
& \rho \boldsymbol{v} \cdot \boldsymbol{n}=\left(\boldsymbol{a}_{1}+\overline{\boldsymbol{j}}\right) \cdot \boldsymbol{n} \quad \text { on } \Gamma_{m} \times(0, \infty), \\
& {\left[\rho_{i} \boldsymbol{v}+\boldsymbol{j}_{i}\right] \cdot \boldsymbol{n}=\left[\boldsymbol{a}_{i}\right] \cdot \boldsymbol{n} \quad \text { on } \Gamma_{m} \times(0, \infty), \quad i=1,2,} \\
& {[\rho \boldsymbol{v}] \cdot \boldsymbol{n}=[\boldsymbol{a}] \cdot \boldsymbol{n} \quad \text { on } \Gamma_{m} \times(0, \infty),}
\end{aligned}
$$

where the assumptions $\overline{\boldsymbol{j}}_{i}=$ const, and $\overline{\boldsymbol{j}}=$ const in the membrane were used, and [.] is defined as the jump across the membrane (i.e. $\left[\boldsymbol{a}_{i}\right]=\boldsymbol{a}_{i, \mathrm{Il}}-\boldsymbol{a}_{i, 1}$ ).

\subsection{Protein mediated transport}

Most solute fluxes through membranes are mediated by transporting proteins, [31]. Transport can be either passive or active with usage of energy (e.g. ATPase pumps). We model this processes as surface reactions, in which the solute on one side $\left(S_{\mathrm{I}}\right)$ reacts with the transporter, builds a complex $(S-T)$, which decays by transporting the solute to the other side $\left(S_{\mathrm{II}}\right)$

$$
S_{\text {। }}+T \underset{k_{2}}{\stackrel{k_{1}}{\rightleftharpoons}} S-T \underset{k_{4}}{\stackrel{k_{3}}{\rightleftharpoons}} S_{\text {।I }}+T .
$$

The concentrations of the solute on either sides $\left(S_{\mathrm{I}}\right.$ and $\left.S_{\mathrm{II}}\right)$ are denoted by $c_{\mathrm{I}}$ and $c_{\mathrm{II}}$, respectively. The concentrations of free and bound transporters are denoted with $\vartheta_{f}$ and $\vartheta_{b}$, respectively. In general, the density of the mixture can differ between sides and we denote these here with $\rho_{\mathrm{l}}$ and $\rho_{\| I}$. The above simple reaction mechanism produces fluxes on each side

$$
\begin{aligned}
& \boldsymbol{a}_{\mathbf{l}}=\left(k_{1} \rho_{\mathbf{l}} c_{\mathbf{l}} \vartheta_{f}-k_{2} \vartheta_{b}\right) \boldsymbol{n}, \\
& \boldsymbol{a}_{\| \mathbf{I I}}=\left(k_{3} \vartheta_{b}-k_{4} \rho_{\text {|I }} c_{||} \vartheta_{f}\right) \boldsymbol{n} \text {, }
\end{aligned}
$$

where $\boldsymbol{n}$ is the normal of the membrane pointing out of the cell. These expressions correspond to the rate law of the above reaction mechanism. See Fig. 4 for a depiction of this mechanism. 
The concentration of free and bound transporters are obtained by pointwise reactions. The rate of change of free transporters due to binding of solute and decay of the complex is given by

$$
[\boldsymbol{a}] \cdot \boldsymbol{n}=\left(\boldsymbol{a}_{\mathrm{II}}-\boldsymbol{a}_{\mathrm{I}}\right) \cdot \boldsymbol{n}=-\left(k_{1} \rho_{\mathrm{I}} c_{\mathrm{I}}+k_{4} \rho_{\mathrm{II}} c_{\mathrm{II}}\right) \vartheta_{f}+\left(k_{2}+k_{3}\right) \vartheta_{b} .
$$

The net transport direction varies in time and depends on the solute concentrations and coefficients $k_{1}$ to $k_{4}$. By focusing on a quasistationary situation in which $\boldsymbol{a}_{\|}=\boldsymbol{a}_{\mathrm{l}}:=\boldsymbol{a}$, the following net flux and concentration of free transporters are obtained

$$
\begin{aligned}
\boldsymbol{a} & =\left(k_{1} k_{3} \rho_{\mathrm{I}} c_{\mathrm{I}}-k_{2} k_{4} \rho_{\mathrm{II}} c_{\mathrm{II}}\right) /\left(k_{2}+k_{3}\right) \vartheta_{f} \boldsymbol{n}, \\
\vartheta_{f} & =\left(k_{2}+k_{3}\right) /\left(k_{2}+k_{3}+k_{1} \rho_{\mathrm{I}} c_{\mathrm{I}}+k_{4} \rho_{\mathrm{II}} c_{\mathrm{II}}\right) \vartheta_{0},
\end{aligned}
$$

where $\vartheta_{0}(t, x)=\vartheta_{f}(t, x)+\vartheta_{b}(t, x)$ is the total amount of transporters. We will focus here on the special case where either $k_{1}=0$ or $k_{4}=0$, i.e. on the case of a perfect uni-directional transporter. Using the assumption of quasistationarity, the mechanism is found to follow Michaelis-Menten kinetics. Exemplary for an efflux transporter $\left(k_{4}=0\right)$

$$
\boldsymbol{a}=\rho_{\mathbf{l}} c_{\mathrm{l}} k_{1} k_{3} /\left(k_{2}+k_{3}+k_{1} \rho_{\mathrm{I}} c_{\mathrm{l}}\right) \boldsymbol{n} .
$$

The applicability of the assumption of quasistationarity is limited. Transporters are in general subjected to regulation, which reduces or increases the amount of transporters depending on the status of the system. The total amount of transporters varies as follows

$$
\partial_{t} \vartheta_{0}=R\left(t, x, \vartheta_{0}\right)
$$

where $R\left(t, x, \vartheta_{0}\right)$ is a function describing the rate of regulation. $R$ is in general composed of a positive production term and a negative degradation term. Negative degradation is often assumed to be proportional to $\vartheta_{0}$ to prevent negative concentrations. The production term will depend on the local or global solute concentration and regulation is used to control that concentration. A general analysis of robust homeostatic control of a species based on concentrations is presented in [30], and an example for zinc homeostasis in yeast and plant cells is found in [11-13]. We will not consider details of regulation here.

Assume that only free transporters are regulated and by using Eq. (2.7), the following system describing the pointwise dynamics of the free and bound transporters is obtained

$$
\begin{aligned}
& \partial_{t} \vartheta_{f}=R\left(t, x, \vartheta_{f}\right)-\left(k_{1} \rho_{\mathrm{I}} c_{\mathrm{I}}+k_{4} \rho_{\mathrm{II}} c_{\mathrm{II}}\right) \vartheta_{f}+\left(k_{2}+k_{3}\right) \vartheta_{b}, \\
& \partial_{t} \vartheta_{b}=\quad\left(k_{1} \rho_{\mathrm{I}} c_{\mathrm{I}}+k_{4} \rho_{\mathrm{II}} c_{\mathrm{II}}\right) \vartheta_{f}-\left(k_{2}+k_{3}\right) \vartheta_{b} \text {, } \\
& \vartheta_{0}(t, x)=\vartheta_{f}(t, x)+\vartheta_{b}(t, x) \text {. }
\end{aligned}
$$

In a general situation several influx and efflux transporters might exist. The fluxes generalize then into

$$
\boldsymbol{a}_{i, \mathrm{I}}=\sum_{\alpha}\left(k_{1, \alpha}^{i} \rho_{i, \mathrm{I}} \vartheta_{f, \alpha}^{i}-k_{2, \alpha}^{i} \vartheta_{b, \alpha}^{i}\right) \boldsymbol{n}, \quad \text { and } \quad \boldsymbol{a}_{i, \mathrm{I}}=\sum_{\alpha}\left(k_{3, \alpha}^{i} \vartheta_{b, \alpha}^{i}-k_{4, \alpha}^{i} \rho_{i, \mathrm{I}} \vartheta_{f, \alpha}^{i}\right) \boldsymbol{n}
$$

where $\rho_{i}=\rho c_{i}$, with $i=1,2$, and all $\vartheta_{f, \alpha}^{i}$ and $\vartheta_{b, \alpha}^{i}$ fulfill an equation system equivalent to Eq. (2.11).

\subsection{Model}

Inserting the diffusion fluxes of Eq. (1.7) into Eqs. (2.3a) and (2.3b), and using $\rho_{2}=\rho c$ delivers a system describing species and mass conservation in a compartment $\Omega$ with $\varsigma=0$

$$
\begin{aligned}
\partial_{t}(\rho c)+\operatorname{div}(\rho c \boldsymbol{v}-\rho D \nabla c-\rho G \nabla p)=0 & \text { in } \Omega \times(0, \infty) \\
\partial_{t} \rho+\operatorname{div}(\rho \boldsymbol{v})=0 & \text { in } \Omega \times(0, \infty)
\end{aligned}
$$


The gradients in the membrane can be assumed to be constant and can be expressed by a jump across the membrane. Therefore, the diffusion fluxes in the membrane $\overline{\boldsymbol{j}}_{2}$ and $\overline{\boldsymbol{j}}$ are functions of the jumps in concentration and pressure

$$
\overline{\boldsymbol{j}}_{2}([c],[p])=-\frac{\rho}{h}(D[c]+G[p]) \boldsymbol{n}, \quad \text { and } \quad \overline{\boldsymbol{j}}([c],[p])=\frac{\rho}{h} \varsigma(\mathcal{D}[c]-\mathcal{G}[p]) \boldsymbol{n},
$$

where the gradients in Eqs. (1.7) were approximated by a jump across the membrane of thickness $h$. The expressions in Eq. (2.14) are related to the Kedem-Katchalsky equations, [5].

Using Eqs. (1.7) in (2.5a) and (2.5c), the transmission conditions on the interface $\Gamma_{m}$ for the solute are obtained

$$
\begin{array}{ll}
(\rho c \boldsymbol{v}-\rho D \nabla c-\rho G \nabla p) \cdot \boldsymbol{n}=\left(\boldsymbol{a}_{2, I}+\overline{\boldsymbol{j}}_{2}([c],[p])\right) \cdot \boldsymbol{n} & \text { on } \Gamma_{m} \times(0, \infty), \\
{[\rho c \boldsymbol{v}-\rho D \nabla c+\rho G \nabla p] \cdot \boldsymbol{n}=\left[\boldsymbol{a}_{2}\right] \cdot \boldsymbol{n}} & \text { on } \Gamma_{m} \times(0, \infty),
\end{array}
$$

where $\boldsymbol{a}_{2}$ and $\overline{\boldsymbol{j}}_{2}$ are given by Eqs. (2.12) and (2.14), respectivelly.

Water is almost incompressible $\left(\rho_{1} \approx\right.$ const $)$ and compressibility arises here due to the solute influencing the mixture's density $\rho=\rho_{1}+\rho_{2}=(1-c) \rho+c \rho$. Using the assumption of a small concentration $c$, in a first approximation $\rho \approx \rho_{1} \approx$ const. Also, barodiffusion in $\Omega$ can be assumed to be small compared to the concentration driven flux, i.e. it is assumed to play only a role in transport through the membrane. Viscosity can be assumed to dominate the flow due to the small characteristic scale of a few microns, and the flow in the symplast (cell inside) is assumed to be a Stokes flow. The apoplast (cell wall) is porous suggesting a Darcy flow. The equations describing flow, mass and species conservation are hence

$$
\begin{aligned}
\partial_{t} \boldsymbol{v}-\frac{\eta}{\rho_{1}} \Delta \boldsymbol{v}+\frac{1}{\rho_{1}} \nabla p & =0 & & \text { in symplast, } \\
\boldsymbol{v}+K \nabla p & =0 & & \text { in apoplast, } \\
\operatorname{div} \boldsymbol{v} & =0 & & \text { in symplast and apoplast, } \\
\partial_{t} c+\operatorname{div}(\boldsymbol{v} c-D \nabla c) & =0 & & \text { in symplast and apoplast, }
\end{aligned}
$$

where $\eta>0$ is the dynamic viscosity and $K>0$ is the permeability of the apoplast.

Diffusive permeation of the solute can be assumed to be small compared against protein mediated transport. We will also assume that only the solute is subjected to transport via proteins. Assuming that the structure in the membrane is oriented normally, the velocity at the interface can be assumed to be perpendicular to it $(\boldsymbol{v} \times \boldsymbol{n}=0)$. Together with incompressibility, this condition has as a consequence that the normal viscous stress $\left(\boldsymbol{\sigma}^{\prime} \boldsymbol{n}\right) \cdot \boldsymbol{n}$ is zero on a flat boundary, i.e. the boundary experiences only shear stress. The viscous stress tensor of an incompressible viscous fluid is defined as

$$
\boldsymbol{\sigma}^{\prime}=2 \eta \mathrm{S} \boldsymbol{v}
$$

with $\mathrm{S} \boldsymbol{v}$ the symmetric velocity gradient

$$
\mathrm{S} \boldsymbol{v}=\frac{1}{2}\left(\nabla \boldsymbol{v}+\nabla \boldsymbol{v}^{\top}\right)
$$

and is related to the full stress tensor $\boldsymbol{\sigma}=-p \boldsymbol{I}+\boldsymbol{\sigma}^{\prime}=-p \boldsymbol{I}+2 \eta \mathrm{S} \boldsymbol{v}$. Assuming that the effect of corners is small, it is possible to set $\left(\boldsymbol{\sigma}^{\prime} \boldsymbol{n}\right) \cdot \boldsymbol{n}=0$ on $\Gamma_{m} \times(0, \infty)$. Water fluxes mediated by active water transporters are probably small compared to the fluxes via passive transporters (aquaporins): $\left\|\boldsymbol{a}_{\boldsymbol{l}}\right\| \ll\|\overline{\boldsymbol{j}}\|$. Moreover, by adding $\left(\boldsymbol{\sigma}^{\prime} \boldsymbol{n}\right) \cdot \boldsymbol{n}=0$ only on the Stokes flow side, Eq. (2.5b) becomes

$$
\kappa\left(\left(\boldsymbol{\sigma}^{\prime} \boldsymbol{n}\right) \cdot \boldsymbol{n}+[p]\right)=\delta[c]-\boldsymbol{v} \cdot \boldsymbol{n} \quad \text { on } \Gamma_{m} \times(0, \infty),
$$

where $\kappa:=\varsigma \mathcal{G} / h, \delta:=\varsigma \mathcal{D} / h$. Note that the normal and the jump [.] are oriented from the Stokes to the Darcy side. The physical meaning of this condition becomes clear by setting $\kappa=\delta=0$

$$
\boldsymbol{v} \cdot \boldsymbol{n}=0 \quad \text { for } \kappa=\delta=0 \quad \text { on } \Gamma_{m} \times(0, \infty),
$$


i.e. the net mass flux is zero for zero permeability $(\alpha=0)$ or for a nonreflecting membrane $(\varsigma=0)$. Also, a higher concentration in the cell, $[c]<0$, means that water is driven into the cell, $\boldsymbol{v} \cdot \boldsymbol{n}<0$ for $[p]=0$, while a higher pressure in the cell, $[p]<0$, results in water flowing out of the cell, $\boldsymbol{v} \cdot \boldsymbol{n}>0$ for $[c]=0$. Compare Fig. 1 .

Finally, the following conditions are good approximations on the interface $\Gamma_{m}$

$$
\begin{aligned}
\rho_{1}\left(c_{1} \boldsymbol{v}_{1}-D_{2} \nabla c_{1}\right) \cdot \boldsymbol{n} & \approx \boldsymbol{a}_{2, \mathrm{I}} \cdot \boldsymbol{n} \\
\rho_{1}\left[c \boldsymbol{v}-D_{2} \nabla c\right] \cdot \boldsymbol{n} & \approx\left[\boldsymbol{a}_{2}\right] \cdot \boldsymbol{n} \\
\kappa(2 \eta(\mathrm{S} \boldsymbol{v} \boldsymbol{n}) \cdot \boldsymbol{n}+[p]) & \approx \delta[c]-\boldsymbol{v}_{1} \cdot \boldsymbol{n} \\
{[\boldsymbol{v}] \cdot \boldsymbol{n} } & \approx 0 \\
\boldsymbol{v} \times \boldsymbol{n} & =0
\end{aligned}
$$

$$
\begin{aligned}
& \text { on } \Gamma_{m} \times(0, \infty), \\
& \text { on } \Gamma_{m} \times(0, \infty), \\
& \text { on } \Gamma_{m} \times(0, \infty), \\
& \text { on } \Gamma_{m} \times(0, \infty), \\
& \text { on } \Gamma_{m} \times(0, \infty) .
\end{aligned}
$$

\section{Mathematical formulation of microscopic model}

Let $\Omega$ be a cube in $\mathbb{R}^{3}$ representing a plant tissue, and $\varepsilon>0$ be a parameter denoting the ratio between the size of a single cell and the size of the considered plant tissue $\Omega$. The microscopic structure of a plant tissue is reflected in the difference between the cell wall $\Omega_{a}^{\varepsilon} \subset \Omega$ and the symplast inside the cells $\Omega_{z}^{\varepsilon} \subset \Omega$. In the cell wall domain we shall distinguish between the cell wall apoplast $\Omega_{a w}^{\varepsilon}$ and parts of the cell wall $\Omega_{\text {as }}^{\varepsilon}$ occupied by both plasmodesmata, that belong to cell symplast, and cell wall apoplast. This partition is a strong simplification of the true geometry of a plant tissue, however, it accounts for the basic structures. See Ref. [14] for more anatomical details of plant cells and tissues.

We define a unit cell $Y=\bar{Y}_{z} \cup \bar{Y}_{a}$, where $Y_{z}$ is an open domain with a smooth boundary, representing the part occupied by symplast inside a cell, and $Y_{a}$ is the cell wall, with $\bar{Y}_{a}=\bar{Y}_{a w} \cup \bar{Y}_{a s}$ and $Y_{a s}$ is a domain comprising both plasmodesmata and cell wall apoplast. We define also $\bar{Y}_{s}=\bar{Y}_{z} \cup \bar{Y}_{a s}$. The corresponding boundaries are denoted by $\Gamma_{z}=\partial Y_{a w} \cap \partial Y_{z}, \Gamma_{a w}=\partial Y_{a w} \cap \partial Y_{a s}, \Gamma_{a}=\partial Y_{a} \backslash \partial Y$, $\Gamma_{s}=\partial Y_{s} \backslash \partial Y, \Gamma_{a s}=\partial Y_{a s} \cap \partial Y_{z}, \Gamma_{z s}=\Gamma_{z} \cup \Gamma_{a s}=\partial Y_{z}$, see Fig. 5 .
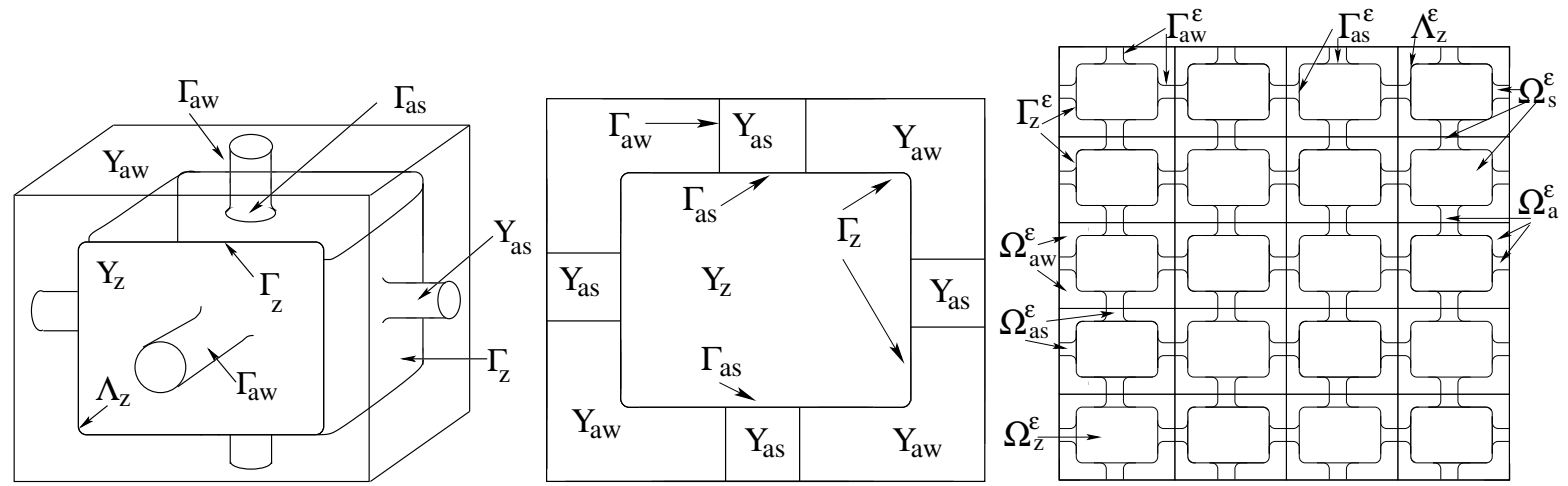

Figure 5. Left: unit cell $Y$. Center: 2D cross-section of a unit cell $Y$. Right: 2D cross-section of a plant tissue $\Omega$.

Then considering translations of the unit cell given by $Y_{j}^{k}=Y_{j}+k$ for $j=a, z, s$, aw or as, and $\Gamma_{i}^{k}=\Gamma_{i}+k$ for $i=z$, aw, as or $z s$, with $k \in \mathbb{Z}^{3}$, we can define the domains comprising the microstructure of a plant tissue $\Omega_{j}^{\varepsilon}=\cup\left\{\varepsilon Y_{j}^{k}: \varepsilon Y^{k} \subset \Omega, k \in \mathbb{Z}^{3}\right\}, j=a, z, s$, aw or as. The microscopic boundaries are given by $\Gamma_{i}^{\varepsilon}=\cup\left\{\varepsilon \Gamma_{i}^{k}: \varepsilon Y^{k} \subset \Omega, k \in \mathbb{Z}^{3}\right\}, i=z$, aw, as, or $z s$. We notice that $\Omega_{s}^{\varepsilon}$ and $\Omega_{a}^{\varepsilon}$ are connected Lipschitz domains. The domain $\Omega_{a}^{\varepsilon}$ represent porous medium of cell walls, whereas $\Omega_{s p}^{\varepsilon}$ is introduced 
to depict parts of cell walls, modelled also as a porous medium, comprising both cell wall apoplast and many thin channels of plasmodesmata, Fig. 5 .

In order to simplify the formulation of the microscopic model we shall consider in the following the doubled notation for $\Omega_{a s}^{\varepsilon}$, i.e. $\Omega_{a p}^{\varepsilon}:=\Omega_{a s}^{\varepsilon}$ and $\Omega_{s p}^{\varepsilon}:=\Omega_{a s}^{\varepsilon}$.

For the velocity of water we shall consider the stationary version of Stokes equation (2.16a) inside the cells and Darcy equation $(2.16 \mathrm{~b})$ in the cell walls apoplast and parts of cell walls comprising plasmodesmata, and incompressibility $(2.16 \mathrm{c})$ of all flows

$$
\begin{array}{rll}
-\varepsilon^{2} \eta \Delta \boldsymbol{v}_{z}^{\varepsilon}+\nabla p_{z}^{\varepsilon}=0 & \text { in } \Omega_{z}^{\varepsilon} \times(0, T), & \\
\operatorname{div} \boldsymbol{v}_{z}^{\varepsilon}=0 & \text { in } \Omega_{z}^{\varepsilon} \times(0, T), & \\
\boldsymbol{v}_{i}^{\varepsilon}+K_{i, \varepsilon} \nabla p_{i}^{\varepsilon}=0 & \text { in } \Omega_{i}^{\varepsilon} \times(0, T), & i=s p, a w, a p \\
\operatorname{div} \boldsymbol{v}_{i}^{\varepsilon}=0 & \text { in } \Omega_{i}^{\varepsilon} \times(0, T), & i=s p, a w, a p
\end{array}
$$

where $\eta>0$ is the viscosity constant, and the permeability tensors are given by $Y$-periodic functions $K_{i}$, i.e. $K_{i, \varepsilon}(x)=K_{i}(x / \varepsilon)$ for $x \in \Omega_{i}^{\varepsilon}$, with $i=s p$, aw, ap.

The concentration of an osmotically active solute in different partitions of a plant cell is assumed to follow the conservation Eq. (2.16d) with a production/degradation term

$$
\partial_{t} c_{i}^{\varepsilon}+\operatorname{div}\left(H_{M}\left(\boldsymbol{v}_{i}^{\varepsilon}\right) c_{i}^{\varepsilon}-D_{i}^{\varepsilon}(t, x) \nabla c_{i}^{\varepsilon}\right)=F_{i}^{\varepsilon}\left(t, x, c_{i}^{\varepsilon}\right) \quad \text { in } \Omega_{i}^{\varepsilon} \times(0, T), \quad i=z, s p, a w, a p,
$$

where

$$
H_{M}(\boldsymbol{\xi})_{j}=\left\{\begin{array}{ll}
\xi_{j} & \text { for }\left|\xi_{j}\right| \leq M, \\
M \operatorname{sgn}\left(\xi_{j}\right) & \text { for }\left|\xi_{j}\right|>M,
\end{array} \quad j=1,2,3,\right.
$$

for some $M>0$, and $\boldsymbol{\xi} \in \mathbb{R}^{3}$. The velocities $\boldsymbol{v}_{i}^{\varepsilon}$ need to be cut-off for technical reasons for the rigorous analysis of the macroscopic model. Assuming bounded velocities in tissues is biologically and physically sensible. The diffusion coefficients $D_{i}^{\varepsilon}$ and the production/degradation terms $F_{i}^{\varepsilon}$, with $i=z, s p, a w, a p$, are determined by $Y$-periodic functions $D_{i}(t, y)$ and $F_{i}(t, y, \xi)$, i.e. $D_{i}^{\varepsilon}(t, x)=D_{i}\left(t, \frac{x}{\varepsilon}\right)$ and $F_{i}^{\varepsilon}(t, x, \xi)=$ $F_{i}\left(t, \frac{x}{\varepsilon}, \xi\right)$ for $\xi \in \mathbb{R}$ and $(t, x) \in(0, T) \times \Omega_{i}^{\varepsilon}$. The symplast and apoplast are coupled by diffusion fluxes and via protein mediated transport of solute through the cell membrane described by the following boundary conditions based on Eqs. (2.20a) and (2.20b)

$$
\begin{array}{rlrl}
\left(D_{z}^{\varepsilon} \nabla c_{z}^{\varepsilon}-H_{M}\left(\boldsymbol{v}_{z}^{\varepsilon}\right) c_{z}^{\varepsilon}\right) \cdot \boldsymbol{n}= & \left(D_{s p}^{\varepsilon} \nabla c_{s p}^{\varepsilon}-H_{M}\left(\boldsymbol{v}_{s p}^{\varepsilon}\right) c_{s p}^{\varepsilon}\right) \cdot \boldsymbol{n} & \\
& +\varepsilon \beta_{a}^{\varepsilon}(t, x) \vartheta_{b, a}^{\varepsilon}-\varepsilon \alpha_{s}^{\varepsilon}(t, x) c_{z}^{\varepsilon} \vartheta_{f, s}^{\varepsilon} & & \text { on } \Gamma_{a s}^{\varepsilon} \times(0, T), \\
\left(D_{z}^{\varepsilon} \nabla c_{z}^{\varepsilon}-H_{M}\left(\boldsymbol{v}_{z}^{\varepsilon}\right) c_{z}^{\varepsilon}\right) \cdot \boldsymbol{n}= & \varepsilon \beta_{a}^{\varepsilon}(t, x) \vartheta_{b, a}^{\varepsilon}-\varepsilon \alpha_{s}^{\varepsilon}(t, x) c_{z}^{\varepsilon} \vartheta_{f, s}^{\varepsilon} & & \text { on } \Gamma_{z}^{\varepsilon} \times(0, T), \\
\left(D_{a w}^{\varepsilon} \nabla c_{a w}^{\varepsilon}-H_{M}\left(\boldsymbol{v}_{a w}^{\varepsilon}\right) c_{a w}^{\varepsilon}\right) \cdot \boldsymbol{n}= & \varepsilon \alpha_{a}^{\varepsilon}(t, x) c_{a w}^{\varepsilon} \vartheta_{f, a}^{\varepsilon}-\varepsilon \beta_{s}^{\varepsilon}(t, x) \vartheta_{b, s}^{\varepsilon} & & \text { on } \Gamma_{z}^{\varepsilon} \times(0, T), \\
\left(D_{a p}^{\varepsilon} \nabla c_{a p}^{\varepsilon}-H_{M}\left(\boldsymbol{v}_{a p}^{\varepsilon}\right) c_{a p}^{\varepsilon}\right) \cdot \boldsymbol{n}= & \varepsilon \alpha_{a}^{\varepsilon}(t, x) c_{a p}^{\varepsilon} \vartheta_{f, a}^{\varepsilon}-\varepsilon \beta_{s}^{\varepsilon}(t, x) \vartheta_{b, s}^{\varepsilon} & & \text { on } \Gamma_{a s}^{\varepsilon} \times(0, T),
\end{array}
$$

where $\boldsymbol{n}$ denotes the outer normal vector to $\partial \Omega_{z}^{\varepsilon}$ and the protein mediated flux $\boldsymbol{a}_{2, \mathrm{I}}$ was expressed by relation (2.12) with $\alpha_{l}^{\varepsilon}$ and $\beta_{l}^{\varepsilon}$, for $l=a, s$, are related to the reaction rate coefficients $k_{i}$, for $i=1,2,3$. We define the apoplastic and symplastic concentrations $c_{a}^{\varepsilon}$ and $c_{s}^{\varepsilon}$, respectively, as follows

$$
c_{a}^{\varepsilon}(t, x)=\left\{\begin{array}{ll}
c_{a w}^{\varepsilon}(t, x) & \text { in } \Omega_{a w}^{\varepsilon} \times(0, T), \\
c_{a p}^{\varepsilon}(t, x) & \text { in } \Omega_{a s}^{\varepsilon} \times(0, T),
\end{array} \quad \text { and } \quad c_{s}^{\varepsilon}(t, x)=\left\{\begin{array}{cc}
c_{z}^{\varepsilon}(t, x) & \text { in } \Omega_{z}^{\varepsilon} \times(0, T), \\
c_{s p}^{\varepsilon}(t, x) & \text { in } \Omega_{a s}^{\varepsilon} \times(0, T) .
\end{array}\right.\right.
$$

The dynamics of the transporter concentrations are modelled by following ordinary differential equations

$$
\begin{aligned}
\partial_{t} \vartheta_{f, l}^{\varepsilon} & =R_{l}^{\varepsilon}\left(t, x, \vartheta_{f, l}^{\varepsilon}\right)-\alpha_{l}^{\varepsilon}(t, x) c_{l}^{\varepsilon} \vartheta_{f, l}^{\varepsilon}+\beta_{l}^{\varepsilon}(t, x) \vartheta_{b, l}^{\varepsilon}-\gamma_{f, l}^{\varepsilon}(t, x) \vartheta_{f, l}^{\varepsilon} & & \text { on } \Gamma_{z s}^{\varepsilon} \times(0, T), \\
\partial_{t} \vartheta_{b, l}^{\varepsilon} & =r \alpha_{l}^{\varepsilon}(t, x) c_{l}^{\varepsilon} \vartheta_{f, l}^{\varepsilon}-\beta_{l}^{\varepsilon}(t, x) \vartheta_{b, l}^{\varepsilon}-\gamma_{b, l}^{\varepsilon}(t, x) \vartheta_{b, l}^{\varepsilon} & & \text { on } \Gamma_{z s}^{\varepsilon} \times(0, T),
\end{aligned}
$$

with $l=a, s$, and $\gamma_{j, l}^{\varepsilon}$, for $j=f, b$ are rates of decay of corresponding transporter concentration and $R_{l}^{\varepsilon}$ are production/degradation terms representing the genetic regulation of the transporters. The 
main difference to Eq. (2.11) is the inclusion of the decay terms, which model a possible instability of the transporting proteins. The coefficients and $R_{l}^{\varepsilon}$ are given by $Y$-periodic functions $R_{l}, \alpha_{l}, \beta_{l}$ and $\gamma_{j, l}$, defined on $\Gamma_{z s} \times(0, T)$, i.e. $R_{l}^{\varepsilon}(t, x, \xi)=R_{l}\left(t, \frac{x}{\varepsilon}, \xi\right)$ and $\alpha_{l}^{\varepsilon}(t, x)=\alpha_{l}\left(t, \frac{x}{\varepsilon}\right), \beta_{l}^{\varepsilon}(t, x)=\beta_{l}\left(t, \frac{x}{\varepsilon}\right)$, $\gamma_{j, l}^{\varepsilon}(t, x)=\gamma_{j, l}\left(t, \frac{x}{\varepsilon}\right)$, for $\xi \in \mathbb{R}$ and $(x, t) \in \Gamma_{z s}^{\varepsilon} \times(0, T)$, with $l=a, s$ and $j=f, b$.

In contiguous apoplast and symplast in plasmodesmata populated regions, we pose continuity and zero-flux boundary conditions - based on Eqs. (2.20a) and (2.20b) taking into consideration that no transporters are present $\left(\boldsymbol{a}_{2, \mathrm{I}}=0\right.$ and $\left.\left[\boldsymbol{a}_{2}\right]=0\right)$ - and set the concentration to be continuous

$$
\begin{aligned}
\left(D_{a w}^{\varepsilon} \nabla c_{a w}^{\varepsilon}-H_{M}\left(\boldsymbol{v}_{a w}^{\varepsilon}\right) c_{a w}^{\varepsilon}\right) \cdot \boldsymbol{n}_{a w} & =\left(D_{a p}^{\varepsilon} \nabla c_{a p}^{\varepsilon}-H_{M}\left(\boldsymbol{v}_{a p}^{\varepsilon}\right) c_{a p}^{\varepsilon}\right) \cdot \boldsymbol{n}_{a w} & & \text { on } \Gamma_{a w}^{\varepsilon} \times(0, T), \\
\left(D_{s p}^{\varepsilon} \nabla c_{s p}^{\varepsilon}-H_{M}\left(\boldsymbol{v}_{s p}^{\varepsilon}\right) c_{s p}^{\varepsilon}\right) \cdot \boldsymbol{n}_{a w} & =0 & & \text { on } \Gamma_{a w}^{\varepsilon} \times(0, T), \\
c_{a w}^{\varepsilon} & =c_{a p}^{\varepsilon} & & \text { on } \Gamma_{a w}^{\varepsilon} \times(0, T), \\
c_{z}^{\varepsilon} & =c_{s p}^{\varepsilon} & & \text { on } \Gamma_{a s}^{\varepsilon} \times(0, T),
\end{aligned}
$$

where $\boldsymbol{n}_{a w}$ is the outer normal vector to $\partial \Omega_{a w}^{\varepsilon}$. Initial conditions for solute and transporter concentrations are posed as follows

$$
\begin{aligned}
c_{l}^{\varepsilon}(0, x) & =c_{l}^{0}(x) & & \text { in } \Omega_{l}^{\varepsilon}, \\
\vartheta_{j, l}^{\varepsilon}(0, x) & =\vartheta_{j, l}^{\varepsilon, 0}(x) & & \text { on } \Gamma_{z s}^{\varepsilon},
\end{aligned}
$$

where $j=f, b$ and $l=a, s$. The initial conditions for transporter concentrations are defined as $\vartheta_{j, l}^{\varepsilon, 0}(x)=$ $\vartheta_{j, l}^{0}(x, x / \varepsilon)$ for $x \in \Gamma_{z s}^{\varepsilon}$, where $\vartheta_{j, l}^{0}(x, y)=\vartheta_{1 j, l}^{0}(x) \vartheta_{2 j, l}^{0}(y)$ for $(x, y) \in \Omega \times \Gamma_{z s}$, and $\vartheta_{2 j, l}^{0}$ are $Y$-periodic, with $j=f, b$ and $l=a, s$.

Following conditions, based on (2.20c), (2.20d) and (2.20e), are considered for the velocity of water on internal boundaries

$$
\begin{aligned}
& \left.\begin{array}{rl}
\kappa_{1}\left(-2 \varepsilon^{2} \eta\left(\mathrm{S}_{z}^{\varepsilon} \boldsymbol{n}\right) \cdot \boldsymbol{n}+p_{z}^{\varepsilon}-p_{a w}^{\varepsilon}\right) & =\varepsilon \delta_{1}\left(c_{z}^{\varepsilon}-c_{a w}^{\varepsilon}\right)+\varepsilon \boldsymbol{v}_{a w}^{\varepsilon} \cdot \boldsymbol{n} \\
\boldsymbol{v}_{z}^{\varepsilon} \cdot \boldsymbol{n}=\boldsymbol{v}_{a w}^{\varepsilon} \cdot \boldsymbol{n}, \quad \boldsymbol{v}_{z}^{\varepsilon} \times \boldsymbol{n} & =0
\end{array}\right\} \quad \text { on } \Gamma_{z}^{\varepsilon} \times(0, T) \\
& \left.\begin{array}{c}
-2 \varepsilon^{2} \eta\left(\mathrm{S} \boldsymbol{v}_{z}^{\varepsilon} \boldsymbol{n}\right) \cdot \boldsymbol{n}+p_{z}^{\varepsilon}=p_{s p}^{\varepsilon} \\
\kappa_{2}\left(-2 \varepsilon^{2} \eta\left(\mathrm{S} \boldsymbol{v}_{z}^{\varepsilon} \boldsymbol{n}\right) \cdot \boldsymbol{n}+p_{z}^{\varepsilon}-p_{a p}^{\varepsilon}\right)=\varepsilon \delta_{2}\left(c_{z}^{\varepsilon}-c_{a p}^{\varepsilon}\right)+\varepsilon \boldsymbol{v}_{a p}^{\varepsilon} \cdot \boldsymbol{n} \\
\boldsymbol{v}_{z}^{\varepsilon} \cdot \boldsymbol{n}=\boldsymbol{v}_{s p}^{\varepsilon} \cdot \boldsymbol{n}+\boldsymbol{v}_{a p}^{\varepsilon} \cdot \boldsymbol{n}, \quad \boldsymbol{v}_{z}^{\varepsilon} \times \boldsymbol{n}=0
\end{array}\right\} \quad \text { on } \Gamma_{a s}^{\varepsilon} \times(0, T) \\
& p_{a w}^{\varepsilon}=p_{a p}^{\varepsilon}, \quad \boldsymbol{v}_{a w}^{\varepsilon} \cdot \boldsymbol{n}_{a w}=\boldsymbol{v}_{a p}^{\varepsilon} \cdot \boldsymbol{n}_{a w}, \quad \boldsymbol{v}_{s p}^{\varepsilon} \cdot \boldsymbol{n}_{a w}=0 \quad \text { on } \Gamma_{a w}^{\varepsilon} \times(0, T) .
\end{aligned}
$$

On the external boundary $\partial \Omega$ we assume zero-flux boundary conditions for concentrations

$$
\begin{array}{rlrl}
\left(H_{M}\left(\boldsymbol{v}_{a w}^{\varepsilon}\right) c_{a w}^{\varepsilon}-D_{a w}^{\varepsilon} \nabla c_{a w}^{\varepsilon}\right) \cdot \boldsymbol{n}_{e x} & =0 & & \text { on }\left(\partial \Omega \backslash \partial \Omega_{a s}^{\varepsilon}\right) \times(0, T), \\
\left(H_{M}\left(\boldsymbol{v}_{i}^{\varepsilon}\right) c_{i}^{\varepsilon}-D_{i}^{\varepsilon} \nabla c_{i}^{\varepsilon}\right) \cdot \boldsymbol{n}_{e x}=0 & & \text { on }\left(\partial \Omega \cap \partial \Omega_{a s}^{\varepsilon}\right) \times(0, T),
\end{array}
$$

where $i=a p, s p$ and $\boldsymbol{n}_{e x}$ is the outer normal vector to $\partial \Omega$. We further prescribe symplastic and apoplastic normal velocities

$$
\begin{array}{ll}
\boldsymbol{v}_{a w}^{\varepsilon} \cdot \boldsymbol{n}_{e x}=v_{D} & \text { on }\left(\partial \Omega_{a w}^{\varepsilon} \cap \partial \Omega\right) \times(0, T), \\
\boldsymbol{v}_{a p}^{\varepsilon} \cdot \boldsymbol{n}_{e x}=v_{D} & \text { on }\left(\partial \Omega_{a s}^{\varepsilon} \cap \partial \Omega\right) \times(0, T), \\
\boldsymbol{v}_{s p}^{\varepsilon} \cdot \boldsymbol{n}_{e x}=0 & \text { on }\left(\partial \Omega_{a s}^{\varepsilon} \cap \partial \Omega\right) \times(0, T) .
\end{array}
$$

To simplify notations, we define similar to (3.4) apoplastic and symplastic flow variables

$$
\begin{aligned}
& v_{a}^{\varepsilon}(t, x)=\left\{\begin{array}{ll}
v_{a w}^{\varepsilon}(t, x) & \text { in } \Omega_{a w}^{\varepsilon} \times(0, T), \\
v_{a p}^{\varepsilon}(t, x) & \text { in } \Omega_{a s}^{\varepsilon} \times(0, T),
\end{array} \quad \text { and } \quad v_{s}^{\varepsilon}(t, x)= \begin{cases}v_{z}^{\varepsilon}(t, x) & \text { in } \Omega_{z}^{\varepsilon} \times(0, T), \\
v_{s p}^{\varepsilon}(t, x) & \text { in } \Omega_{a s}^{\varepsilon} \times(0, T),\end{cases} \right. \\
& p_{a}^{\varepsilon}(t, x)=\left\{\begin{array}{ll}
p_{a w}^{\varepsilon}(t, x) & \text { in } \Omega_{a w}^{\varepsilon} \times(0, T), \\
p_{a p}^{\varepsilon}(t, x) & \text { in } \Omega_{a s}^{\varepsilon} \times(0, T),
\end{array} \quad \text { and } \quad p_{s}^{\varepsilon}(t, x)= \begin{cases}p_{z}^{\varepsilon}(t, x) & \text { in } \Omega_{z}^{\varepsilon} \times(0, T), \\
p_{s p}^{\varepsilon}(t, x) & \text { in } \Omega_{a s}^{\varepsilon} \times(0, T) .\end{cases} \right.
\end{aligned}
$$


The corresponding diffusion coefficients and permeability tensors are given by

$$
\begin{aligned}
D_{a}(t, y) & =\left\{\begin{array}{l}
D_{a w}(t, y) \text { in } Y_{a w} \times(0, T), \\
D_{a p}(t, y) \text { in } Y_{a s} \times(0, T),
\end{array} \quad \text { and } \quad D_{s}(t, y)=\left\{\begin{array}{l}
D_{z}(t, y) \text { in } Y_{z} \times(0, T), \\
D_{s p}(t, y) \text { in } Y_{a s} \times(0, T),
\end{array}\right.\right. \\
K_{a}(y) & =\left\{\begin{array}{l}
K_{a w}(y) \text { in } Y_{a w} \times(0, T), \\
K_{a p}(y) \text { in } Y_{a s} \times(0, T) .
\end{array}\right.
\end{aligned}
$$

The production/degradation terms are defined by

$$
F_{a}^{\varepsilon}\left(t, x, c_{a}^{\varepsilon}\right)=\left\{\begin{array}{l}
F_{a w}^{\varepsilon}\left(t, x, c_{a w}^{\varepsilon}\right) \text { in } \Omega_{a w}^{\varepsilon} \times(0, T), \\
F_{a p}^{\varepsilon}\left(t, x, c_{a p}^{\varepsilon}\right) \text { in } \Omega_{a s}^{\varepsilon} \times(0, T),
\end{array} \quad \text { and } \quad F_{s}^{\varepsilon}\left(t, x, c_{s}^{\varepsilon}\right)=\left\{\begin{array}{l}
F_{z}^{\varepsilon}\left(t, x, c_{z}^{\varepsilon}\right) \text { in } \Omega_{z}^{\varepsilon} \times(0, T) \\
F_{s p}^{\varepsilon}\left(t, x, c_{s p}^{\varepsilon}\right) \text { in } \Omega_{a s}^{\varepsilon} \times(0, T)
\end{array}\right.\right.
$$

We assume that $\delta_{i} \neq 0$ and $\kappa_{i} \neq 0$, for $i=1,2$, and define $\kappa^{\varepsilon}(x)=\kappa(x / \varepsilon), \delta^{\varepsilon}(x)=\delta(x / \varepsilon)$ for $x \in \Gamma_{z s}^{\varepsilon}$, where $\kappa, \delta$ are $Y$-periodic functions given by $\kappa(y)=\kappa_{1}^{-1}$ on $\Gamma_{z}, \kappa(y)=\kappa_{2}^{-1}$ on $\Gamma_{a s}$ and $\delta(y)=\delta_{1} \kappa_{1}^{-1}$ on $\Gamma_{z}, \delta(y)=\delta_{2} \kappa_{2}^{-1}$ on $\Gamma_{a s}$.

Assumption 1. - Tensors $K_{a} \in L^{\infty}\left(Y_{a}\right)^{3 \times 3}, K_{s p} \in L^{\infty}\left(Y_{a s}\right)^{3 \times 3}$ are symmetric and uniformly elliptic, i.e. $\left(K_{l}(y) \xi, \xi\right) \geq k_{l}|\xi|^{2}$ for $k_{l}>0, \xi \in \mathbb{R}^{3}$, a.a. $y \in Y_{l}$, where $l=a, s p$ and $Y_{s p}:=Y_{a s}$.

- Diffusion coefficients $D_{l}, \partial_{t} D_{l} \in L^{\infty}\left((0, T) \times Y_{l}\right)^{3 \times 3}$ are symmetric and uniformly elliptic, i.e. $\left(D_{l}(t, y) \xi, \xi\right) \geq d_{l}|\xi|^{2}$ for $d_{l}>0, \xi \in \mathbb{R}^{3}$, a.a. $(t, y) \in(0, T) \times Y_{l}$, where $l=a, s$.

- Production/degradation $F_{l}:(0, T) \times Y_{l} \times \mathbb{R} \rightarrow \mathbb{R}$ is differentiable in $t$, measurable in $y, F_{l}$ and $\partial_{t} F_{l}$ are sublinear in $\xi, F_{l}$ is Lipschitz continuous in $\xi$ uniformly in $(t, y)$, and $F_{l}\left(t, y, \xi_{-}\right) \xi_{-} \leq C\left|\xi_{-}\right|^{2}$ for $(t, y) \in(0, T) \times Y_{l}$, where $\xi_{-}=\min \{\xi, 0\}$ and $l=a, s$.

- Functions $R_{l}(\cdot, \cdot, \xi) \in C\left([0, T] ; L^{\infty}\left(\Gamma_{z s}\right)\right)$ for all $\xi \in \mathbb{R}$ are uniformly Lipschitz continuous in $\xi$, and nonnegative for nonnegative $\xi$ and $(x, t) \in \Gamma_{z s} \times[0, T]$, with $l=a, s$.

- Coefficients $\alpha_{l}, \beta_{l}, \gamma_{j, l} \in C\left([0, T] ; L^{\infty}\left(\Gamma_{z s}\right)\right)$ are nonnegative and $\partial_{t} \alpha_{l}, \partial_{t} \beta_{l} \in L^{\infty}\left((0, T) \times \Gamma_{z s}\right)$, where $j=f, b$ and $l=a, s$.

- Initial conditions $c_{l}^{0} \in H^{2}(\Omega)$ and $\vartheta_{1 j, l}^{0} \in L^{\infty}(\Omega), \vartheta_{2 j, l}^{0} \in L^{\infty}\left(\Gamma_{z s}\right)$ are nonnegative, $l=a, s, j=f, b$.

- Boundary condition $v_{D} \in H^{1 / 2}(\partial \Omega)$ is given by $v_{D}=V_{D} \cdot \boldsymbol{n}_{e x}$ on $\partial \Omega$, where $V_{D} \in H^{1}(\Omega)$ and $\operatorname{div} V_{D}=0$ in $\Omega$.

By Lemma 4 in [38], there exists a restriction operator $R_{Y_{a}} \in \mathcal{L}\left(H^{1}(Y), H_{\Gamma_{z s}}^{1}\left(Y_{a}\right)\right)$ with the properties

$$
\begin{cases}R_{Y_{a}} \psi=\psi & \text { in a neighborhood of } \partial Y \\ \psi=0 \text { on } \Gamma_{z s} & \Longrightarrow R_{Y_{a}} \psi=\psi \\ \operatorname{div} \psi=0 & \Longrightarrow \operatorname{div} R_{Y_{a}} \psi=0\end{cases}
$$

Here $H_{\Gamma_{z s}}^{1}\left(Y_{a}\right)=\left\{\psi \in H^{1}\left(Y_{a}\right): \psi=0\right.$ on $\left.\Gamma_{z s}\right\}$. For $\psi \in H^{1}(\Omega)$ we define $\psi_{j}^{\varepsilon}(y)=\psi(\varepsilon y)$ for $y \in$ $Y^{j}=\left(Y+k^{j}\right)$, with $j=1, \ldots, J$, where $J \in \mathbb{N}$ such that $\Omega=\cup_{j=1}^{J} \varepsilon Y^{j}$, and $k^{j} \in \mathbb{Z}^{3}$. Then the operator defined as $\tilde{R}_{\Omega_{a}^{\varepsilon}}^{\varepsilon} \psi(x)=\left(R_{Y_{a}} \psi_{j}^{\varepsilon}\right)(x / \varepsilon)$ for $x \in \varepsilon Y_{a}^{j}$, with $j=1, \ldots, J$, has the properties that $\tilde{R}_{\Omega_{a}^{\varepsilon}} \in \mathcal{L}\left(H^{1}(\Omega), H_{\Gamma_{z s}^{\varepsilon}}^{1}\left(\Omega_{a}^{\varepsilon}\right)\right)$ and

$$
\begin{cases}\psi=0 \text { on } \Gamma_{z s}^{\varepsilon} & \Longrightarrow \tilde{R}_{\Omega_{a}^{\varepsilon}}^{\varepsilon} \psi=\psi, \\ \operatorname{div} \psi=0 & \Longrightarrow \operatorname{div} \tilde{R}_{\Omega_{a}^{\varepsilon}}^{\varepsilon} \psi=0, \\ \left\|\tilde{R}_{\Omega_{a}^{\varepsilon}}^{\varepsilon} \psi\right\|_{L^{2}\left(\Omega_{a}^{\varepsilon}\right)} \leq C\left(\|\psi\|_{L^{2}(\Omega)}+\varepsilon\|\nabla \psi\|_{L^{2}(\Omega)}\right), \\ \varepsilon\left\|\nabla \tilde{R}_{\Omega_{a}^{\varepsilon}}^{\varepsilon} \psi\right\|_{L^{2}\left(\Omega_{a}^{\varepsilon}\right)} \leq C\left(\|\psi\|_{L^{2}(\Omega)}+\varepsilon\|\nabla \psi\|_{L^{2}(\Omega)}\right),\end{cases}
$$

where $H_{\Gamma_{z s}^{\varepsilon}}^{1}\left(\Omega_{a}^{\varepsilon}\right)=\left\{\psi \in H^{1}\left(\Omega_{a}^{\varepsilon}\right): \psi=0\right.$ on $\left.\Gamma_{z s}^{\varepsilon}\right\}$.

We define $V_{D}^{\varepsilon}=\tilde{R}_{\Omega_{a}^{\varepsilon}} V_{D}$ and, using the assumptions on $V_{D}$, obtain that $\operatorname{div} V_{D}^{\varepsilon}=0$ in $\Omega_{a}^{\varepsilon}$ and $\left\|V_{D}^{\varepsilon}\right\|_{L^{2}\left(\Omega_{a}^{\varepsilon}\right)} \leq C\left\|V_{D}\right\|_{H^{1}(\Omega)}$. 
For a $\sigma>0$, a Lipschitz domain $\Sigma$, and for $\psi, \varphi \in L^{2}((0, \sigma) \times \Sigma)$ we shall denote

$$
\langle\psi, \varphi\rangle_{\Sigma}=\int_{\Sigma} \psi \varphi d x \quad \text { and } \quad\langle\psi, \varphi\rangle_{\Sigma, \sigma}=\int_{0}^{\sigma} \int_{\Sigma} \psi \varphi d x d t .
$$

For a bounded Lipschitz domain $\Sigma$ we shall use the notion of the space

$$
H(\operatorname{div}, \Sigma)=\left\{\boldsymbol{v} \in L^{2}(\Sigma) \text { such that } \operatorname{div} \boldsymbol{v} \in L^{2}(\Sigma)\right\},
$$

provided with the norm

$$
\|\boldsymbol{v}\|_{H(\operatorname{div}, \Sigma)}^{2}=\|\boldsymbol{v}\|_{L^{2}(\Sigma)}^{2}+\|\operatorname{div} \boldsymbol{v}\|_{L^{2}(\Sigma)}^{2} .
$$

We introduce also

$$
\begin{aligned}
V_{a s}^{\varepsilon} & =\left\{v \in H\left(\operatorname{div}, \Omega_{a s}^{\varepsilon}\right): v \cdot \boldsymbol{n}=0 \text { on } \partial \Omega_{a s}^{\varepsilon} \cap\left(\partial \Omega_{a w}^{\varepsilon} \cup \partial \Omega\right)\right\} \\
V_{a}^{\varepsilon} & =\left\{v \in H\left(\operatorname{div}, \Omega_{a}^{\varepsilon}\right): v \cdot \boldsymbol{n} \in L^{2}\left(\Gamma_{z s}^{\varepsilon}\right), v \cdot \boldsymbol{n}=0 \text { on } \partial \Omega\right\} \\
V_{z}^{\varepsilon} & =\left\{v \in H^{1}\left(\Omega_{z}^{\varepsilon}\right): v \times \boldsymbol{n}=0 \text { on } \Gamma_{z s}^{\varepsilon}\right\}
\end{aligned}
$$

For $\phi \in V_{a s}^{\varepsilon}$, since $\phi \in H\left(\operatorname{div}, \Omega_{a s}^{\varepsilon}\right)$ we have $\phi \cdot \boldsymbol{n} \in H^{-1 / 2}\left(\partial \Omega_{a s}^{\varepsilon}\right)$ and together with $\phi \cdot \boldsymbol{n}=0$ on $\partial \Omega_{a s}^{\varepsilon} \backslash \Gamma_{a s}^{\varepsilon}$ obtain $\phi \cdot \boldsymbol{n} \in H^{-1 / 2}\left(\Gamma_{a s}^{\varepsilon}\right),[15]$. Considering the geometrical structure of $\Omega_{a s}^{\varepsilon}$ and the fact that $\phi \cdot \boldsymbol{n}=0$ on $\Gamma_{a w}^{\varepsilon}$, we can extend $\phi$ by zero from $\Omega_{a s}^{\varepsilon}$ to $\Omega_{a}^{\varepsilon}$ with $\operatorname{div} \phi \in L^{2}\left(\Omega_{a}^{\varepsilon}\right)$ and shall use the same notation for the extension as for the original function.

We denote the spaces

$$
\begin{aligned}
& \mathcal{V}^{\varepsilon}=\left\{\left(v_{1}, v_{2}, v_{3}\right) \in V_{z}^{\varepsilon} \times V_{a}^{\varepsilon} \times V_{a s}^{\varepsilon}:\left\langle\left(v_{1}-v_{2}\right) \cdot \boldsymbol{n}, \phi\right\rangle_{\Gamma_{z}^{\varepsilon}}=0 \text { for } \phi \in L^{2}\left(\Gamma_{z}^{\varepsilon}\right),\right. \\
& \left.\quad\left\langle\left(v_{1}-v_{2}-v_{3}\right) \cdot \boldsymbol{n}, \psi\right\rangle_{H^{-1 / 2}\left(\Gamma_{a s}^{\varepsilon}\right), H^{1 / 2}\left(\Gamma_{a s}^{\varepsilon}\right)}=0 \text { for } \psi \in H^{1 / 2}\left(\Gamma_{a s}^{\varepsilon}\right)\right\}, \\
& \mathcal{P}^{\varepsilon}=\left\{\left(p_{1}, p_{2}, p_{3}\right) \in L^{2}\left(\Omega_{z}^{\varepsilon}\right) \times L^{2}\left(\Omega_{a}^{\varepsilon}\right) \times L^{2}\left(\Omega_{a s}^{\varepsilon}\right):\left\langle p_{1}, 1\right\rangle_{\Omega_{z}^{\varepsilon}}+\left\langle p_{2}, 1\right\rangle_{\Omega_{a}^{\varepsilon}}+\left\langle p_{3}, 1\right\rangle_{\Omega_{a s}^{\varepsilon}}=0\right\},
\end{aligned}
$$

with the norms

$$
\begin{aligned}
& \|v\|_{\mathcal{V}^{\varepsilon}}=\left\|v_{1}\right\|_{H\left(\operatorname{div}, \Omega_{z}^{\varepsilon}\right)}+\varepsilon\left\|\nabla v_{1}\right\|_{L^{2}\left(\Omega_{z}^{\varepsilon}\right)}+\left\|v_{2}\right\|_{H\left(\operatorname{div}, \Omega_{a}^{\varepsilon}\right)}+\varepsilon^{1 / 2}\left\|v_{2} \cdot \boldsymbol{n}\right\|_{L^{2}\left(\Gamma_{z s}^{\varepsilon}\right)}+\left\|v_{3}\right\|_{H\left(\operatorname{div}, \Omega_{a s}^{\varepsilon}\right)}, \\
& \|p\|_{\mathcal{P}^{\varepsilon}}=\left\|p_{1}\right\|_{L^{2}\left(\Omega_{z}^{\varepsilon}\right)}+\left\|p_{2}\right\|_{L^{2}\left(\Omega_{a}^{\varepsilon}\right)}+\left\|p_{3}\right\|_{L^{2}\left(\Omega_{a s}^{\varepsilon}\right)} .
\end{aligned}
$$

Notice that for $\psi \in \mathcal{V}^{\varepsilon}$ due to the assumptions on the normal components at the boundaries we have $\operatorname{div} \varphi \in L^{2}(\Omega)$, where $\varphi=\psi_{1}$ in $\Omega_{z}^{\varepsilon}, \varphi=\psi_{2}$ in $\Omega_{a w}^{\varepsilon}$ and $\varphi=\psi_{2}+\psi_{3}$ in $\Omega_{a s}^{\varepsilon}$. We shall consider also $L^{2}\left(0, T ; \mathcal{V}^{\varepsilon}\right)$ and $L^{2}\left(0, T ; \mathcal{P}^{\varepsilon}\right)$ with the norms

$$
\begin{gathered}
\|v\|_{L^{2}\left(0, T ; \mathcal{V}^{\varepsilon}\right)}=\left\|v_{1}\right\|_{L^{2}\left(0, T ; H\left(\operatorname{div}, \Omega_{z}^{\varepsilon}\right)\right)}+\varepsilon\left\|\nabla v_{1}\right\|_{L^{2}\left((0, T) \times \Omega_{z}^{\varepsilon}\right)}+\left\|v_{2}\right\|_{L^{2}\left(0, T ; H\left(\operatorname{div}, \Omega_{a}^{\varepsilon}\right)\right)} \\
+\varepsilon^{1 / 2}\left\|v_{2} \cdot \boldsymbol{n}\right\|_{L^{2}\left((0, T) \times \Gamma_{z s}^{\varepsilon}\right)}+\left\|v_{3}\right\|_{L^{2}\left(0, T ; H\left(\operatorname{div}, \Omega_{a s}^{\varepsilon}\right)\right)} \\
\|p\|_{L^{2}\left(0, T ; \mathcal{P}^{\varepsilon}\right)}=\left\|p_{1}\right\|_{L^{2}\left((0, T) \times \Omega_{z}^{\varepsilon}\right)}+\left\|p_{2}\right\|_{L^{2}\left((0, T) \times \Omega_{a}^{\varepsilon}\right)}+\left\|p_{3}\right\|_{L^{2}\left((0, T) \times \Omega_{a s}^{\varepsilon}\right)} .
\end{gathered}
$$

The corresponding divergence-free space is denoted by

$$
\mathcal{V}_{d}^{\varepsilon}=\left\{\left(v_{1}, v_{2}, v_{3}\right) \in \mathcal{V}^{\varepsilon}: \operatorname{div} v_{1}=0 \text { in } \Omega_{z}^{\varepsilon}, \operatorname{div} v_{2}=0 \text { in } \Omega_{a}^{\varepsilon}, \operatorname{div} v_{3}=0 \text { in } \Omega_{a s}^{\varepsilon}\right\} .
$$

\section{Well-posedness and a priori estimates}

We start with a weak formulation of the microscopic model (3.1)-(3.10). 
Definition 4.1. Weak solution of (3.1)-(3.10) are velocity field $\left(\boldsymbol{v}_{z}^{\varepsilon}, \boldsymbol{v}_{a}^{\varepsilon}-V_{D}^{\varepsilon}, \boldsymbol{v}_{s p}^{\varepsilon}\right) \in L^{2}\left(0, T ; \mathcal{V}^{\varepsilon}\right)$, and pressure $p^{\varepsilon}=\left(p_{z}^{\varepsilon}, p_{a}^{\varepsilon}, p_{s p}^{\varepsilon}\right) \in L^{2}\left(0, T ; \mathcal{P}^{\varepsilon}\right)$, satisfying equations (3.1) with boundary conditions (3.8) and (3.10) in the variational formulation

$$
\begin{array}{r}
\varepsilon^{2}\left\langle 2 \eta \mathrm{S} \boldsymbol{v}_{z}^{\varepsilon}, \mathrm{S} \psi_{1}\right\rangle_{\Omega_{z}^{\varepsilon}, T}-\left\langle p_{z}^{\varepsilon}, \operatorname{div} \psi_{1}\right\rangle_{\Omega_{z}^{\varepsilon}, T}+\left\langle K_{a, \varepsilon}^{-1}(x) \boldsymbol{v}_{a}^{\varepsilon}, \psi_{2}\right\rangle_{\Omega_{a}^{\varepsilon}, T}-\left\langle p_{a}^{\varepsilon}, \operatorname{div} \psi_{2}\right\rangle_{\Omega_{a}^{\varepsilon}, T} \\
+\left\langle K_{s p, \varepsilon}^{-1}(x) \boldsymbol{v}_{s p}^{\varepsilon}, \psi_{3}\right\rangle_{\Omega_{a s}^{\varepsilon}, T}-\left\langle p_{s p}^{\varepsilon}, \operatorname{div} \psi_{3}\right\rangle_{\Omega_{a s}^{\varepsilon}, T}+\varepsilon\left\langle\kappa^{\varepsilon}(x) \boldsymbol{v}_{a}^{\varepsilon} \cdot \boldsymbol{n}+\delta^{\varepsilon}(x)\left(c_{s}^{\varepsilon}-c_{a}^{\varepsilon}\right), \psi_{2} \cdot \boldsymbol{n}\right\rangle_{\Gamma_{z s}^{\varepsilon}, T}=0, \\
\left\langle\operatorname{div} \boldsymbol{v}_{z}^{\varepsilon}, q_{1}\right\rangle_{\Omega_{z}^{\varepsilon}, T}+\left\langle\operatorname{div} \boldsymbol{v}_{a}^{\varepsilon}, q_{2}\right\rangle_{\Omega_{a}^{\varepsilon}, T}+\left\langle\operatorname{div} \boldsymbol{v}_{a s}^{\varepsilon}, q_{3}\right\rangle_{\Omega_{a s}^{\varepsilon}, T}=0,
\end{array}
$$

for $\psi=\left(\psi_{1}, \psi_{2}, \psi_{3}\right) \in L^{2}\left(0, T ; \mathcal{V}^{\varepsilon}\right)$ and $q=\left(q_{1}, q_{2}, q_{3}\right) \in L^{2}\left(0, T ; \mathcal{P}^{\varepsilon}\right)$, and functions $c_{l}^{\varepsilon} \in L^{\infty}\left((0, T) \times \Omega_{l}^{\varepsilon}\right)$, $c_{l}^{\varepsilon} \in L^{2}\left(0, T ; H^{1}\left(\Omega_{l}^{\varepsilon}\right)\right) \cap H^{1}\left(0, T ; L^{2}\left(\Omega_{l}^{\varepsilon}\right)\right)$, for $l=a, s$, satisfying equations (3.2) with boundary conditions (3.3), (3.6), and (3.9) in the weak form

$$
\begin{array}{r}
\left\langle\partial_{t} c_{a}^{\varepsilon}, \varphi_{1}\right\rangle_{\Omega_{a}^{\varepsilon}, T}+\left\langle D_{a}^{\varepsilon}(t, x) \nabla c_{a}^{\varepsilon}-H_{M}\left(\boldsymbol{v}_{a}^{\varepsilon}\right) c_{a}^{\varepsilon}, \nabla \varphi_{1}\right\rangle_{\Omega_{a}^{\varepsilon}, T}=\varepsilon\left\langle\beta_{s}^{\varepsilon}(t, x) \vartheta_{b, s}^{\varepsilon}\right. \\
\left.-\alpha_{a}^{\varepsilon}(t, x) c_{a}^{\varepsilon} \vartheta_{f, a}^{\varepsilon}, \varphi_{1}\right\rangle_{\Gamma_{z s}^{\varepsilon}, T} \\
+\left\langle F_{a}^{\varepsilon}\left(t, x, c_{a}^{\varepsilon}\right), \varphi_{1}\right\rangle_{\Omega_{a}^{\varepsilon}, T}
\end{array}
$$

and

$$
\begin{array}{r}
\left\langle\partial_{t} c_{s}^{\varepsilon}, \varphi_{2}\right\rangle_{\Omega_{s}^{\varepsilon}, T}+\left\langle D_{s}^{\varepsilon}(t, x) \nabla c_{s}^{\varepsilon}-H_{M}\left(\boldsymbol{v}_{s}^{\varepsilon}\right) c_{s}^{\varepsilon}, \nabla \varphi_{2}\right\rangle_{\Omega_{s}^{\varepsilon}, T}=\varepsilon\left\langle\beta_{a}^{\varepsilon}(t, x) \vartheta_{b, a}^{\varepsilon}\right. \\
\left.-\alpha_{s}^{\varepsilon}(t, x) c_{s}^{\varepsilon} \vartheta_{f, s}^{\varepsilon}, \varphi_{2}\right\rangle_{\Gamma_{z s}^{\varepsilon}, T} \\
+\left\langle F_{s}^{\varepsilon}\left(t, x, c_{s}^{\varepsilon}\right), \varphi_{2}\right\rangle_{\Omega_{s}^{\varepsilon}, T}
\end{array}
$$

for all $\varphi_{1} \in L^{2}\left(0, T ; H^{1}\left(\Omega_{a}^{\varepsilon}\right)\right), \varphi_{2} \in L^{2}\left(0, T ; H^{1}\left(\Omega_{s}^{\varepsilon}\right)\right)$, with $c_{l}^{\varepsilon}(0, x)=c_{l}^{0}(x)$ a.e. in $\Omega_{l}^{\varepsilon}$, and transporter concentrations $\vartheta_{j, l}^{\varepsilon} \in W^{1, \infty}\left(0, T ; L^{\infty}\left(\Gamma_{z s}^{\varepsilon}\right)\right)$, with $j=f, b$ and $l=a, s$, satisfying ordinary differential equations (3.5) a.e. on $\Gamma_{z s}^{\varepsilon} \times(0, T)$ together with initial conditions (3.7) a.e. on $\Gamma_{z s}^{\varepsilon}$.

In following we shall use the notation $\bar{V}_{D}^{\varepsilon}=\left(0, V_{D}^{\varepsilon}, 0\right)$ and $\left(\boldsymbol{v}^{\varepsilon}-\bar{V}_{D}^{\varepsilon}\right)=\left(\boldsymbol{v}_{z}^{\varepsilon}, \boldsymbol{v}_{a}^{\varepsilon}-V_{D}^{\varepsilon}, \boldsymbol{v}_{s p}^{\varepsilon}\right)$.

\subsection{Existence and estimates for $\left(v_{s}^{\varepsilon}, p_{s}^{\varepsilon}\right)$ and $\left(v_{a}^{\varepsilon}, p_{a}^{\varepsilon}\right)$}

First we shall prove Korn's type inequality satisfied by functions from the space

$$
\begin{aligned}
\tilde{\mathcal{V}}^{\varepsilon}=\left\{\left(v_{1}, v_{2}, v_{3}\right) \in H^{1}\left(\Omega_{z}^{\varepsilon}\right) \times\right. & V_{a}^{\varepsilon} \times V_{a s}^{\varepsilon}:\left\langle\left(v_{1}-v_{2}\right) \cdot \boldsymbol{n}, \phi\right\rangle_{\Gamma_{z}^{\varepsilon}}=0 \text { for } \phi \in L^{2}\left(\Gamma_{z}^{\varepsilon}\right), \\
& \left.\left\langle\left(v_{1}-v_{2}-v_{3}\right) \cdot \boldsymbol{n}, \psi\right\rangle_{H^{-1 / 2}\left(\Gamma_{a s}^{\varepsilon}\right), H^{1 / 2}\left(\Gamma_{a s}^{\varepsilon}\right)}=0 \text { for } \psi \in H^{1 / 2}\left(\Gamma_{a s}^{\varepsilon}\right)\right\} .
\end{aligned}
$$

Lemma 4.2. For $\boldsymbol{\psi} \in \tilde{\mathcal{V}}^{\varepsilon}$ we have the following Korn's type inequality

$$
\begin{aligned}
\left\|\boldsymbol{\psi}_{1}\right\|_{L^{2}\left(\Omega_{z}^{\varepsilon}\right)} & +\varepsilon\left\|\nabla \boldsymbol{\psi}_{1}\right\|_{L^{2}\left(\Omega_{z}^{\varepsilon}\right)} \leq C\left(\varepsilon\left\|\mathrm{S} \boldsymbol{\psi}_{1}\right\|_{L^{2}\left(\Omega_{z}^{\varepsilon}\right)}+\varepsilon^{1 / 2}\left\|\boldsymbol{\psi}_{1} \times \boldsymbol{n}\right\|_{L^{2}\left(\Gamma_{z s}^{\varepsilon}\right)}+\left\|\boldsymbol{\psi}_{2}\right\|_{L^{2}\left(\Omega_{a}^{\varepsilon}\right)}\right. \\
& \left.+\left\|\boldsymbol{\psi}_{3}\right\|_{L^{2}\left(\Omega_{a s}^{\varepsilon}\right)}+\varepsilon\left\|\operatorname{div} \boldsymbol{\psi}_{1}\right\|_{L^{2}\left(\Omega_{z}^{\varepsilon}\right)}+\varepsilon\left\|\operatorname{div} \boldsymbol{\psi}_{2}\right\|_{L^{2}\left(\Omega_{a}^{\varepsilon}\right)}+\varepsilon\left\|\operatorname{div} \boldsymbol{\psi}_{3}\right\|_{L^{2}\left(\Omega_{a s}^{\varepsilon}\right)}\right) .
\end{aligned}
$$

Proof. The proof follows the same lines as in [4]. First we show the estimate for $\hat{\boldsymbol{\psi}} \in \mathcal{V}(Y)$, where $\mathcal{V}(Y)=\left\{\boldsymbol{\psi}_{1} \in H^{1}\left(Y_{z}\right), \boldsymbol{\psi}_{2} \in \mathcal{V}_{a}(Y), \boldsymbol{\psi}_{3} \in \mathcal{V}_{a s}(Y),\left\langle\left(\boldsymbol{\psi}_{1}-\boldsymbol{\psi}_{2}\right) \cdot \boldsymbol{n}, \phi_{1}\right\rangle_{\Gamma_{z}}=0\right.$ for $\phi_{1} \in L^{2}\left(\Gamma_{z}\right)$, and $\left\langle\left(\boldsymbol{\psi}_{1}-\right.\right.$ $\left.\left.\boldsymbol{\psi}_{2}-\boldsymbol{\psi}_{3}\right) \cdot \boldsymbol{n}, \phi_{2}\right\rangle_{H^{-1 / 2}, H^{1 / 2}}=0$ for $\left.\phi_{2} \in H^{1 / 2}\left(\Gamma_{a s}\right)\right\}$, with $\mathcal{V}_{a}(Y)=\left\{v \in H\left(\operatorname{div}, Y_{a}\right), v \cdot \boldsymbol{n} \in L^{2}\left(\Gamma_{a}\right)\right\}$ and $\mathcal{V}_{a s}(Y)=\left\{v \in H\left(\operatorname{div}, Y_{a s}\right), v \cdot \boldsymbol{n}=0\right.$ on $\left.\Gamma_{a w}\right\}$. Then scaling argument will imply inequality (4.4) for $\psi \in \tilde{\mathcal{V}}^{\varepsilon}$. Suppose it is not true that there exists a constant $\tilde{C}$ such that for $\hat{\boldsymbol{\psi}} \in \mathcal{V}(Y)$

$$
\begin{array}{r}
\left\|\hat{\boldsymbol{\psi}}_{1}\right\|_{L^{2}\left(Y_{z}\right)}+\left\|\nabla \hat{\boldsymbol{\psi}}_{1}\right\|_{L^{2}\left(Y_{z}\right)} \leq \tilde{C}\left(\left\|\mathrm{~S} \hat{\boldsymbol{\psi}}_{1}\right\|_{L^{2}\left(Y_{z}\right)}+\left\|\hat{\boldsymbol{\psi}}_{1} \times \boldsymbol{n}\right\|_{L^{2}\left(\Gamma_{z s}\right)}+\left\|\hat{\boldsymbol{\psi}}_{2}\right\|_{L^{2}\left(Y_{a}\right)}+\left\|\hat{\boldsymbol{\psi}}_{3}\right\|_{L^{2}\left(Y_{a s}\right)}\right. \\
\left.+\left\|\operatorname{div} \hat{\boldsymbol{\psi}}_{1}\right\|_{L^{2}\left(Y_{z}\right)}+\left\|\operatorname{div} \hat{\boldsymbol{\psi}}_{2}\right\|_{L^{2}\left(Y_{a}\right)}+\left\|\operatorname{div} \hat{\boldsymbol{\psi}}_{3}\right\|_{L^{2}\left(Y_{a s}\right)}\right) .
\end{array}
$$

Then there exists a sequence $\left\{\hat{\boldsymbol{\psi}}^{m}\right\} \subset \mathcal{V}(Y)$ such that

$$
\left\|\hat{\boldsymbol{\psi}}_{1}^{m}\right\|_{L^{2}\left(Y_{z}\right)}+\left\|\nabla \hat{\boldsymbol{\psi}}_{1}^{m}\right\|_{L^{2}\left(Y_{z}\right)}=1
$$


and

$$
\begin{aligned}
& \left\|\mathrm{S} \hat{\boldsymbol{\psi}}_{1}^{m}\right\|_{L^{2}\left(Y_{z}\right)}+\left\|\hat{\boldsymbol{\psi}}_{1}^{m} \times \boldsymbol{n}\right\|_{L^{2}\left(\Gamma_{z s}\right)}+\left\|\hat{\boldsymbol{\psi}}_{2}^{m}\right\|_{L^{2}\left(Y_{a}\right)}+\left\|\hat{\boldsymbol{\psi}}_{3}^{m}\right\|_{L^{2}\left(Y_{a s}\right)} \\
& +\left\|\operatorname{div} \hat{\boldsymbol{\psi}}_{1}^{m}\right\|_{L^{2}\left(Y_{z}\right)}+\left\|\operatorname{div} \hat{\boldsymbol{\psi}}_{2}^{m}\right\|_{L^{2}\left(Y_{a}\right)}+\left\|\operatorname{div} \hat{\boldsymbol{\psi}}_{3}^{m}\right\|_{L^{2}\left(Y_{a s}\right)} \leq \frac{1}{m} .
\end{aligned}
$$

The last inequality implies that

$$
\hat{\boldsymbol{\psi}}_{2}^{m} \rightarrow 0 \quad \text { in } H\left(\operatorname{div}, Y_{a}\right) \quad \text { and } \quad \hat{\boldsymbol{\psi}}_{3}^{m} \rightarrow 0 \quad \text { in } H\left(\operatorname{div}, Y_{a s}\right),
$$

whereas, due to (4.6), there exists $\hat{\boldsymbol{\psi}}_{1} \in H^{1}\left(Y_{z}\right)$ such that

$$
\hat{\boldsymbol{\psi}}_{1}^{m} \rightarrow \hat{\boldsymbol{\psi}}_{1} \quad \text { weakly in } H^{1}\left(Y_{z}\right) \text {. }
$$

We shall denote by $\hat{\boldsymbol{\psi}}$ the extension of $\hat{\boldsymbol{\psi}}_{1}$ by zero into $Y$. Since $\hat{\boldsymbol{\psi}}^{m}=\hat{\boldsymbol{\psi}}_{1}^{m} \chi_{Y_{z}}+\hat{\boldsymbol{\psi}}_{2}^{m} \chi_{Y_{a}}+\hat{\boldsymbol{\psi}}_{3}^{m} \chi_{Y_{a s}}$ is bounded in $H(\operatorname{div}, Y)$, it converges weakly and we conclude that

$$
\hat{\boldsymbol{\psi}}^{m} \rightarrow \hat{\boldsymbol{\psi}} \quad \text { weakly in } H(\operatorname{div}, Y) .
$$

Using the estimate for the norm in the space $H^{-1 / 2}\left(\Gamma_{z s}\right)$, i.e.

$$
\left\|\hat{\boldsymbol{\psi}}_{2}^{m} \cdot \boldsymbol{n}\right\|_{H^{-1 / 2}\left(\Gamma_{z s}\right)} \leq C\left\|\hat{\boldsymbol{\psi}}_{2}^{m}\right\|_{H\left(\operatorname{div}, Y_{a}\right)} \rightarrow 0
$$

and the boundary condition $\hat{\boldsymbol{\psi}}_{1}^{m} \cdot \boldsymbol{n}=\hat{\boldsymbol{\psi}}_{2}^{m} \cdot \boldsymbol{n}$ a.e. on $\Gamma_{z}$, we obtain $\hat{\boldsymbol{\psi}}_{1} \cdot \boldsymbol{n}=\hat{\boldsymbol{\psi}}_{2} \cdot \boldsymbol{n}=0$ a.e. on $\Gamma_{z}$. Additionally we have that

$$
\left\|\hat{\boldsymbol{\psi}}_{1}^{m} \times \boldsymbol{n}\right\|_{L^{2}\left(\Gamma_{z s}\right)} \rightarrow 0 \quad \text { as } m \rightarrow \infty, \quad \text { and therefore } \quad \hat{\boldsymbol{\psi}}_{1} \times \boldsymbol{n}=0 \text { a.e. on } \Gamma_{z s} \text {. }
$$

Now the classical Korn inequality in $Y_{z}$ with $\hat{\boldsymbol{\psi}}_{1}=0$ on $\Gamma_{z} \subset \partial Y_{z}$ can be applied and we obtain

$$
\left\|\hat{\boldsymbol{\psi}}_{1}\right\|_{L^{2}\left(Y_{z}\right)}+\left\|\nabla \hat{\boldsymbol{\psi}}_{1}\right\|_{L^{2}\left(Y_{z}\right)} \leq \hat{C}\left\|\mathrm{~S}_{y} \hat{\boldsymbol{\psi}}_{1}\right\|_{L^{2}\left(Y_{z}\right)} .
$$

Considering (4.6) and (4.7), we obtain that the left-hand side in (4.10) is equal to one, whereas the right-hand side is zero. This yields the contradiction to the assumption that there no such constant $\tilde{C}$ for which (4.5) hold true.

Due to the geometrical assumption on $\Omega$ we can write $\Omega=\cup_{j=1}^{J} \varepsilon\left(Y+k^{j}\right)$ with some $J \in \mathbb{N}$ and $k^{j} \in \mathbb{Z}^{3}$. We consider now $\boldsymbol{\psi} \in \mathcal{V}^{\varepsilon}$ and for $y \in Y$ define $\hat{\boldsymbol{\psi}}^{j}(y)=\boldsymbol{\psi}\left(\varepsilon y+\varepsilon k^{j}\right)$, and obtain $\hat{\boldsymbol{\psi}}^{j} \in \mathcal{V}(Y)$. Applying (4.5) for each $Y_{j}=\left(Y+k^{j}\right)$ we obtain estimate for $\hat{\boldsymbol{\psi}}^{j}$

$$
\begin{array}{r}
\left\|\hat{\boldsymbol{\psi}}_{1}^{j}\right\|_{L^{2}\left(Y_{z}^{j}\right)}^{2}+\left\|\nabla \hat{\boldsymbol{\psi}}_{1}^{j}\right\|_{L^{2}\left(Y_{z}^{j}\right)}^{2} \leq \tilde{C}\left(\left\|\mathrm{~S} \hat{\boldsymbol{\psi}}_{1}^{j}\right\|_{L^{2}\left(Y_{z}^{j}\right)}^{2}+\left\|\hat{\boldsymbol{\psi}}_{1}^{j} \times \boldsymbol{n}\right\|_{L^{2}\left(\Gamma_{z s}^{j}\right)}^{2}+\left\|\hat{\boldsymbol{\psi}}_{2}^{j}\right\|_{L^{2}\left(Y_{a}^{j}\right)}^{2}+\left\|\hat{\boldsymbol{\psi}}_{3}^{j}\right\|_{L^{2}\left(Y_{a s}^{j}\right)}^{2}\right. \\
\left.+\left\|\operatorname{div} \hat{\boldsymbol{\psi}}_{1}^{j}\right\|_{L^{2}\left(Y_{z}^{j}\right)}^{2}+\left\|\operatorname{div} \hat{\boldsymbol{\psi}}_{2}^{j}\right\|_{L^{2}\left(Y_{a}^{j}\right)}^{2}+\left\|\operatorname{div} \hat{\boldsymbol{\psi}}_{3}^{j}\right\|_{L^{2}\left(Y_{a s}^{j}\right)}^{2}\right) .
\end{array}
$$

Summation over $j=1, \ldots, J$ and change of variables $x=\varepsilon\left(y+k^{j}\right)$ for $y \in Y$ in (4.11) yield (4.4).

For the proof of existence and uniqueness of $\boldsymbol{v}^{\varepsilon} \in L^{2}\left(0, T ; \mathcal{V}^{\varepsilon}\right)$ and $p^{\varepsilon} \in L^{2}\left(0, T ; \mathcal{P}^{\varepsilon}\right)$ we shall define two bilinear forms $a^{\varepsilon}(\cdot, \cdot): L^{2}\left(0, T ; \mathcal{V}^{\varepsilon}\right) \times L^{2}\left(0, T ; \mathcal{V}^{\varepsilon}\right) \rightarrow \mathbb{R}$ and $b(\cdot, \cdot): L^{2}\left(0, T ; \mathcal{V}^{\varepsilon}\right) \times L^{2}\left(0, T ; \mathcal{P}^{\varepsilon}\right) \rightarrow \mathbb{R}:$

$$
\begin{aligned}
a^{\varepsilon}(\varphi, \psi) & =\varepsilon^{2}\left\langle 2 \eta \mathrm{S} \varphi_{1}, \mathrm{~S} \psi_{1}\right\rangle_{\Omega_{z}^{\varepsilon}, T}+\left\langle K_{a, \varepsilon}^{-1} \varphi_{2}, \psi_{2}\right\rangle_{\Omega_{a}^{\varepsilon}, T}+\left\langle K_{s p, \varepsilon}^{-1} \varphi_{3}, \psi_{3}\right\rangle_{\Omega_{a s}^{\varepsilon}, T}+\varepsilon\left\langle\kappa^{\varepsilon} \varphi_{2} \cdot \boldsymbol{n}, \psi_{2} \cdot \boldsymbol{n}\right\rangle_{\Gamma_{z s}^{\varepsilon}, T}, \\
b(\psi, q) & =\left\langle\operatorname{div} \psi_{1}, q_{1}\right\rangle_{\Omega_{z}^{\varepsilon}, T}+\left\langle\operatorname{div} \psi_{2}, q_{2}\right\rangle_{\Omega_{a}^{\varepsilon}, T}+\left\langle\operatorname{div} \psi_{3}, q_{3}\right\rangle_{\Omega_{a s}^{\varepsilon}, T},
\end{aligned}
$$

for $\varphi, \psi \in L^{2}\left(0, T ; \mathcal{V}^{\varepsilon}\right)$ and $q \in L^{2}\left(0, T ; \mathcal{P}^{\varepsilon}\right)$.

For $c_{s}^{\varepsilon}, c_{a}^{\varepsilon} \in L^{2}\left((0, T) \times \Gamma_{z s}^{\varepsilon}\right)$ we define a linear form $f^{\varepsilon}(\cdot): L^{2}\left(0, T ; \mathcal{V}^{\varepsilon}\right) \rightarrow \mathbb{R}$

$$
f^{\varepsilon}(\psi)=\varepsilon\left\langle\delta^{\varepsilon}\left(c_{s}^{\varepsilon}-c_{a}^{\varepsilon}\right), \psi_{2} \cdot \boldsymbol{n}\right\rangle_{\Gamma_{z s}^{\varepsilon}, T} \quad \text { for } \psi \in L^{2}\left(0, T ; \mathcal{V}^{\varepsilon}\right) .
$$


Theorem 4.3. For $c_{a}^{\varepsilon}, c_{s}^{\varepsilon} \in L^{2}\left((0, T) \times \Gamma_{z s}^{\varepsilon}\right)$ and $K_{a}, K_{s p}, v_{D}$ satisfying Assumption 1 and for any $\varepsilon>0$ there exists a unique solution $\left(\boldsymbol{v}^{\varepsilon}-\bar{V}_{D}^{\varepsilon}\right) \in L^{2}\left(0, T ; \mathcal{V}^{\varepsilon}\right), p^{\varepsilon} \in L^{2}\left(0, T ; \mathcal{P}^{\varepsilon}\right)$ of $(3.1)$ with transmission conditions (3.8) and boundary conditions (3.10) satisfying

$$
\begin{aligned}
& \varepsilon\left\|\nabla \boldsymbol{v}_{z}^{\varepsilon}\right\|_{L^{2}\left((0, T) \times \Omega_{z}^{\varepsilon}\right)}+\left\|\boldsymbol{v}_{s}^{\varepsilon}\right\|_{L^{2}\left((0, T) \times \Omega_{s}^{\varepsilon}\right)}+\left\|\boldsymbol{v}_{a}^{\varepsilon}\right\|_{L^{2}\left((0, T) \times \Omega_{a}^{\varepsilon}\right)}+\varepsilon^{\frac{1}{2}}\left\|\boldsymbol{v}_{a}^{\varepsilon} \cdot \boldsymbol{n}\right\|_{L^{2}\left((0, T) \times \Gamma_{z s}^{\varepsilon}\right)} \\
& +\left\|p_{s}^{\varepsilon}\right\|_{L^{2}\left((0, T) \times \Omega_{s}^{\varepsilon}\right)}+\left\|p_{a}^{\varepsilon}\right\|_{L^{2}\left((0, T) \times \Omega_{a}^{\varepsilon}\right)} \leq C \varepsilon^{\frac{1}{2}}\left(\left\|c_{a}^{\varepsilon}\right\|_{L^{2}\left((0, T) \times \Gamma_{z s}^{\varepsilon}\right)}+\left\|c_{s}^{\varepsilon}\right\|_{L^{2}\left((0, T) \times \Gamma_{z s}^{\varepsilon}\right)}\right)+C\left\|V_{D}\right\|_{H^{1}(\Omega)},
\end{aligned}
$$

and there exist extensions $P_{s}^{\varepsilon}$ of $p_{s}^{\varepsilon}$ from $\Omega_{s}^{\varepsilon}$ into $\Omega$ and $P_{a}^{\varepsilon}$ of $p_{a}^{\varepsilon}$ from $\Omega_{a}^{\varepsilon}$ into $\Omega$, satisfying $\left\|P_{s}^{\varepsilon}\right\|_{L^{2}\left(0, T ; L^{2}(\Omega) / \mathbb{R}\right)}+\left\|P_{a}^{\varepsilon}\right\|_{L^{2}\left(0, T ; L^{2}(\Omega) / \mathbb{R}\right)} \leq C \varepsilon^{\frac{1}{2}}\left(\left\|c_{a}^{\varepsilon}\right\|_{L^{2}\left((0, T) \times \Gamma_{z s}^{\varepsilon}\right)}+\left\|c_{s}^{\varepsilon}\right\|_{L^{2}\left((0, T) \times \Gamma_{z s}^{\varepsilon}\right)}\right)+C\left\|V_{D}\right\|_{H^{1}(\Omega)}$, where a universal constant $C$ is independent of $\varepsilon$ and $\Omega_{T}=\Omega \times(0, T)$.

Additionally for $c_{a, i}^{\varepsilon}, c_{s, i}^{\varepsilon} \in L^{2}\left((0, T) \times \Gamma_{z s}^{\varepsilon}\right)$, with $i=1,2$, holds

$$
\begin{aligned}
\left\|\boldsymbol{v}_{s, 1}^{\varepsilon}-\boldsymbol{v}_{s, 2}^{\varepsilon}\right\|_{L^{2}\left((0, T) \times \Omega_{s}^{\varepsilon}\right)+} & \left\|\boldsymbol{v}_{a, 1}^{\varepsilon}-\boldsymbol{v}_{a, 2}^{\varepsilon}\right\|_{L^{2}\left((0, T) \times \Omega_{a}^{\varepsilon}\right)} \\
& \leq C\left(\varepsilon^{\frac{1}{2}}\left\|c_{a, 1}^{\varepsilon}-c_{a, 2}^{\varepsilon}\right\|_{L^{2}\left((0, T) \times \Gamma_{z s}^{\varepsilon}\right)}+\varepsilon^{\frac{1}{2}}\left\|c_{s, 1}^{\varepsilon}-c_{s, 2}^{\varepsilon}\right\|_{L^{2}\left((0, T) \times \Gamma_{z s}^{\varepsilon}\right)}\right) .
\end{aligned}
$$

Proof. We can reformulate Stokes-Darcy problem (4.1) as

$$
\begin{cases}a^{\varepsilon}\left(\boldsymbol{v}^{\varepsilon}-\bar{V}_{D}^{\varepsilon}, \psi\right)+b\left(\psi, p^{\varepsilon}\right)=-a^{\varepsilon}\left(\bar{V}_{D}^{\varepsilon}, \psi\right)-f^{\varepsilon}(\psi) & \text { for } \psi \in L^{2}\left(0, T ; \mathcal{V}^{\varepsilon}\right), \\ b\left(\boldsymbol{v}^{\varepsilon}, q\right)=0 & \text { for } q \in L^{2}\left(0, T ; \mathcal{P}^{\varepsilon}\right)\end{cases}
$$

and apply the abstract theory of mixed problems, [17], to show the existence of a unique solution of (4.15). Considering $\psi \in L^{2}\left(0, T ; \mathcal{V}_{d}^{\varepsilon}\right)$, using $\psi_{1} \times \boldsymbol{n}=0$ on $(0, T) \times \Gamma_{z s}^{\varepsilon}$ and applying inequality (4.4), we obtain

$$
\begin{aligned}
a^{\varepsilon}(\psi, \psi) & \geq C\left(\varepsilon^{2}\left\|\mathrm{~S} \psi_{1}\right\|_{L^{2}\left((0, T) \times \Omega_{z}^{\varepsilon}\right)}^{2}+\left\|\psi_{2}\right\|_{L^{2}\left((0, T) \times \Omega_{a}^{\varepsilon}\right)}^{2}+\left\|\psi_{3}\right\|_{L^{2}\left((0, T) \times \Omega_{a s}^{\varepsilon}\right)}^{2}+\varepsilon\left\|\psi_{2} \cdot \boldsymbol{n}\right\|_{L^{2}\left((0, T) \times \Gamma_{z s}^{\varepsilon}\right)}^{2}\right) \\
& \geq C\|\psi\|_{L^{2}\left(0, T ; \mathcal{V}^{\varepsilon}\right)}^{2},
\end{aligned}
$$

and conclude that $a^{\varepsilon}(\cdot, \cdot)$ is $L^{2}\left(0, T ; \mathcal{V}_{d}^{\varepsilon}\right)$-elliptic.

The bilinear forms $a^{\varepsilon}(\cdot, \cdot)$ and $b(\cdot, \cdot)$ are continuous with constants independent of $\varepsilon$, i.e. for $\psi, \varphi \in$ $L^{2}\left(0, T ; \mathcal{V}^{\varepsilon}\right)$ and $q \in L^{2}\left(0, T ; \mathcal{P}^{\varepsilon}\right)$, applying Hölder's inequality, we have

$$
\begin{aligned}
\left|a^{\varepsilon}(\psi, \varphi)\right| \leq & C\left(\varepsilon^{2}\left\|\mathrm{~S} \psi_{1}\right\|_{L^{2}\left((0, T) \times \Omega_{z}^{\varepsilon}\right)}\left\|\mathrm{S} \varphi_{1}\right\|_{L^{2}\left((0, T) \times \Omega_{z}^{\varepsilon}\right)}+\left\|\psi_{2}\right\|_{L^{2}\left((0, T) \times \Omega_{a}^{\varepsilon}\right)}\left\|\varphi_{2}\right\|_{L^{2}\left((0, T) \times \Omega_{a}^{\varepsilon}\right)}\right. \\
& \left.+\left\|\psi_{3}\right\|_{L^{2}\left((0, T) \times \Omega_{a s}^{\varepsilon}\right)}\left\|\varphi_{3}\right\|_{L^{2}\left((0, T) \times \Omega_{a s}^{\varepsilon}\right)}+\varepsilon\left\|\psi_{2} \cdot \boldsymbol{n}\right\|_{L^{2}\left((0, T) \times \Gamma_{z s}^{\varepsilon}\right)}\left\|\varphi_{2} \cdot \boldsymbol{n}\right\|_{L^{2}\left((0, T) \times \Gamma_{z s}^{\varepsilon}\right)}\right) \\
\leq & C\|\psi\|_{L^{2}\left(0, T ; \mathcal{V}^{\varepsilon}\right)}\|\varphi\|_{L^{2}\left(0, T ; \mathcal{V}^{\varepsilon}\right)}
\end{aligned}
$$

and

$$
\begin{aligned}
|b(\psi, q)| \leq( & \left.\left\|\operatorname{div} \psi_{1}\right\|_{L^{2}\left((0, T) \times \Omega_{z}^{\varepsilon}\right)}+\left\|\operatorname{div} \psi_{2}\right\|_{L^{2}\left((0, T) \times \Omega_{a}^{\varepsilon}\right)}+\left\|\operatorname{div} \psi_{3}\right\|_{L^{2}\left((0, T) \times \Omega_{a s}^{\varepsilon}\right)}\right) \times \\
& \times\left(\left\|q_{1}\right\|_{L^{2}\left((0, T) \times \Omega_{z}^{\varepsilon}\right)}+\left\|q_{2}\right\|_{L^{2}\left((0, T) \times \Omega_{a}^{\varepsilon}\right)}+\left\|q_{3}\right\|_{L^{2}\left((0, T) \times \Omega_{a s}^{\varepsilon}\right)}\right) \leq\|\psi\|_{L^{2}\left(0, T ; \mathcal{V}^{\varepsilon}\right)}\|q\|_{L^{2}\left(0, T ; \mathcal{P}^{\varepsilon}\right)} .
\end{aligned}
$$

Now we shall prove that $b(\cdot, \cdot)$ satisfies the inf-sup condition. For any $q \in L^{2}\left(0, T ; \mathcal{P}^{\varepsilon}\right) \backslash\{0\}$ we shall construct $\psi \in L^{2}\left(0, T ; \mathcal{V}^{\varepsilon}\right) \backslash\{0\}$ such that

$$
b(\psi, q) \geq C\|\psi\|_{L^{2}\left(0, T ; \mathcal{V}^{\varepsilon}\right)}\|q\|_{L^{2}\left(0, T ; \mathcal{P}^{\varepsilon}\right)} .
$$

For given $q=\left(q_{1}, q_{2}, q_{3}\right) \in L^{2}\left(0, T ; \mathcal{P}^{\varepsilon}\right)$ we define $\tilde{q}_{1} \in L^{2}((0, T) \times \Omega)$ and $\tilde{q}_{2} \in L^{2}\left((0, T) \times \Omega_{s}^{\varepsilon}\right)$ as

$$
\begin{array}{ll}
\tilde{q}_{1}=q_{2} \text { in }(0, T) \times \Omega_{a}^{\varepsilon}, & \tilde{q}_{1}=q_{1}+\frac{1}{\left|\Omega_{z}^{\varepsilon}\right|}\left\langle q_{3}, 1\right\rangle_{\Omega_{a s}^{\varepsilon}} \text { in }(0, T) \times \Omega_{z}^{\varepsilon}, \\
\tilde{q}_{2}=q_{3} \text { in }(0, T) \times \Omega_{a s}^{\varepsilon}, & \tilde{q}_{2}=-\frac{1}{\left|\Omega_{z}^{\varepsilon}\right|}\left\langle q_{3}, 1\right\rangle_{\Omega_{a s}^{\varepsilon}} \quad \text { in }(0, T) \times \Omega_{z}^{\varepsilon} .
\end{array}
$$


Then, due to $\left\langle\tilde{q}_{1}, 1\right\rangle_{\Omega}=0$ and $\left\langle\tilde{q}_{2}, 1\right\rangle_{\Omega_{s}^{\varepsilon}}=0$, there exist $\tilde{\psi}_{1} \in L^{2}\left(0, T ; H_{0}^{1}(\Omega)\right)$ and $\tilde{\psi}_{2} \in L^{2}\left(0, T ; H^{1}\left(\Omega_{s}^{\varepsilon}\right)\right)$, see [17, Corollary 2.4, Lemma 2.2] or [39, Lemma 2.4], such that

$$
\begin{array}{rlrlrl}
\operatorname{div} \tilde{\psi}_{1}=\tilde{q}_{1} & \text { in } \Omega \times(0, T), & \tilde{\psi}_{1} & =0 & & \text { on } \partial \Omega \times(0, T), \\
\operatorname{div} \tilde{\psi}_{2}=\tilde{q}_{2} & \text { in } \Omega_{s}^{\varepsilon} \times(0, T), & \tilde{\psi}_{2} \cdot \boldsymbol{n} & =0 & & \text { on } \partial \Omega_{s}^{\varepsilon} \times(0, T), \\
\tilde{\psi}_{2} \times \boldsymbol{n} & =-\tilde{\psi}_{1} \times \boldsymbol{n} & & \text { on } \Gamma_{z s}^{\varepsilon} \times(0, T),
\end{array}
$$

and satisfy estimates

$$
\begin{aligned}
\left\|\tilde{\psi}_{1}\right\|_{L^{2}\left(0, T ; H^{1}(\Omega)\right)} & \leq C\left\|\tilde{q}_{1}\right\|_{L^{2}((0, T) \times \Omega)} \leq C\left(\left\|q_{1}\right\|_{L^{2}\left((0, T) \times \Omega_{z}^{\varepsilon}\right)}+\left\|q_{2}\right\|_{L^{2}\left((0, T) \times \Omega_{a}^{\varepsilon}\right)}+\left\|q_{3}\right\|_{L^{2}\left((0, T) \times \Omega_{a s}^{\varepsilon}\right)}\right), \\
\left\|\tilde{\psi}_{2}\right\|_{L^{2}\left(0, T ; H^{1}\left(\Omega_{s}^{\varepsilon}\right)\right)} & \leq C(\varepsilon)\left\|\tilde{q}_{2}\right\|_{L^{2}\left((0, T) \times \Omega_{s}^{\varepsilon}\right)} \leq C(\varepsilon)\left\|q_{3}\right\|_{L^{2}\left((0, T) \times \Omega_{a s}^{\varepsilon}\right)} .
\end{aligned}
$$

Notice that $\tilde{\psi}_{1} \in L^{2}\left(0, T \sim H_{0}^{1}(\Omega)\right)$ ensures $\tilde{\psi}_{1} \times \boldsymbol{n} \in L^{2}\left(0, T ; H^{1 / 2}\left(\Gamma_{z s}^{\varepsilon}\right)\right)$ and, therefore, also the existence of $\tilde{\psi}_{2}$ with $\tilde{\psi}_{2} \times \boldsymbol{n}=-\tilde{\psi}_{1} \times \boldsymbol{n}$ on $\Gamma_{z s}^{\varepsilon} \times(0, T)$, whereas $t \in(0, T)$ plays the role of a parameter.

We define $\psi=\left(\psi_{1}, \psi_{2}, \psi_{3}\right)$ by $\psi_{1}=\left.\left(\tilde{\psi}_{1}+\tilde{\psi}_{2}\right)\right|_{\Omega_{z}^{\varepsilon}}, \quad \psi_{2}=\left.\tilde{\psi}_{1}\right|_{\Omega_{a}^{\varepsilon}}, \quad \psi_{3}=\left.\tilde{\psi}_{2}\right|_{\Omega_{a s}^{\varepsilon}}$. The definitions of $\tilde{\psi}_{1}$ and $\tilde{\psi}_{2}$ imply that $\psi_{2} \cdot \boldsymbol{n}=0$ on $\partial \Omega \times(0, T)$ and $\psi_{3} \cdot \boldsymbol{n}=0$ on $\left(\partial \Omega_{s}^{\varepsilon} \cap \partial \Omega\right) \times(0, T)$. The properties $\tilde{\psi}_{1} \in L^{2}\left(0, T ; H^{1}(\Omega)\right)$ and $\tilde{\psi}_{2} \cdot \boldsymbol{n}=0$ on $\partial \Omega_{s}^{\varepsilon} \times(0, T)$ and $\tilde{\psi}_{2} \times \boldsymbol{n}=-\tilde{\psi}_{1} \times \boldsymbol{n}$ on $\Gamma_{z s}^{\varepsilon} \times(0, T)$ ensure $\psi_{1} \cdot \boldsymbol{n}=\psi_{2} \cdot \boldsymbol{n}$ on $\Gamma_{z}^{\varepsilon} \times(0, T)$ and $\psi_{1} \times \boldsymbol{n}=0$ on $\Gamma_{z s}^{\varepsilon} \times(0, T)$, as well as $\psi_{1} \cdot \boldsymbol{n}=\left(\psi_{2}+\psi_{3}\right) \cdot \boldsymbol{n}$ on $\Gamma_{a s}^{\varepsilon} \times(0, T)$ and $\psi_{3} \cdot \boldsymbol{n}=0$ on $\Gamma_{a w}^{\varepsilon} \times(0, T)$. The regularity of $\tilde{\psi}_{1}$ and $\tilde{\psi}_{2}$ implies also that $\psi_{1} \in L^{2}\left(0, T ; H^{1}\left(\Omega_{z}^{\varepsilon}\right)\right)$, $\psi_{2} \in L^{2}\left(0, T ; H\left(\operatorname{div}, \Omega_{a}^{\varepsilon}\right)\right)$ and $\psi_{3} \in L^{2}\left(0, T ; H\left(\operatorname{div}, \Omega_{a s}^{\varepsilon}\right)\right)$. Thus $\psi=\left(\psi_{1}, \psi_{2}, \psi_{2}\right) \in W^{\varepsilon} \cap L^{2}\left(0, T ; \mathcal{V}^{\varepsilon}\right)$ with $W^{\varepsilon}=L^{2}\left(0, T ; H^{1}\left(\Omega_{z}^{\varepsilon}\right)\right) \times L^{2}\left(0, T ; H^{1}\left(\Omega_{a}^{\varepsilon}\right)\right) \times L^{2}\left(0, T ; H^{1}\left(\Omega_{a s}^{\varepsilon}\right)\right)$ and $W^{\varepsilon} \cap L^{2}\left(0, T ; \mathcal{V}^{\varepsilon}\right)$ is continuously embedded in $L^{2}\left(0, T ; \mathcal{V}^{\varepsilon}\right)$ and for $\psi \in W^{\varepsilon} \cap L^{2}\left(0, T ; \mathcal{V}^{\varepsilon}\right)$ we have

$$
\|\psi\|_{L^{2}\left(0, T ; \mathcal{V}^{\varepsilon}\right)} \leq C\|\psi\|_{W^{\varepsilon}} \leq C(\varepsilon)\left(\left\|\tilde{q}_{1}\right\|_{L^{2}((0, T) \times \Omega)}+\left\|\tilde{q}_{2}\right\|_{L^{2}\left((0, T) \times \Omega_{s}^{\varepsilon}\right)}\right) \leq C(\varepsilon)\|q\|_{L^{2}\left(0, T ; \mathcal{P}^{\varepsilon}\right)} .
$$

Here we used that $q_{1}=\left.\left(\tilde{q}_{1}+\tilde{q}_{2}\right)\right|_{\Omega_{z}^{\varepsilon}}, q_{2}=\left.\tilde{q}_{1}\right|_{\Omega_{a}^{\varepsilon}}$ and $q_{3}=\left.\tilde{q}_{2}\right|_{\Omega_{a s}^{\varepsilon}}$. Thus, the definition of $b(\cdot, \cdot)$ yields

$$
\begin{aligned}
b(\psi, q) & =\left\langle\tilde{q}_{1}+\tilde{q}_{2}, \operatorname{div}\left(\tilde{\psi}_{1}+\tilde{\psi}_{2}\right)\right\rangle_{\Omega_{z}^{\varepsilon}, T}+\left\langle\tilde{q}_{1}, \operatorname{div} \tilde{\psi}_{1}\right\rangle_{\Omega_{a}^{\varepsilon}, T}+\left\langle\tilde{q}_{2}, \operatorname{div} \tilde{\psi}_{2}\right\rangle_{\Omega_{a s}^{\varepsilon}, T} \\
& =\left\|q_{1}\right\|_{L^{2}\left((0, T) \times \Omega_{z}^{\varepsilon}\right)}^{2}+\left\|q_{2}\right\|_{L^{2}\left((0, T) \times \Omega_{a}^{\varepsilon}\right)}^{2}+\left\|q_{3}\right\|_{L^{2}\left((0, T) \times \Omega_{a s}^{\varepsilon}\right)}^{2} \geq C_{1}(\varepsilon)\left(\left\|\tilde{\psi}_{1}\right\|_{L^{2}\left(0, T ; H^{1}(\Omega)\right)}\right. \\
& \left.+\left\|\tilde{\psi}_{2}\right\|_{L^{2}\left((0, T) \times \Omega_{s}^{\varepsilon}\right)}+\left\|\operatorname{div} \tilde{\psi}_{2}\right\|_{L^{2}\left((0, T) \times \Omega_{s}^{\varepsilon}\right)}+\varepsilon\left\|\nabla \tilde{\psi}_{2}\right\|_{L^{2}\left((0, T) \times \Omega_{s}^{\varepsilon}\right)}\right)\left(\left\|q_{1}\right\|_{L^{2}\left((0, T) \times \Omega_{z}^{\varepsilon}\right)}\right. \\
& \left.+\left\|q_{2}\right\|_{L^{2}\left((0, T) \times \Omega_{a}^{\varepsilon}\right)}+\left\|q_{3}\right\|_{L^{2}\left((0, T) \times \Omega_{a s}^{\varepsilon}\right)}\right) \geq C_{2}(\varepsilon)\|\psi\|_{L^{2}\left(0, T ; \mathcal{V}^{\varepsilon}\right)}\|q\|_{L^{2}\left(0, T ; \mathcal{P}^{\varepsilon}\right)},
\end{aligned}
$$

and $b(\cdot, \cdot)$ satisfies the inf-sup condition

$$
\inf _{q \in L^{2}\left(0, T ; \mathcal{P}^{\varepsilon}\right), q \neq 0} \sup _{\psi \in L^{2}\left(0, T ; \mathcal{V}^{\varepsilon}\right), \psi \neq 0} \frac{b(\psi, q)}{\|\psi\|_{L^{2}\left(0, T ; \mathcal{V}^{\varepsilon}\right)}\|q\|_{L^{2}\left(0, T ; \mathcal{P}^{\varepsilon}\right)}} \geq C>0 .
$$

The regularity of $c_{s}^{\varepsilon}, c_{a}^{\varepsilon}$ and Hölder's inequality imply the boundedness in $L^{2}\left(0, T ; \mathcal{V}^{\varepsilon}\right)$ of $f^{\varepsilon}(\cdot)$

$$
\begin{aligned}
\left|f^{\varepsilon}(\psi)\right| & \leq C \varepsilon\left(\left\|c_{a}^{\varepsilon}\right\|_{L^{2}\left((0, T) \times \Gamma_{z s}^{\varepsilon}\right)}+\left\|c_{s}^{\varepsilon}\right\|_{L^{2}\left((0, T) \times \Gamma_{z s}^{\varepsilon}\right)}\right)\left\|\psi_{2}\right\|_{L^{2}\left((0, T) \times \Gamma_{z s}^{\varepsilon}\right)} \\
& \leq C \varepsilon^{1 / 2}\left(\left\|c_{a}^{\varepsilon}\right\|_{L^{2}\left((0, T) \times \Gamma_{z s}^{\varepsilon}\right)}+\left\|c_{s}^{\varepsilon}\right\|_{L^{2}\left((0, T) \times \Gamma_{z s}^{\varepsilon}\right)}\right)\|\psi\|_{L^{2}\left(0, T ; \mathcal{V}^{\varepsilon}\right)} .
\end{aligned}
$$

The uniform boundedness of $V_{D}^{\varepsilon}$ in $L^{2}\left(\Omega_{a}^{\varepsilon}\right)$ and estimate (4.16) ensure the boundedness of the linear form $a^{\varepsilon}\left(\bar{V}_{D}^{\varepsilon}, \cdot\right)$. Combining all estimates and applying Corollary I.4.1 in [17] imply the existence of a unique solution $\left(u^{\varepsilon}, p^{\varepsilon}\right) \in L^{2}\left(0, T ; \mathcal{V}^{\varepsilon}\right) \times L^{2}\left(0, T ; \mathcal{P}^{\varepsilon}\right)$ of $(4.1)$ for given $c_{a}^{\varepsilon}, c_{s}^{\varepsilon} \in L^{2}\left((0, T) \times \Gamma_{z s}^{\varepsilon}\right)$.

For the proof of a priori estimates (4.12) we start with the estimates for $\boldsymbol{v}_{z}^{\varepsilon}$, $\boldsymbol{v}_{a}^{\varepsilon}$ and $\boldsymbol{v}_{s p}^{\varepsilon}$. We consider $\psi_{1}=\boldsymbol{v}_{z}^{\varepsilon}$ in $\Omega_{z}^{\varepsilon} \times(0, T)$ and $\psi_{2}=\boldsymbol{v}_{a}^{\varepsilon}-V_{D}^{\varepsilon}$ in $\Omega_{a}^{\varepsilon} \times(0, T)$, and $\psi_{3}=\boldsymbol{v}_{s p}^{\varepsilon}$ in $\Omega_{a s}^{\varepsilon} \times(0, T)$ as test functions in (4.1) and, using the divergence-free property, obtain

$$
\begin{aligned}
\varepsilon^{2}\left\|\mathrm{~S} \boldsymbol{v}_{z}^{\varepsilon}\right\|_{L^{2}\left((0, T) \times \Omega_{z}^{\varepsilon}\right)}^{2}+ & \left\|\boldsymbol{v}_{s p}^{\varepsilon}\right\|_{L^{2}\left((0, T) \times \Omega_{a s}^{\varepsilon}\right)}^{2}+\left\|\boldsymbol{v}_{a}^{\varepsilon}\right\|_{L^{2}\left((0, T) \times \Omega_{a}^{\varepsilon}\right)}^{2}+\varepsilon\left\|\boldsymbol{v}_{a}^{\varepsilon} \cdot \boldsymbol{n}\right\|_{L^{2}\left((0, T) \times \Gamma_{z s}^{\varepsilon}\right)}^{2} \\
& \leq C \varepsilon\left(\left\|c_{s}^{\varepsilon}\right\|_{L^{2}\left((0, T) \times \Gamma_{z s}^{\varepsilon}\right)}^{2}+\left\|c_{a}^{\varepsilon}\right\|_{L^{2}\left((0, T) \times \Gamma_{z s}^{\varepsilon}\right)}^{2}\right)+C\left\|V_{D}^{\varepsilon}\right\|_{L^{2}\left(\Omega_{a}^{\varepsilon}\right)}^{2} .
\end{aligned}
$$


This together with $\left\|V_{D}^{\varepsilon}\right\|_{L^{2}\left(\Omega_{a}^{\varepsilon}\right)} \leq C\left\|V_{D}\right\|_{H^{1}(\Omega)}$, inequality (4.4) and $\boldsymbol{v}_{z}^{\varepsilon} \times \boldsymbol{n}=0$ on $\Gamma_{z s}^{\varepsilon} \times(0, T)$ imply the estimates for $\boldsymbol{v}_{i}^{\varepsilon}$, with $i=z, a$, or $s p$, stated in (4.12).

For $c_{a, i}^{\varepsilon}$ and $c_{s, i}^{\varepsilon}$ and corresponding $\boldsymbol{v}_{i}^{\varepsilon}$ with $i=1,2$, the linearity of the problem, the coercivity of $a^{\varepsilon}(\cdot, \cdot)$ and the boundedness of $f^{\varepsilon}(\cdot)$ give

$$
\left\|\boldsymbol{v}_{1}^{\varepsilon}-\boldsymbol{v}_{2}^{\varepsilon}\right\|_{L^{2}\left(0, T ; \mathcal{V}_{d}^{\varepsilon}\right)}^{2} \leq C \varepsilon\left(\left\|c_{a, 1}^{\varepsilon}-c_{a, 2}^{\varepsilon}\right\|_{L^{2}\left((0, T) \times \Gamma_{z s}^{\varepsilon}\right)}^{2}+\left\|c_{s, 1}^{\varepsilon}-c_{s, 2}^{\varepsilon}\right\|_{L^{2}\left((0, T) \times \Gamma_{z s}^{\varepsilon}\right)}^{2}\right) .
$$

This will ensure the uniqueness of a weak solution of the coupled problem (3.1)-(3.10).

By Lemma 4 in [38] there exists a restriction operator $R_{\Omega_{a}^{\varepsilon}}^{\varepsilon}: H_{0}^{1}(\Omega) \rightarrow H_{0}^{1}\left(\Omega_{a}^{\varepsilon}\right)$

$$
\begin{cases}\psi \in H_{0}^{1}\left(\Omega_{a}^{\varepsilon}\right) & \Longrightarrow R_{\Omega_{a}^{\varepsilon}}^{\varepsilon} \psi=\psi \\ \operatorname{div} \psi=0 & \Longrightarrow \operatorname{div} R_{\Omega_{a}^{\varepsilon}}^{\varepsilon} \psi=0 \\ \left\|R_{\Omega_{a}^{\varepsilon}}^{\varepsilon} \psi\right\|_{L^{2}\left(\Omega_{a}^{\varepsilon}\right)} & \leq C\left(\|\psi\|_{L^{2}(\Omega)}+\varepsilon\|\nabla \psi\|_{L^{2}(\Omega)}\right) \\ \varepsilon\left\|\nabla R_{\Omega_{a}^{\varepsilon}}^{\varepsilon} \psi\right\|_{L^{2}\left(\Omega_{a}^{\varepsilon}\right)} \leq C\left(\|\psi\|_{L^{2}(\Omega)}+\varepsilon\|\nabla \psi\|_{L^{2}(\Omega)}\right)\end{cases}
$$

As in [3, Theorem 2.3], to define $R_{\Omega_{s}^{\varepsilon}}^{\varepsilon}: H_{0}^{1}(\Omega) \rightarrow V\left(\Omega_{s}^{\varepsilon}\right)$, with $V\left(\Omega_{s}^{\varepsilon}\right)=\left\{v \in H_{0}^{1}\left(\Omega_{s}^{\varepsilon}\right): v \times \boldsymbol{n}=0\right.$ on $\left.\Gamma_{a s}^{\varepsilon}\right\}$, such that

$$
\begin{cases}\psi \in V\left(\Omega_{s}^{\varepsilon}\right) & \Longrightarrow R_{\Omega_{s}^{\varepsilon}}^{\varepsilon} \psi=\psi, \\ \operatorname{div} \psi=0 & \Longrightarrow \operatorname{div} R_{\Omega_{s}^{\varepsilon}}^{\varepsilon} \psi=0, \\ \left\|R_{\Omega_{s}^{\varepsilon}}^{\varepsilon} \psi\right\|_{L^{2}\left(\Omega_{s}^{\varepsilon}\right)} & \leq C\left(\|\psi\|_{L^{2}(\Omega)}+\varepsilon\|\nabla \psi\|_{L^{2}(\Omega)}\right) \\ \varepsilon\left\|\nabla R_{\Omega_{s}^{\varepsilon}}^{\varepsilon} \psi\right\|_{L^{2}\left(\Omega_{s}^{\varepsilon}\right)} & \leq C\left(\|\psi\|_{L^{2}(\Omega)}+\varepsilon\|\nabla \psi\|_{L^{2}(\Omega)}\right)\end{cases}
$$

we consider for $u \in H^{1}(Y)$, as in [3, Lemma 3.4], a linear operator $Q: H^{1}(Y) \rightarrow H^{1}(Y)$, such that $Q u=0$ in $Y_{a}$, and a modified problem

$$
\begin{cases}(w, q) \in H^{1}\left(Y_{s}\right) \times L^{2}\left(Y_{s}\right) / \mathbb{R}, & \text { in } Y_{z} \cup Y_{a s}, \\ -\eta \Delta w+\nabla q=\Delta u & \text { in } Y_{s}, \\ \operatorname{div} w=\operatorname{div} u+\frac{1}{\left|Y_{s}\right|} \int_{Y_{a w}} \operatorname{div} u d y & \text { on } \Gamma_{a s}, \\ w \times \boldsymbol{n}=0, \quad[w \cdot \boldsymbol{n}]=0, \quad[-2 \eta(\mathrm{S} w \boldsymbol{n}) \cdot \boldsymbol{n}+q]=0 & \text { on } \partial Y_{s, i} \cap \partial Y, \\ w=Q u+\left[\frac{\zeta_{i}}{\left\langle\zeta_{i}, 1\right\rangle_{\partial Y_{i}}} \int_{\partial Y_{i}}(u-Q u) \cdot e_{i} d y\right] e_{i} & \text { on } \partial Y_{s} \backslash \partial Y, \\ w=0 & \end{cases}
$$

where $\partial Y_{i}$, with $i=-3,-2,-1,1,2,3$, are the six faces of the cube $Y$, such that $\partial Y_{k}, \partial Y_{-k}$ are orthogonal to the unit vector $e_{k}$, with $k=1,2,3$. Here $\zeta_{i} \in C^{\infty}(\bar{Y})$, with $i=-3,-2,-1,1,2,3$, satisfy $\zeta_{i} \geq 0, \zeta_{i} \neq 0$ on $\partial Y_{i}, \zeta_{i} \equiv 0$ in $\bar{Y}_{a}$ and on $\partial Y_{j}$ for $j \neq i$, and $\left.\left.\zeta_{k}\right|_{\partial Y_{k}} \equiv \zeta_{-k}\right|_{\partial Y_{-k}}$ for $k=1,2,3$. The existence of such $\zeta_{i}$ is ensured by the geometrical structure of $Y_{s}$. Notice that the construction of the boundary conditions on $\partial Y_{s}$ ensures that $\int_{Y_{s}} \operatorname{div} w d y=\int_{\partial Y_{s}} w \cdot \boldsymbol{n} d y$. The existence of a unique solution of (4.22) can be shown by applying the abstract theory of mixed problems, similar as for (4.15). Then $R_{Y_{s}}$, given by $R_{Y_{s}} u=w$, belongs to $\mathcal{L}\left(H^{1}(Y) ; H^{1}\left(Y_{s}\right)\right)$ and in each cell $\varepsilon Y^{k} \subset \Omega$, with $k \in \mathbb{Z}^{3}$, we define $R_{\Omega_{s}^{\varepsilon}}^{\varepsilon} u=\left(R_{Y_{s}} u(\varepsilon y)\right)(x / \varepsilon)$ for $y \in Y^{k}$ and $x \in \varepsilon Y_{s}^{k}$, respectively.

Equations (3.1) imply that $\nabla p_{s} \in L^{2}\left(0, T ; H^{-1}\left(\Omega_{s}^{\varepsilon}\right)\right)$ and $\nabla p_{a} \in L^{2}\left(0, T ; H^{-1}\left(\Omega_{a}^{\varepsilon}\right)\right)$. We define the extensions of $\nabla p_{s}^{\varepsilon}$ and $\nabla p_{a}^{\varepsilon}$ into $\Omega$ using the duality argument and consider $\mathcal{F}_{s}^{\varepsilon} \in L^{2}\left(0, T ; H^{-1}(\Omega)\right)$ and $\mathcal{F}_{a}^{\varepsilon} \in L^{2}\left(0, T ; H^{-1}(\Omega)\right)$ given by

$$
\begin{array}{ll}
\left\langle\mathcal{F}_{s}^{\varepsilon}, \psi\right\rangle_{H^{-1}, H^{1}}=\left\langle\nabla p_{s}^{\varepsilon}, R_{\Omega_{s}^{\varepsilon}}^{\varepsilon} \psi\right\rangle_{L^{2}\left(0, T ; H^{-1}\left(\Omega_{s}^{\varepsilon}\right)\right), L^{2}\left(0, T ; H_{0}^{1}\left(\Omega_{s}^{\varepsilon}\right)\right)} & \text { for } \psi \in L^{2}\left(0, T ; H_{0}^{1}(\Omega)\right), \\
\left\langle\mathcal{F}_{a}^{\varepsilon}, \psi\right\rangle_{H^{-1}, H^{1}}=\left\langle\nabla p_{a}^{\varepsilon}, R_{\Omega_{a}^{\varepsilon}}^{\varepsilon} \psi\right\rangle_{L^{2}\left(0, T ; H^{-1}\left(\Omega_{a}^{\varepsilon}\right)\right), L^{2}\left(0, T ; H_{0}^{1}\left(\Omega_{a}^{\varepsilon}\right)\right)} & \text { for } \psi \in L^{2}\left(0, T ; H_{0}^{1}(\Omega)\right),
\end{array}
$$


where $\langle\cdot, \cdot\rangle_{H^{-1}, H^{1}}:=\langle\cdot, \cdot\rangle_{L^{2}\left(0, T ; H^{-1}(\Omega)\right), L^{2}\left(0, T ; H_{0}^{1}(\Omega)\right)}$. Applying the estimates for $R_{\Omega_{s}^{\varepsilon}}^{\varepsilon}(\psi)$ and $R_{\Omega_{a}^{\varepsilon}}^{\varepsilon}(\psi)$ in (4.20) and (4.21), we obtain from (3.1) for $\psi \in L^{2}\left(0, T ; H_{0}^{1}(\Omega)\right)$ that

$$
\begin{aligned}
\left\langle\mathcal{F}_{s}^{\varepsilon}, \psi\right\rangle_{H^{-1}, H^{1}} & =\left\langle\varepsilon^{2} \eta \nabla \boldsymbol{v}_{z}^{\varepsilon}, \nabla\left(R_{\Omega_{s}^{\varepsilon}}^{\varepsilon} \psi\right)\right\rangle_{\Omega_{z}^{\varepsilon}, T}+\left\langle K_{s p}^{-1} \boldsymbol{v}_{s p}^{\varepsilon}, R_{\Omega_{s}^{\varepsilon}}^{\varepsilon} \psi\right\rangle_{\Omega_{a s}^{\varepsilon}, T} \\
& \leq C\left(\varepsilon\left\|\nabla \boldsymbol{v}_{z}^{\varepsilon}\right\|_{L^{2}\left((0, T) \times \Omega_{z}^{\varepsilon}\right)}+\left\|\boldsymbol{v}_{s p}^{\varepsilon}\right\|_{L^{2}\left((0, T) \times \Omega_{a s}^{\varepsilon}\right)}\right)\left(\|\psi\|_{L^{2}\left(\Omega_{T}\right)}+\varepsilon\|\nabla \psi\|_{L^{2}\left(\Omega_{T}\right)}\right),
\end{aligned}
$$

where $\Omega_{T}=(0, T) \times \Omega$, and

$$
\left\langle\mathcal{F}_{a}^{\varepsilon}, \psi\right\rangle_{H^{-1}, H^{1}}=\left\langle K_{a}^{-1} \boldsymbol{v}_{a}^{\varepsilon}, R_{\Omega_{a}^{\varepsilon}}^{\varepsilon} \psi\right\rangle_{\Omega_{a}^{\varepsilon}, T} \leq C\left\|\boldsymbol{v}_{a}^{\varepsilon}\right\|_{L^{2}\left((0, T) \times \Omega_{a}^{\varepsilon}\right)}\left(\|\psi\|_{L^{2}\left(\left(\Omega_{T}\right)\right.}+\varepsilon\|\nabla \psi\|_{L^{2}\left(\Omega_{T}\right)}\right) .
$$

Thus for each $\varepsilon>0$ we have that $\mathcal{F}_{a}^{\varepsilon}$ and $\mathcal{F}_{s}^{\varepsilon}$ are bounded functionals on $L^{2}\left(0, T ; H_{0}^{1}(\Omega)\right)$.

For $\psi \in L^{2}\left(0, T ; H_{0}^{1}\left(\Omega_{l}^{\varepsilon}\right)\right)$ with $\psi \times \boldsymbol{n}=0$ on $\Gamma_{a s}^{\varepsilon} \times(0, T)$, where $l=a, s$, the properties of $R_{\Omega_{l}^{\varepsilon}}^{\varepsilon}$ imply $R_{\Omega_{l}^{\varepsilon}}^{\varepsilon}(\psi)=\psi$ and $\left.\mathcal{F}_{l}^{\varepsilon}\right|_{\Omega_{l}^{\varepsilon}}=\nabla p_{l}^{\varepsilon}$. We have also $\operatorname{div} R_{\Omega_{l}^{\varepsilon}}^{\varepsilon}(\psi)=0$, for $l=a, s$, provided $\operatorname{div} \psi=0$, and the orthogonality property ensures that $\mathcal{F}_{s}^{\varepsilon}$ and $\mathcal{F}_{a}^{\varepsilon}$ are the gradients with respect to $x$ of functions in $L^{2}((0, T) \times \Omega)$. It means that $\mathcal{F}_{s}^{\varepsilon}$ and $\mathcal{F}_{a}^{\varepsilon}$ are continuations of $\nabla p_{a}^{\varepsilon}$ and $\nabla p_{s}^{\varepsilon}$ to $\Omega$, respectively, and

$$
\mathcal{F}_{s}^{\varepsilon}=\nabla P_{s}^{\varepsilon}, \quad \mathcal{F}_{a}^{\varepsilon}=\nabla P_{a}^{\varepsilon} \quad \text { with } \quad P_{s}^{\varepsilon}, P_{a}^{\varepsilon} \in L^{2}((0, T) \times \Omega) / \mathbb{R} .
$$

We have also an explicit formula for the extension $P_{l}^{\varepsilon}$, with $l=s$ or $a$, see [3,19], for $t \in(0, T)$

$$
P_{l}^{\varepsilon}(t, x)= \begin{cases}p_{l}^{\varepsilon}(t, x) & \text { in } \Omega_{l}^{\varepsilon}, \\ \frac{1}{\left|\varepsilon Y_{l}^{k}\right|} \int_{\varepsilon Y_{l}^{k}} p_{l}^{\varepsilon}(t, x) d x & \text { in } \varepsilon Y^{k} \backslash \varepsilon \overline{Y_{l}^{k}} \text { for } k \in \mathbb{Z}^{3} \text { such that } \varepsilon Y^{k} \cap \Omega \neq \emptyset .\end{cases}
$$

Applying now estimates (4.23), (4.24) together with (4.18) and (4.4), and using the estimate of $L^{2}$-norm by $H^{-1}$-norm, see $[17,39]$, give

$$
\begin{aligned}
\left\|P_{s}^{\varepsilon}\right\|_{L^{2}\left(0, T ; L^{2}(\Omega) / \mathbb{R}\right)} & \leq C_{1}\left\|P_{s}^{\varepsilon}\right\|_{L^{2}\left(0, T ; H^{-1}(\Omega)\right)} \leq C_{2}\left(\varepsilon\left\|\nabla \boldsymbol{v}_{z}^{\varepsilon}\right\|_{L^{2}\left((0, T) \times \Omega_{z}^{\varepsilon}\right)}+\left\|\boldsymbol{v}_{s p}^{\varepsilon}\right\|_{L^{2}\left((0, T) \times \Omega_{a s}^{\varepsilon}\right)}\right) \\
& \leq C_{3} \varepsilon^{1 / 2}\left(\left\|c_{s}^{\varepsilon}\right\|_{L^{2}\left((0, T) \times \Gamma_{z s}^{\varepsilon}\right)}+\left\|c_{a}^{\varepsilon}\right\|_{L^{2}\left((0, T) \times \Gamma_{z s}^{\varepsilon}\right)}\right)+C_{4}\left\|V_{D}^{\varepsilon}\right\|_{L^{2}\left(\Omega_{a}^{\varepsilon}\right)}, \\
\left\|P_{a}^{\varepsilon}\right\|_{L^{2}\left(0, T ; L^{2}(\Omega) / \mathbb{R}\right)} & \leq C_{1}\left\|P_{a}^{\varepsilon}\right\|_{L^{2}\left(0, T ; H^{-1}(\Omega)\right)} \leq C_{2}\left\|\boldsymbol{v}_{a}^{\varepsilon}\right\|_{L^{2}\left((0, T) \times \Omega_{a}^{\varepsilon}\right)} \\
& \leq C_{3} \varepsilon^{1 / 2}\left(\left\|c_{s}^{\varepsilon}\right\|_{L^{2}\left((0, T) \times \Gamma_{z s}^{\varepsilon}\right)}+\left\|c_{a}^{\varepsilon}\right\|_{L^{2}\left((0, T) \times \Gamma_{z s}^{\varepsilon}\right)}\right)+C_{4}\left\|V_{D}^{\varepsilon}\right\|_{L^{2}\left(\Omega_{a}^{\varepsilon}\right)} .
\end{aligned}
$$

The last estimates together with $\left\|V_{D}^{\varepsilon}\right\|_{L^{2}\left(\Omega_{a}^{\varepsilon}\right)} \leq C\left\|V_{D}\right\|_{H^{1}(\Omega)}$ and the definition of $P_{s}^{\varepsilon}$ and $P_{a}^{\varepsilon}$ ensure (4.13) and $L^{2}$-estimates for $p_{s}^{\varepsilon}$ and $p_{a}^{\varepsilon}$ in (4.12).

\subsection{Existence and estimates for $c_{s}^{\varepsilon}, c_{a}^{\varepsilon}, \vartheta_{f, a}^{\varepsilon}, \vartheta_{b, a}^{\varepsilon}, \vartheta_{f, s}^{\varepsilon}$ and $\vartheta_{b, s}^{\varepsilon}$}

Using classical results $[1,8]$ we can extend the domain of definition of solute concentrations $c_{l}^{\varepsilon}$ from a connected domain $\Omega_{l}^{\varepsilon}$ to $\Omega$, where $l=a, s$.

Lemma 4.4. 1. For $c_{l} \in W^{1, p}\left(Y_{l}\right)$, with $1<p<\infty$, there exists an extension $\tilde{c}_{l}$ to $Y$ such that

$$
\left\|\tilde{c}_{l}\right\|_{L^{p}(Y)} \leq \Xi\left\|c_{l}\right\|_{L^{p}\left(Y_{l}\right)} \quad \text { and } \quad\left\|\nabla \tilde{c}_{l}\right\|_{L^{p}(Y)} \leq \Xi\left\|\nabla c_{l}\right\|_{L^{p}\left(Y_{l}\right)} .
$$

2. There exists an extension $\tilde{c}_{l}^{\varepsilon}$ of $c_{l}^{\varepsilon}$ from $W^{1, p}\left(\Omega_{l}^{\varepsilon}\right)$ into $W^{1, p}(\Omega)$ such that

$$
\left\|\tilde{c}_{l}^{\varepsilon}\right\|_{L^{p}(\Omega)} \leq \Xi\left\|c_{l}^{\varepsilon}\right\|_{L^{p}\left(\Omega_{l}^{\varepsilon}\right)} \quad \text { and } \quad\left\|\nabla \tilde{c}_{l}^{\varepsilon}\right\|_{L^{p}(\Omega)} \leq \Xi\left\|\nabla c_{l}^{\varepsilon}\right\|_{L^{p}\left(\Omega_{l}^{\varepsilon}\right)},
$$

where the constant $\Xi$ depends on $Y$ and $Y_{l}$ only.

Due to the geometric assumptions on $\Omega_{a}^{\varepsilon}$, holes in the domain do not touch each other, have smooth boundary and do not touch the boundary $\partial \Omega$, i.e. $\Gamma_{z s}^{\varepsilon} \cap \partial \Omega=\emptyset$. Therefore, classical extension results $[1,8]$ apply to $c_{a}^{\varepsilon}$. Due to the structural assumptions, $\Omega_{s}^{\varepsilon}$ is a connected domain in $\mathbb{R}^{3}$ with Lipschitzcontinous boundary $\partial \Omega_{s}^{\varepsilon}$. The geometrical assumptions on $\Omega_{s}^{\varepsilon}$ ensure also that it is sufficient to extend 
$c_{s}^{\varepsilon}$ by reflection in tangential directions near the boundary $\partial \Omega_{s}^{\varepsilon} \cap \partial \Omega$. Therefore the extension results apply also for $c_{s}^{\varepsilon}$, see $[1,33]$, and the extension operator is defined globally in $\Omega$.

For $c_{l}^{\varepsilon} \in H^{1}\left(0, T ; H^{1}\left(\Omega_{l}^{\varepsilon}\right)\right)$ we define $\hat{c}_{l}^{\varepsilon}(\cdot, t):=\tilde{c}_{l}^{\varepsilon}(\cdot, t)$ a.e. in time. Since the extension operator is linear and bounded and $\Omega_{l}^{\varepsilon}$ does not depend on $t$, we obtain $\hat{c}_{l}^{\varepsilon} \in H^{1}\left(0, T ; H^{1}(\Omega)\right)$ and

$$
\left\|\partial_{t} \hat{c}_{l}^{\varepsilon}\right\|_{L^{2}\left(\Omega_{T}\right)} \leq \Xi\left\|\partial_{t} c_{l}^{\varepsilon}\right\|_{L^{2}\left((0, T) \times \Omega_{l}^{\varepsilon}\right)}, \quad\left\|\partial_{t} \nabla \hat{c}_{l}^{\varepsilon}\right\|_{L^{2}\left(\Omega_{T}\right)} \leq \Xi\left\|\partial_{t} \nabla c_{l}^{\varepsilon}\right\|_{L^{2}\left((0, T) \times \Omega_{l}^{\varepsilon}\right)},
$$

where $l=a, s$. In the sequel, we shall identify $c_{l}^{\varepsilon}$ with its extension $\hat{c}_{l}^{\varepsilon}$.

Theorem 4.5. Under Assumption 1 there exists a nonnegative unique weak solution $\left(c_{a}^{\varepsilon}, c_{s}^{\varepsilon}, \vartheta_{f, a}^{\varepsilon}, \vartheta_{b, a}^{\varepsilon}, \vartheta_{f, s}^{\varepsilon}, \vartheta_{b, s}^{\varepsilon}\right)$ of (3.2) and (3.5) with boundary conditions (3.3), (3.6), and (3.9) and initial conditions $(3.7)$ such that $c_{l}^{\varepsilon} \in H^{1}\left(0, T ; H^{1}\left(\Omega_{l}^{\varepsilon}\right)\right) \cap L^{\infty}\left((0, T) \times \Omega_{l}^{\varepsilon}\right), \vartheta_{j, l}^{\varepsilon} \in W^{1, \infty}\left(0, T ; L^{\infty}\left(\Gamma_{z s}^{\varepsilon}\right)\right)$, with $l=a, s, j=f, b$, and satisfies the estimates

$$
\begin{aligned}
& \left\|c_{l}^{\varepsilon}\right\|_{L^{\infty}\left(0, T ; L^{2}\left(\Omega_{l}^{\varepsilon}\right)\right)}+\left\|\nabla c_{l}^{\varepsilon}\right\|_{L^{2}\left((0, T) \times \Omega_{l}^{\varepsilon}\right)}+\varepsilon^{\frac{1}{2}}\left\|c_{l}^{\varepsilon}\right\|_{L^{2}\left((0, T) \times \Gamma_{z s}^{\varepsilon}\right)} \leq C, \\
& \left\|c_{l}^{\varepsilon}\right\|_{L^{\infty}((0, T) \times \Omega)} \leq C, \quad\left\|\vartheta_{j, l}^{\varepsilon}\right\|_{L^{\infty}\left((0, T) \times \Gamma_{z s}^{\varepsilon}\right)} \leq C,
\end{aligned}
$$

and

$$
\begin{aligned}
& \left\|\partial_{t} c_{l}^{\varepsilon}\right\|_{L^{\infty}\left(0, T ; L^{2}\left(\Omega_{l}^{\varepsilon}\right)\right)}+\left\|\partial_{t} \nabla c_{l}^{\varepsilon}\right\|_{L^{2}\left((0, T) \times \Omega_{l}^{\varepsilon}\right)}+\varepsilon^{\frac{1}{2}}\left\|\partial_{t} c_{l}^{\varepsilon}\right\|_{L^{2}\left((0, T) \times \Gamma_{z s}^{\varepsilon}\right)}+\varepsilon^{\frac{1}{2}}\left\|\partial_{t} \vartheta_{j, l}^{\varepsilon}\right\|_{L^{2}\left((0, T) \times \Gamma_{z s}^{\varepsilon}\right)} \leq C, \\
& \left\|\partial_{t} \boldsymbol{v}_{a}^{\varepsilon}\right\|_{L^{2}\left((0, T) \times \Omega_{a}^{\varepsilon}\right)}+\left\|\partial_{t} \boldsymbol{v}_{s}^{\varepsilon}\right\|_{L^{2}\left((0, T) \times \Omega_{s}^{\varepsilon}\right)}+\varepsilon\left\|\partial_{t} \nabla \boldsymbol{v}_{z}^{\varepsilon}\right\|_{L^{2}\left((0, T) \times \Omega_{z}^{\varepsilon}\right)}+\varepsilon^{\frac{1}{2}}\left\|\partial_{t} \boldsymbol{v}_{a}^{\varepsilon} \cdot \boldsymbol{n}\right\|_{L^{2}\left((0, T) \times \Gamma_{z s}^{\varepsilon}\right)} \leq C
\end{aligned}
$$

with $j=f, b$ and $l=a, s$, and the constant $C$ depends on $M$ and is independent of $\varepsilon$.

Proof. The existence of a solution will be proven by showing the existence of a fix point of the operator $\mathcal{B}$ defined on $\left(C\left([0, T] ; H^{\varsigma}\left(\Omega_{a}^{\varepsilon}\right)\right) \cap L^{\infty}\left((0, T) \times \Omega_{a}^{\varepsilon}\right)\right) \times\left(C\left([0, T] ; H^{\varsigma}\left(\Omega_{s}^{\varepsilon}\right)\right) \cap L^{\infty}\left((0, T) \times \Omega_{s}^{\varepsilon}\right)\right)$, with $1 / 2<\varsigma<1$, by $\left(c_{a}^{\varepsilon, n}, c_{s}^{\varepsilon, n}\right)=\mathcal{B}\left(c_{a}^{\varepsilon, n-1}, c_{s}^{\varepsilon, n-1}\right)$ given as solutions of $(4.2)$ and (4.3) with $\left(\vartheta_{f, l}^{\varepsilon, n}, \vartheta_{b, l}^{\varepsilon, n}\right)$ solving

$$
\left\{\begin{aligned}
\partial_{t} \vartheta_{f, l}^{\varepsilon, n} & =R_{l}^{\varepsilon}\left(t, x, \vartheta_{f, l}^{\varepsilon, n}\right)-\alpha_{l}^{\varepsilon}(t, x) c_{l}^{\varepsilon, n-1} \vartheta_{f, l}^{\varepsilon, n}+\beta_{l}^{\varepsilon}(t, x) \vartheta_{b, l}^{\varepsilon, n}-\gamma_{f, l}^{\varepsilon}(t, x) \vartheta_{f, l}^{\varepsilon, n} & \text { on } \Gamma_{z s}^{\varepsilon} \times(0, T), \\
\partial_{t} \vartheta_{b, l}^{\varepsilon, n}= & \alpha_{l}^{\varepsilon}(t, x) c_{l}^{\varepsilon, n-1} \vartheta_{f, l}^{\varepsilon, n}-\beta_{l}^{\varepsilon}(t, x) \vartheta_{b, l}^{\varepsilon, n}-\gamma_{b, l}^{\varepsilon}(t, x) \vartheta_{b, l}^{\varepsilon, n} & \text { on } \Gamma_{z s}^{\varepsilon} \times(0, T)
\end{aligned}\right.
$$

where $l=a, s$. For a given nonnegative $c_{l}^{n-1, \varepsilon} \in C\left([0, T] ; H^{\varsigma}\left(\Omega_{l}^{\varepsilon}\right)\right) \cap L^{\infty}\left((0, T) \times \Omega_{l}^{\varepsilon}\right)$, with $l=a, s$, due to the Lipschitz-continuity of the right-hand side of system (4.27), there exists a unique solution $\vartheta_{j, l}^{\varepsilon, n} \in C^{1}\left([0, T] ; L^{2}\left(\Gamma_{z s}^{\varepsilon}\right)\right)$ of $(4.27)$, with $j=f, b$ and $l=a, s$. Using that initial values are nonnegative, function $R_{l}^{\varepsilon}(t, x, \xi)$ is nonnegative for nonnegative $\xi$, and $c_{l}^{\varepsilon, n-1}(t, x) \geq 0$ a.e. on $[0, T] \times \Gamma_{z s}^{\varepsilon}$, we obtain $\vartheta_{j, l}^{n, \varepsilon}(t, x) \geq 0$ a.e on $[0, T] \times \Gamma_{z s}^{\varepsilon}$ for $j=f, b$ and $l=a, s$. Adding equations in (4.27) yields

$$
\partial_{t}\left(\vartheta_{f, l}^{\varepsilon, n}+\vartheta_{b, l}^{\varepsilon, n}\right)=R_{l}^{\varepsilon}\left(t, x, \vartheta_{f, l}^{\varepsilon, n}\right)-\gamma_{f, l}^{\varepsilon}(t, x) \vartheta_{f, l}^{\varepsilon, n}-\gamma_{b, l}^{\varepsilon}(t, x) \vartheta_{b, l}^{\varepsilon, n}, \quad \text { for } t \in[0, T] \text { and a.a } x \in \Gamma_{z s}^{\varepsilon},
$$

where $l=a, s$. Considering the Lipschitz continuity of $R_{l}^{\varepsilon}$, the nonnegativity of $\vartheta_{j, l}^{\varepsilon, n}$, the boundedness of initial conditions and applying Gronwall's inequality, we obtain the boundedness of $\vartheta_{j, l}^{\varepsilon, n}$ a.e. on $[0, T] \times \Gamma_{z s}^{\varepsilon}$, with $j=f, b$ and $l=a, s$. The boundedness of $c_{l}^{\varepsilon, n-1}$ and $\vartheta_{j, l}^{\varepsilon, n}$ implies also the boundedness of $\partial_{t} \vartheta_{j, l}^{\varepsilon, n}$ a.e. on $[0, T] \times \Gamma_{z s}^{\varepsilon}$, whereas $j=f, b$ and $l=a, s$.

Using Galerkin's method and a priori estimates similar to those shown below, we obtain the existence of a weak nonnegative solution $c_{l}^{\varepsilon, n} \in H^{1}\left(0, T ; H^{1}\left(\Omega_{l}^{\varepsilon}\right)\right) \cap L^{\infty}\left((0, T) \times \Omega_{l}^{\varepsilon}\right)$, for $l=s, a$, see [22]. The embedding $H^{1}\left(0, T ; H^{1}\left(\Omega_{l}^{\varepsilon}\right)\right) \subset C\left([0, T] ; H^{\varsigma}\left(\Omega_{l}^{\varepsilon}\right)\right)$, for $1 / 2<\varsigma<1$, is compact and, by virtue of the Schauder theorem, there exists a fix point of $\mathcal{B}$, a solution $\left(c_{l}^{\varepsilon}, \vartheta_{j, l}^{\varepsilon}\right)$ of the microscopic problem, where $j=f, b$ and $l=a, s$. In addition, we obtain boundedness of $c_{l}^{\varepsilon}(t, x)$ a.e. in $[0, T] \times \Omega_{l}^{\varepsilon}$ and on $[0, T] \times \Gamma_{z s}^{\varepsilon}$, and $c_{l}^{\varepsilon}(t, x) \geq 0$ a.e. in $[0, T] \times \Omega_{l}^{\varepsilon}$ and on $[0, T] \times \Gamma_{z s}^{\varepsilon}$, together with $\vartheta_{j, l}^{\varepsilon}(t, x) \geq 0$ a.e. on $[0, T] \times \Gamma_{z s}^{\varepsilon}$, where $j=f, b$ and $l=a, s$. This ensures also the boundedness of $\partial_{t} \vartheta_{j, l}^{\varepsilon}$ a.e. on $[0, T] \times \Gamma_{z s}^{\varepsilon}$, as well as by applying (4.28) the uniform in $\varepsilon$ boundedness of $\vartheta_{j, l}^{\varepsilon}$ a.e. on $[0, T] \times \Gamma_{z s}^{\varepsilon}$, and thus the last estimate in (4.25). 
Now we shall prove the non-negativity of $c_{l}^{\varepsilon, n}$, and thus of $c_{l}^{\varepsilon}$, and the a priori estimates for $c_{l}^{\varepsilon, n}$, and therefore for $c_{l}^{\varepsilon}$, uniformly in $\varepsilon$, where $l=a, s$. To show the non-negativity of $c_{l}^{\varepsilon, n}$, with $l=a, s$ we take $c_{l,-}^{\varepsilon}=\min \left\{0, c_{l}^{\varepsilon, n}\right\}$ as test functions in (4.2) and (4.3), respectively. Considering the assumptions on the coefficients and functions $F_{l}^{\varepsilon}$, and the nonnegativity of $\vartheta_{j, l}^{\varepsilon, n}$, with $l=a, s$ and $j=f, b$, terms on the right-hand side in (4.2) and (4.3) can be estimated by $C_{1}\left\|c_{l,-}^{\varepsilon}\right\|_{L^{2}\left((0, \tau) \times \Omega_{l}^{\varepsilon}\right)}$ for $\tau \in(0, T]$. Using Gagliardo-Nirenberg-inequality, i.e. for $\phi \in W^{1, q}(\Omega)$

$$
\|\phi\|_{L^{p}(\Omega)} \leq C_{G}\left(\|\phi\|_{L^{r}(\Omega)}^{\lambda}\|\nabla \phi\|_{L^{q}(\Omega)}^{(1-\lambda)}+\|\phi\|_{L^{1}(\Omega)}\right)
$$

where $1 \leq r<p$ and $1 \leq q \leq p$, with $\lambda \in[0,1]$ satisfies the relation $1 / p=\lambda(1 / r-1 / n)+(1-\lambda) 1 / q$ and $p \in[q, q n /(n-q)]$ for $q<n, p \in[q, \infty)$ for $q=n, p \in[q, \infty]$ for $q>n$, and considering extension of $c_{l,-}^{\varepsilon}$, defined in Lemma 4.4 , we can estimate the advective term by

$$
\begin{aligned}
& \left|\left\langle H_{M}\left(\boldsymbol{v}_{l}^{\varepsilon}\right) c_{l,-}^{\varepsilon}, \nabla c_{l,-}^{\varepsilon}\right\rangle_{\Omega_{l}^{\varepsilon}, \tau}\right| \leq \frac{1}{d_{l}}\left\|H_{M}\left(\boldsymbol{v}_{l}^{\varepsilon}\right)\right\|_{L^{\infty}\left(0, \tau ; L^{4}\left(\Omega_{l}^{\varepsilon}\right)\right)}^{2}\left\|c_{l,-}^{\varepsilon}\right\|_{L^{2}\left(0, \tau ; L^{4}\left(\Omega_{l}^{\varepsilon}\right)\right)}^{2}+\frac{d_{l}}{4}\left\|\nabla c_{l,-}^{\varepsilon}\right\|_{L^{2}\left((0, \tau) \times \Omega_{l}^{\varepsilon}\right)}^{2} \\
& \leq C\left(\left\|H_{M}\left(\boldsymbol{v}_{l}^{\varepsilon}\right)\right\|_{L^{\infty}\left(0, \tau ; L^{4}\left(\Omega_{l}^{\varepsilon}\right)\right)}^{8}+\left\|H_{M}\left(\boldsymbol{v}_{l}^{\varepsilon}\right)\right\|_{\left.L^{\infty}\left(0, \tau ; L^{4}\left(\Omega_{l}^{\varepsilon}\right)\right)\right)}^{2}\right)\left\|c_{l,-}^{\varepsilon}\right\|_{L^{2}\left((0, \tau) \times \Omega_{l}^{\varepsilon}\right)}^{2}+\frac{d_{l}}{2}\left\|\nabla c_{l,-}^{\varepsilon}\right\|_{L^{2}\left((0, \tau) \times \Omega_{l}^{\varepsilon}\right)}^{2}
\end{aligned}
$$

for $\tau \in(0, T]$. Considering uniform boundedness of $\left\|H_{M}\left(\boldsymbol{v}_{l}^{\varepsilon}\right)\right\|_{L^{\infty}\left(0, T ; L^{4}\left(\Omega_{l}^{\varepsilon}\right)\right)} \leq C_{v} M$, the nonegativity of initial condition $c_{l}^{0}$ and applying Gronwall's inequality we obtain

$$
\left\|c_{a,-}^{\varepsilon}\right\|_{L^{\infty}\left(0, T ; L^{2}\left(\Omega_{a}^{\varepsilon}\right)\right)}+\left\|c_{s,-}^{\varepsilon}\right\|_{L^{\infty}\left(0, T ; L^{2}\left(\Omega_{s}^{\varepsilon}\right)\right)} \leq 0,
$$

which implies the nonnegativity of $c_{l}^{\varepsilon, n}$ a.e. in $(0, T) \times \Omega_{l}^{\varepsilon}$, where $l=a, s$. For $c_{l}^{\varepsilon, n} \in H^{1}\left(0, T ; H^{1}\left(\Omega_{l}^{\varepsilon}\right)\right)$ we obtain also that $c_{l}^{\varepsilon, n} \geq 0$ a.e. on $[0, T] \times \Gamma_{z s}^{\varepsilon}$. Considering weak convergence of $\left\{c_{l}^{\varepsilon, n}\right\}$ in $H^{1}\left(0, T ; H^{1}\left(\Omega_{l}^{\varepsilon}\right)\right)$, as $n \rightarrow \infty$, we conclude that $c_{l}^{\varepsilon}(t, x) \geq 0$ a.e. in $[0, T] \times \Omega_{l}^{\varepsilon}$ and a.e. on $[0, T] \times \Gamma_{z s}^{\varepsilon}$.

Taking $\varphi_{1}=c_{a}^{\varepsilon}$ and $\varphi_{2}=c_{s}^{\varepsilon}$ as test functions in (4.2) and (4.3), using the nonnegativity of $\vartheta_{f, l}^{\varepsilon}$ and of coefficients $\alpha_{l}^{\varepsilon}$, with $l=a, s$, assumptions on $F_{l}$, the first estimate in

$$
\begin{aligned}
& \varepsilon\left\|c_{l}^{\varepsilon}\right\|_{L^{2}\left(\Gamma_{z s}^{\varepsilon}\right)}^{2} \leq C\left(\left\|c_{l}^{\varepsilon}\right\|_{L^{2}\left(\Omega_{l}^{\varepsilon}\right)}^{2}+\varepsilon^{2}\left\|\nabla c_{l}^{\varepsilon}\right\|_{L^{2}\left(\Omega_{l}^{\varepsilon}\right)}^{2}\right), \\
& \varepsilon\left\|c_{l}^{\varepsilon}\right\|_{L^{1}\left(\Gamma_{z s}^{\varepsilon}\right)} \leq C\left(\left\|c_{l}^{\varepsilon}\right\|_{L^{1}\left(\Omega_{l}^{\varepsilon}\right)}+\varepsilon\left\|\nabla c_{l}^{\varepsilon}\right\|_{L^{1}\left(\Omega_{l}^{\varepsilon}\right)}\right),
\end{aligned}
$$

see [27], considering that $\varepsilon\left|\Gamma_{z s}^{\varepsilon}\right| \leq C$ independently of $\varepsilon$, and applying Hölder's inequality, we obtain

$$
\begin{aligned}
\left\|c_{l}^{\varepsilon}(\tau)\right\|_{L^{2}\left(\Omega_{l}^{\varepsilon}\right)}^{2}+\left(d_{l}-\zeta \varepsilon^{2}\right)\left\|\nabla c_{l}^{\varepsilon}\right\|_{L^{2}\left((0, \tau) \Omega_{l}^{\varepsilon}\right)}^{2} & \leq \frac{1}{d_{l}}\left\|H_{M}\left(\boldsymbol{v}_{l}^{\varepsilon}\right)\right\|_{L^{\infty}\left(0, \tau ; L^{4}\left(\Omega_{l}^{\varepsilon}\right)\right)}^{2}\left\|c_{l}^{\varepsilon}\right\|_{L^{2}\left(0, \tau ; L^{4}\left(\Omega_{l}^{\varepsilon}\right)\right)}^{2} \\
& +C_{1}\left\|c_{l}^{\varepsilon}\right\|_{L^{2}\left((0, \tau) \times \Omega_{l}^{\varepsilon}\right)}^{2}+\left\|c_{l}^{0}\right\|_{L^{2}\left(\Omega_{l}^{\varepsilon}\right)}^{2}+C_{2}\left\|\vartheta_{b, l-1}^{\varepsilon}\right\|_{L^{\infty}\left((0, \tau) \times \Gamma_{z s}^{\varepsilon}\right)}^{2}
\end{aligned}
$$

for $\tau \in(0, T]$, with $l=a, s$, whereas $a-1:=s$ and $s-1:=a$. Using the extension of $c_{l}^{\varepsilon}$ from $\Omega_{l}^{\varepsilon}$ into $\Omega$, given by Lemma 4.4, and applying inequality (4.29) in the first term on the right-hand side imply

$$
\begin{aligned}
\| c_{l}^{\varepsilon}(\tau) & \left\|_{L^{2}(\Omega)}^{2}+\left(d_{l} / 4-\zeta \varepsilon^{2}\right)\right\| \nabla c_{l}^{\varepsilon}\left\|_{L^{2}((0, \tau) \times \Omega)}^{2} \leq\right\| c_{l}^{0}\left\|_{L^{2}\left(\Omega_{l}^{\varepsilon}\right)}^{2}+C_{1}\right\| \vartheta_{b, l-1}^{\varepsilon} \|_{L^{\infty}\left((0, T) \times \Gamma_{z s}^{\varepsilon}\right)}^{2} \\
& +C_{2}\left(\left\|H_{M}\left(\boldsymbol{v}_{l}^{\varepsilon}\right)\right\|_{L^{\infty}\left(0, T ; L^{4}\left(\Omega_{l}^{\varepsilon}\right)\right)}^{8}+\left\|H_{M}\left(\boldsymbol{v}_{l}^{\varepsilon}\right)\right\|_{L^{\infty}\left(0, T ; L^{4}\left(\Omega_{l}^{\varepsilon}\right)\right)}^{2}+1\right)\left\|c_{l}^{\varepsilon}\right\|_{L^{2}\left((0, \tau) \times \Omega_{l}^{\varepsilon}\right)}^{2} .
\end{aligned}
$$

Then, for all $\varepsilon \leq \varepsilon_{0}$, with some $\varepsilon_{0}>0$, and $\zeta$ such that $d_{l} / 4-\zeta \varepsilon_{0}^{2} \geq d_{0}>0$, considering the regularity assumption on initial data, the boundedness of $\vartheta_{b, l}^{\varepsilon}$, where $l=a, s$, applying Gronwall's Lemma in inequality (4.31), and using the first inequality in (4.30), we obtain the first estimate in (4.25).

To show $L^{\infty}$-estimates for the extension of $c_{l}^{\varepsilon}$ into $\Omega$, given by Lemma 4.4, we shall apply Theorem II.6.1 from [22] stating that inequality $\left\|\left(c_{l}^{\varepsilon}-S\right)_{+}\right\|_{L^{\infty}\left(0, T ; L^{2}(\Omega)\right)}+\left\|\nabla\left(c_{l}^{\varepsilon}-S\right)_{+}\right\|_{L^{2}\left(\Omega_{T}\right)} \leq \zeta S\left\|\Omega_{l, S}(t)\right\|_{L^{\tilde{q}}(0, T)}^{\tilde{r}}$, for appropriate $\tilde{r}, \tilde{q}$, a positive constant $\zeta$ and $\Omega_{l, S}(t)=\left\{x \in \Omega: c_{l}^{\varepsilon}(t, x)>S\right\}$ for a.a. $t \in(0, T)$, ensures the corresponding estimate for the $L^{\infty}$-norm of $c_{l}^{\varepsilon}$. 
We take $\left(c_{a}^{\varepsilon}-S\right)_{+}$and $\left(c_{s}^{\varepsilon}-S\right)_{+}$as test functions in (4.2) and (4.3). Considering the nonnegativity of $\alpha_{l}^{\varepsilon}(t, x), c_{l}^{\varepsilon}(t, x)$ and $\vartheta_{f, l}^{\varepsilon}(t, x)$ for $(t, x) \in(0, T) \times \Gamma_{z s}^{\varepsilon}$, and inequalities (4.30), the boundary integrals can be estimated by

$$
\begin{gathered}
\varepsilon\left\langle\beta_{l-1}^{\varepsilon}(t, x) \vartheta_{b, l-1}^{\varepsilon}-\alpha_{l}^{\varepsilon}(t, x) c_{l}^{\varepsilon} \vartheta_{f, l}^{\varepsilon},\left(c_{l}^{\varepsilon}-S\right)_{+}\right\rangle_{\Gamma_{z s}^{\varepsilon}} \leq \sup _{\Gamma_{z s}^{\varepsilon}}\left(\beta_{l-1}^{\varepsilon}(t, x) \vartheta_{b, l-1}^{\varepsilon}\right) \varepsilon\left\|\left(c_{l}^{\varepsilon}-S\right)_{+}\right\|_{L^{1}\left(\Gamma_{z s}^{\varepsilon}\right)} \\
\leq 1 / 2\left(\Upsilon_{l}\left\|\left(c_{l}^{\varepsilon}-S\right)_{+}\right\|_{L^{2}\left(\Omega_{l}^{\varepsilon}\right)}^{2}+d_{l}\left\|\nabla\left(c_{l}^{\varepsilon}-S\right)_{+}\right\|_{L^{2}\left(\Omega_{l}^{\varepsilon}\right)}^{2}+C_{Y_{l}}^{2} \Upsilon_{l}\left(1+\Upsilon_{l} \varepsilon^{2} / d_{l}\right)\left|\Omega_{l, S}^{\varepsilon}(t)\right|\right)
\end{gathered}
$$

where $l=a, s$, with $a-1=s, s-1=a, \Omega_{l, S}^{\varepsilon}(t)=\left\{x \in \Omega_{l}^{\varepsilon}: c_{l}^{\varepsilon}(t, x)>S\right\}$ for a.a. $t \in(0, T)$ and $\Upsilon_{l}=\sup _{(0, T) \times \Gamma_{z s}^{\varepsilon}}\left(\beta_{l-1}^{\varepsilon}(t, x) \vartheta_{b, l-1}^{\varepsilon}\right)$. The Lipschitz continuity of $F_{l}$, with $l=a$, s, ensures

$$
\left\langle F_{l}^{\varepsilon}\left(t, x, c_{l}^{\varepsilon}\right),\left(c_{l}^{\varepsilon}-S\right)_{+}\right\rangle_{\Omega_{l}^{\varepsilon}} \leq 3 C_{F_{l}} / 2\left\|\left(c_{l}^{\varepsilon}-S\right)_{+}\right\|_{L^{2}\left(\Omega_{l}^{\varepsilon}\right)}^{2}+\left(S^{2} / C_{F_{l}}+C_{F_{l}}\right)\left|\Omega_{l, S}^{\varepsilon}(t)\right| .
$$

As next we shall estimate $\left|\left\langle H_{M}\left(\boldsymbol{v}_{l}^{\varepsilon}\right) c_{l}^{\varepsilon}, \nabla\left(c_{l}^{\varepsilon}-S\right)_{+}\right\rangle_{\Omega_{l}^{\varepsilon}, T}\right|$ with $l=a, s$. Using Hölder's inequality yields

$$
\begin{aligned}
& \left|\left\langle H_{M}\left(\boldsymbol{v}_{l}^{\varepsilon}\right) c_{l}^{\varepsilon}, \nabla\left(c_{l}^{\varepsilon}-S\right)_{+}\right\rangle_{\Omega_{l}^{\varepsilon}, T}\right| \leq \frac{d_{l}}{4}\left\|\nabla\left(c_{l}^{\varepsilon}-S\right)_{+}\right\|_{L^{2}\left((0, T) \times \Omega_{l}^{\varepsilon}\right)}^{2} \\
& +\frac{2}{d_{l}}\left\|H_{M}\left(\boldsymbol{v}_{l}^{\varepsilon}\right)\right\|_{L^{10}\left(0, T ; L^{4}\left(\Omega_{l}^{\varepsilon}\right)\right)}^{2}\left(\left\|\left(c_{l}^{\varepsilon}-S\right)_{+}\right\|_{L^{5 / 2}\left(0, T ; L^{4}\left(\Omega_{l}^{\varepsilon}\right)\right)}^{2}+S^{2}\left(\int_{0}^{T}\left|\Omega_{l, S}^{\varepsilon}(t)\right|^{\frac{5}{8}} d t\right)^{\frac{4}{5}}\right) .
\end{aligned}
$$

Choosing $\varsigma=1 / 30$ we can estimate

$$
\left\|\left(c_{l}^{\varepsilon}-S\right)_{+}\right\|_{L^{5 / 2}\left(0, T ; L^{4}\left(\Omega_{l}^{\varepsilon}\right)\right)}^{2} \leq\left\|\left(c_{l}^{\varepsilon}-S\right)_{+}\right\|_{L^{5(1+\varsigma) / 2}\left(0, T ; L^{4(1+\varsigma)}\left(\Omega_{l}^{\varepsilon}\right)\right)}^{2}\left(\int_{0}^{T}\left|\Omega_{l, S}^{\varepsilon}(t)\right|^{\frac{5}{8}} d t\right)^{\frac{4}{5} \frac{\varsigma}{\varsigma+1}} .
$$

Using imbedding result, see [22, Chapter II, Eq. (3.4)], and extension of $c_{l}^{\varepsilon}$ into $\Omega$, we obtain

$$
\begin{aligned}
\left\|\left(c_{l}^{\varepsilon}-S\right)_{+}\right\|_{L^{5(1+\varsigma) / 2}\left(0, T ; L^{4(1+\varsigma)}\left(\Omega_{l}^{\varepsilon}\right)\right)}^{2} & \leq\left\|\left(c_{l}^{\varepsilon}-S\right)_{+}\right\|_{L^{5(1+\varsigma) / 2}\left(0, T ; L^{4(1+\varsigma)}(\Omega)\right)}^{2} \\
& \leq C_{\Omega}\left(\left\|\left(c_{l}^{\varepsilon}-S\right)_{+}\right\|_{L^{\infty}\left(0, T ; L^{2}(\Omega)\right)}^{2}+\left\|\nabla\left(c_{l}^{\varepsilon}-S\right)_{+}\right\|_{L^{2}\left(0, T ; L^{2}(\Omega)\right)}^{2}\right) .
\end{aligned}
$$

Considering $\left\|H_{M}\left(\boldsymbol{v}_{l}^{\varepsilon}\right)\right\|_{L^{10}\left(0, T ; L^{4}\left(\Omega_{l}^{\varepsilon}\right)\right)}^{2} \leq T^{\frac{1}{5}}\left\|H_{M}\left(\boldsymbol{v}_{l}^{\varepsilon}\right)\right\|_{L^{\infty}\left(0, T ; L^{4}\left(\Omega_{l}^{\varepsilon}\right)\right)}^{2} \leq T^{\frac{1}{5}} C_{v} M^{2}$ we conclude

$$
\begin{gathered}
\left|\left\langle H_{M}\left(\boldsymbol{v}_{l}^{\varepsilon}\right) c_{l}^{\varepsilon}, \nabla\left(c_{l}^{\varepsilon}-S\right)_{+}\right\rangle_{\Omega_{l}^{\varepsilon}, T}\right| \leq \frac{d_{l}}{4}\left\|\nabla\left(c_{l}^{\varepsilon}-S\right)_{+}\right\|_{L^{2}\left((0, T) \times \Omega_{l}^{\varepsilon}\right)}^{2}+\frac{2}{d_{l}} T^{\frac{1}{5}} C_{\Omega} C_{v} M^{2} \times \\
\quad\left[S^{2}\left(\int_{0}^{T}\left|\Omega_{l, S}^{\varepsilon}\right|^{\frac{5}{8}} d t\right)^{\frac{4}{5}}+\left(\int_{0}^{T}\left|\Omega_{l, S}^{\varepsilon}\right|^{\frac{5}{8}} d t\right)^{\frac{4}{5} \frac{\varsigma}{\varsigma+1}}\left(\left\|\left(c_{l}^{\varepsilon}-S\right)_{+}\right\|_{L^{\infty}\left(0, T ; L^{2}(\Omega)\right)}^{2}+\left\|\nabla\left(c_{l}^{\varepsilon}-S\right)_{+}\right\|_{L^{2}\left(\Omega_{T}\right)}^{2}\right)\right] .
\end{gathered}
$$

We shall define

$$
S=\max _{l=a, s} \max \left\{1,\left\|c_{l}^{0}\right\|_{L^{\infty}(\Omega)}, \Xi\left(\left\|c_{l}^{0}\right\|_{L^{1}(\Omega)}+\Upsilon_{l}|\Gamma||\Omega|+C_{F_{l}}|\Omega|\right) e^{C_{F_{l}} T}, C_{F_{l}}, \Upsilon_{l}\right\},
$$

where $\Xi=\Xi\left(Y, Y_{l}\right)$ is the constant from Lemma 4.4. The choice of $S$ ensures that $\left|\Omega_{l, S}^{\varepsilon}(t)\right| \leq 1$ and $\left|\Omega_{l, S}(t)\right| \leq 1$. Choosing

$\tilde{T}=\min _{l=a, s} \min \left\{\left[d_{l}^{2} /\left(16 \Xi C_{\Omega} C_{v} M^{2}|\Omega|^{\frac{\varsigma}{2 \varsigma+2}}\right)\right]^{\frac{5(\varsigma+1)}{5 \varsigma+1}},\left[d_{l} /\left(8 \Xi C_{\Omega} C_{v} M^{2}|\Omega|^{\frac{\varsigma}{2 \varsigma+2}}\right)\right]^{\frac{5(\varsigma+1)}{5 \varsigma+1}},\left[4 \Upsilon_{l}+12 C_{F_{l}}\right]^{-1},|\Omega|^{-\frac{5}{8}}\right\}$, combining estimates (4.32)-(4.34) and using the fact that $\left|\Omega_{l, S}^{\varepsilon}(t)\right| \leq 1$ yield

$$
\begin{aligned}
& \left\|\left(c_{l}^{\varepsilon}-S\right)_{+}\right\|_{L^{\infty}\left(0, \tilde{T} ; L^{2}(\Omega)\right)}^{2}+\left\|\nabla\left(c_{l}^{\varepsilon}-S\right)_{+}\right\|_{L^{2}((0, \tilde{T}) \times \Omega)}^{2} \\
& \quad \leq S^{2} 8 \Xi / \min \left\{1, d_{l}\right\}\left(2 \tilde{T}^{\frac{1}{5}} C_{v} C_{\Omega} M^{2} / d_{l}+1 / C_{F_{l}}+1+C_{Y_{l}}^{2}\left(1+\Upsilon_{l} \varepsilon^{2} / d_{l}\right) / 2\right)\left(\int_{0}^{\tilde{T}}\left|\Omega_{l, S}^{\varepsilon}(t)\right|^{\frac{5}{8}} d t\right)^{\frac{4}{5}} .
\end{aligned}
$$


Choosing $q=4(1+\varsigma)$ together with $r=5(1+\varsigma) / 2$ and using $\left|\Omega_{l, S}^{\varepsilon}(t)\right| \leq\left|\Omega_{l, S}(t)\right| \leq 1$, we obtain

$$
\left\|\left(c_{l}^{\varepsilon}-S\right)_{+}\right\|_{L^{\infty}\left(0, \tilde{T} ; L^{2}(\Omega)\right)}+\left\|\nabla\left(c_{l}^{\varepsilon}-S\right)_{+}\right\|_{L^{2}((0, \tilde{T}) \times \Omega)} \leq S \Theta\left(\int_{0}^{\tilde{T}}\left|\Omega_{l, S}(t)\right|^{\frac{r}{q}} d t\right)^{\frac{2(1+\varsigma)}{r}}
$$

where $\Theta=8 \Xi / \min \left\{1, d_{l}\right\}\left(2 \tilde{T}^{\frac{1}{5}} C_{v} C_{\Omega} M^{2} / d_{l}+1 / C_{F_{l}}+1+C_{Y_{l}}^{2}\left(1+\Upsilon_{l} \varepsilon^{2} / d_{l}\right) / 2\right)$. Applying Theorem II.6.1 in [22] yields

$$
\left\|c_{l}^{\varepsilon}\right\|_{L^{\infty}((0, \tilde{T}) \times \Omega)} \leq 2 S\left[1+2^{\frac{2 \varsigma+1}{\varsigma^{2}}}\left(C_{\Omega} \Theta\right)^{1+\frac{1}{\varsigma}} \tilde{T}^{1+\frac{1}{\varsigma}}|\Omega|^{\frac{1}{4}}\right] .
$$

Considering $T_{c}=\min _{l=a, s} \min \left\{\tilde{T}, \frac{d_{l}^{5}}{\left(2 C_{\Omega} C_{v} M^{2}\right)^{5}},|\Omega|^{-\frac{\varsigma}{4(1+\varsigma)}}\left[\frac{8 C_{\Omega} \Xi}{\min \left\{1, d_{l}\right\}}\left[2+\frac{1}{C_{F_{l}}}+\frac{C_{Y_{l}}^{2}}{2}\left(1+\Upsilon_{l} \frac{\varepsilon^{2}}{d_{l}}\right)\right] 2^{\frac{2+\varsigma}{\varsigma+\varsigma^{2}}}\right]^{-1}\right\}$ implies

$$
\left\|c_{l}^{\varepsilon}\right\|_{L^{\infty}((0, \tau) \times \Omega)} \leq 4 S \quad \text { for all } \tau \leq T_{c} .
$$

The iteration over time-intervals will then ensure the boundedness of $c_{l}^{\varepsilon}$ in $(0, T) \times \Omega$, and thus also the boundedness of $c_{l}^{\varepsilon}$ in $(0, T) \times \Omega_{l}^{\varepsilon}$, for $l=a, s$, and the second estimate in (4.25).

Using the estimates for $c_{l}^{\varepsilon}$ and $\vartheta_{j, l}^{\varepsilon}$, with $j=f, b$ and $l=a, s$, and considering $\partial_{t} \vartheta_{j, l}^{\varepsilon}$ as test functions in (3.5), we conclude

$$
\varepsilon^{1 / 2}\left\|\partial_{t} \vartheta_{f, l}^{\varepsilon}\right\|_{L^{2}\left((0, T) \times \Gamma_{z s}^{\varepsilon}\right)}+\varepsilon^{1 / 2}\left\|\partial_{t} \vartheta_{b, l}^{\varepsilon}\right\|_{L^{2}\left((0, T) \times \Gamma_{z s}^{\varepsilon}\right)} \leq C_{1}\left(1+\varepsilon^{1 / 2}\left\|c_{l}^{\varepsilon}\right\|_{L^{2}\left((0, T) \times \Gamma_{z s}^{\varepsilon}\right)}\right) \leq C_{2} .
$$

Differentiating equations (3.1) and boundary conditions (3.8), (3.10) with respect to $t$, considering $\left(\partial_{t} \boldsymbol{v}_{z}^{\varepsilon}, \partial_{t} \boldsymbol{v}_{a}^{\varepsilon}, \partial_{t} \boldsymbol{v}_{s p}^{\varepsilon}\right)$ as a test function, and applying Korn inequality (4.4) we obtain for $\tau \in(0, T]$

$$
\begin{gathered}
\varepsilon^{2}\left\|\nabla \partial_{t} \boldsymbol{v}_{z}^{\varepsilon}\right\|_{L^{2}\left((0, \tau) \times \Omega_{z}^{\varepsilon}\right)}^{2}+\left\|\partial_{t} \boldsymbol{v}_{s}^{\varepsilon}\right\|_{L^{2}\left((0, \tau) \times \Omega_{s}^{\varepsilon}\right)}^{2}+\left\|\partial_{t} \boldsymbol{v}_{a}^{\varepsilon}\right\|_{L^{2}\left((0, \tau) \times \Omega_{a}^{\varepsilon}\right)}^{2}+\varepsilon\left\|\partial_{t} \boldsymbol{v}_{a}^{\varepsilon} \cdot \boldsymbol{n}\right\|_{L^{2}\left((0, \tau) \times \Gamma_{z s}^{\varepsilon}\right)}^{2} \\
\leq C \varepsilon\left(\left\|\partial_{t} c_{s}^{\varepsilon}\right\|_{L^{2}\left((0, \tau) \times \Gamma_{z s}^{\varepsilon}\right)}^{2}+\left\|\partial_{t} c_{a}^{\varepsilon}\right\|_{L^{2}\left((0, \tau) \times \Gamma_{z s}^{\varepsilon}\right)}^{2}\right) .
\end{gathered}
$$

Now we differentiate with respect to $t$ equations (3.2) and use $\partial_{t} c_{a}^{\varepsilon}$ and $\partial_{t} c_{s}^{\varepsilon}$ as test functions. Estimates (4.25) together with inequalities (4.29) and (4.30), estimates in Lemma 4.4, and Assumption 1 give

$$
\begin{aligned}
& \left\|\partial_{t} c_{l}^{\varepsilon}(\tau)\right\|_{L^{2}\left(\Omega_{l}^{\varepsilon}\right)}^{2}+\int_{0}^{\tau}\left\|\partial_{t} \nabla c_{l}^{\varepsilon}\right\|_{L^{2}\left(\Omega_{l}^{\varepsilon}\right)}^{2} d t \leq C\left\|c_{l}^{\varepsilon}\right\|_{L^{\infty}\left((0, T) \times \Omega_{l}^{\varepsilon}\right)}^{2} \int_{0}^{\tau}\left\|\partial_{t} \boldsymbol{v}_{l}^{\varepsilon}\right\|_{L^{2}\left(\Omega_{l}^{\varepsilon}\right)}^{2} d t+\left\|\partial_{t} c_{l}^{\varepsilon}(0)\right\|_{L^{2}\left(\Omega_{l}^{\varepsilon}\right)}^{2} \\
& +C\left(1+\left\|H_{M}\left(\boldsymbol{v}_{l}^{\varepsilon}\right)\right\|_{L^{\infty}\left(0, \tau ; L^{4}\left(\Omega_{l}^{\varepsilon}\right)\right)}^{2}\right) \int_{0}^{\tau}\left[\left\|\partial_{t} c_{l}^{\varepsilon}\right\|_{L^{2}\left(\Omega_{l}^{\varepsilon}\right)}^{2}+\varepsilon\left\|\partial_{t} \vartheta_{b, l-1}^{\varepsilon}\right\|_{L^{2}\left(\Gamma_{z s}^{\varepsilon}\right)}^{2}+\varepsilon\left\|\partial_{t} \vartheta_{f, l}^{\varepsilon}\right\|_{L^{2}\left(\Gamma_{z s}^{\varepsilon}\right)}^{2}\right] d t,
\end{aligned}
$$

where $l=a, s$ with $a-1:=s$ and $s-1:=a$. The regularity assumption on $c_{l}^{0}$ ensures that $\left\|\partial_{t} c_{l}^{\varepsilon}(0)\right\|_{L^{2}\left(\Omega_{l}^{\varepsilon}\right)} \leq C\left\|c_{l}^{0}\right\|_{H^{2}\left(\Omega_{l}^{\varepsilon}\right)}$, with $l=a, s$. Combining the last inequality together with (4.36) and (4.37), using the boundedness of $H_{M}\left(\boldsymbol{v}_{l}^{\varepsilon}\right)$ and inequality (4.30), choosing $\varepsilon$ sufficient small, and applying Gronwall's inequality imply (4.26).

Uniqueness. Suppose there are two solutions of the problem. We denote $c_{l}^{\varepsilon}=c_{l, 1}^{\varepsilon}-c_{l, 2}^{\varepsilon}$ and $\vartheta_{j, l}^{\varepsilon}=$ $\vartheta_{j, l, 1}^{\varepsilon}-\vartheta_{j, l, 2}^{\varepsilon}$, with $j=f, b$ and $l=a, s$, and choose $\varphi_{1}=c_{a}^{\varepsilon}$ in (4.2) and $\varphi_{2}=c_{s}^{\varepsilon}$ in (4.3)

$$
\begin{aligned}
& \left\|c_{l}^{\varepsilon}(\tau)\right\|_{L^{2}\left(\Omega_{l}^{\varepsilon}\right)}^{2}+2\left(d_{l}-\zeta_{1}\right) \int_{0}^{\tau}\left\|\nabla c_{l}^{\varepsilon}\right\|_{L^{2}\left(\Omega_{l}^{\varepsilon}\right)} d t \leq C_{\zeta_{1}}\left\|c_{l, 1}^{\varepsilon}\right\|_{L^{\infty}\left((0, T) \times \Omega_{l}^{\varepsilon}\right)}^{2} \int_{0}^{\tau}\left\|\boldsymbol{v}_{l, 1}^{\varepsilon}-\boldsymbol{v}_{l, 2}^{\varepsilon}\right\|_{L^{2}\left(\Omega_{l}^{\varepsilon}\right)}^{2} d t \\
& +C_{\zeta_{1}}\left(1+\left\|H_{M}\left(\boldsymbol{v}_{l, 2}^{\varepsilon}\right)\right\|_{L^{\infty}\left(0, \tau ; L^{4}\left(\Omega_{l}^{\varepsilon}\right)\right)}^{2}\right) \int_{0}^{\tau}\left\|c_{l}^{\varepsilon}\right\|_{L^{2}\left(\Omega_{l}^{\varepsilon}\right)}^{2} d t+C_{\zeta_{2}}\left\|c_{l, 1}^{\varepsilon}\right\|_{L^{\infty}\left((0, T) \times \Gamma_{z s}^{\varepsilon}\right)}^{2} \int_{0}^{\tau} \varepsilon\left\|\vartheta_{f, l}^{\varepsilon}\right\|_{L^{2}\left(\Gamma_{z s}^{\varepsilon}\right)}^{2} d t \\
& +C_{\zeta_{2}} \int_{0}^{\tau} \varepsilon\left\|\vartheta_{b, l-1}^{\varepsilon}\right\|_{L^{2}\left(\Gamma_{z s}^{\varepsilon}\right)}^{2} d t+\zeta_{2} \int_{0}^{\tau} \varepsilon\left\|c_{l}^{\varepsilon}\right\|_{L^{2}\left(\Gamma_{z s}^{\varepsilon}\right)}^{2} d t,
\end{aligned}
$$


for any $\tau \in(0, T]$, where $a-1:=s$ and $s-1:=a$. For $\vartheta_{j, l}^{\varepsilon}$, with $j=f, b$ and $l=a, s$, we obtain

$$
\begin{aligned}
& \partial_{t}\left\|\vartheta_{f, l}^{\varepsilon}\right\|_{L^{2}\left(\Gamma_{z s}^{\varepsilon}\right)}^{2} \leq C\left[\left(1+\left\|c_{l, 1}^{\varepsilon}\right\|_{L^{\infty}}\right)\left\|\vartheta_{f, l}^{\varepsilon}\right\|_{L^{2}\left(\Gamma_{z s}^{\varepsilon}\right)}^{2}+\left\|\vartheta_{f, l, 2}^{\varepsilon}\right\|_{L^{\infty}}^{2}\left\|c_{l}^{\varepsilon}\right\|_{L^{2}\left(\Gamma_{z s}^{\varepsilon}\right)}^{2}+\left\|\vartheta_{b, l}^{\varepsilon}\right\|_{L^{2}\left(\Gamma_{z s}^{\varepsilon}\right)}^{2}\right], \\
& \partial_{t}\left\|\vartheta_{b, l}^{\varepsilon}\right\|_{L^{2}\left(\Gamma_{z s}^{\varepsilon}\right)}^{2} \leq C\left[\left\|c_{l, 1}^{\varepsilon}\right\|_{L^{\infty}}^{2}\left\|\vartheta_{f, l}^{\varepsilon}\right\|_{L^{2}\left(\Gamma_{z s}^{\varepsilon}\right)}^{2}+\left\|\vartheta_{f, l, 2}^{\varepsilon}\right\|_{L^{\infty}}^{2}\left\|c_{l}^{\varepsilon}\right\|_{L^{2}\left(\Gamma_{z s}^{\varepsilon}\right)}^{2}+\left\|\vartheta_{b, l}^{\varepsilon}\right\|_{L^{2}\left(\Gamma_{z s}^{\varepsilon}\right)}^{2}\right] .
\end{aligned}
$$

Combining those estimates, using (4.14) and (4.30), boundedness of $c_{l}^{\varepsilon}$ and $H_{M}\left(\boldsymbol{v}_{l}^{\varepsilon}\right)$, and applying Gronwall's inequality yield

$$
\varepsilon\left\|\vartheta_{j, l}^{\varepsilon}\right\|_{L^{\infty}\left(0, T ; L^{2}\left(\Gamma_{z s}^{\varepsilon}\right)\right)}^{2}+\left\|c_{l}^{\varepsilon}\right\|_{L^{\infty}\left(0, T ; L^{2}\left(\Omega_{l}^{\varepsilon}\right)\right)}^{2}+\left\|\boldsymbol{v}_{l, 1}^{\varepsilon}-\boldsymbol{v}_{l, 2}^{\varepsilon}\right\|_{L^{2}\left((0, T) \times \Omega_{l}^{\varepsilon}\right)}^{2} \leq 0 .
$$

Thus, $c_{l, 1}^{\varepsilon}=c_{l, 2}^{\varepsilon}, \boldsymbol{v}_{l, 1}^{\varepsilon}=\boldsymbol{v}_{l, 2}^{\varepsilon}$ a.e. in $(0, T) \times \Omega_{l}^{\varepsilon}$, and $\vartheta_{j, l, 1}^{\varepsilon}=\vartheta_{j, l, 2}^{\varepsilon}$ a.e. on $[0, T] \times \Gamma_{z s}^{\varepsilon}$, with $l=a, s$ and $j=f, b$.

\section{Derivation of macroscopic equations}

We denote by $\tilde{\boldsymbol{v}}_{i}^{\varepsilon}$, for $i=z, a, s p$ the extension by zero from $(0, T) \times \Omega_{i}^{\varepsilon}$ into $\Omega_{T}=(0, T) \times \Omega$, where $\Omega_{s p}^{\varepsilon}:=\Omega_{a s}^{\varepsilon}$, and define $\boldsymbol{v}^{\varepsilon}=\tilde{\boldsymbol{v}}_{z}^{\varepsilon}+\tilde{\boldsymbol{v}}_{a}^{\varepsilon}+\tilde{\boldsymbol{v}}_{s p}^{\varepsilon}$ in $\Omega_{T}$.

Lemma 5.1. Under Assumption 1 there exist $\boldsymbol{v}_{z} \in L^{2}\left(\Omega_{T} ; H_{p e r}^{1}\left(Y_{z}\right) / \mathbb{R}\right), \boldsymbol{v}_{a} \in L^{2}\left(\Omega_{T} \times Y_{a}\right), \boldsymbol{v}_{s p} \in$ $L^{2}\left(\Omega_{T} \times Y_{a s}\right)$ and $p_{a}, p_{s} \in L^{2}\left(\Omega_{T} \times Y\right)$, with $\left\langle p_{a}(t, x, y), 1\right\rangle_{\Omega \times Y_{a}}+\left\langle p_{s}(t, x, y), 1\right\rangle_{\Omega \times Y_{s}}=0 \quad$ for a.a. $t \in(0, T)$, such that, up to a subsequence,

$$
\begin{cases}\boldsymbol{v}_{z}^{\varepsilon} \rightarrow \boldsymbol{v}_{z}, \quad \boldsymbol{v}_{a}^{\varepsilon} \rightarrow \boldsymbol{v}_{a}, \quad \boldsymbol{v}_{s p}^{\varepsilon} \rightarrow \boldsymbol{v}_{s p} & \text { two-scale, } \\ \boldsymbol{v}_{s}^{\varepsilon} \rightarrow \tilde{\boldsymbol{v}}_{z}+\tilde{\boldsymbol{v}}_{s p} & \text { two-scale in } \Omega_{T} \times Y_{s}, \\ \boldsymbol{v}^{\varepsilon} \rightarrow \overline{\boldsymbol{v}}=\tilde{\boldsymbol{v}}_{z}+\tilde{\boldsymbol{v}}_{a}+\tilde{\boldsymbol{v}}_{s p} & \text { two-scale in } \Omega_{T} \times Y, \\ \varepsilon \nabla \boldsymbol{v}_{z}^{\varepsilon} \rightarrow \nabla_{y} \boldsymbol{v}_{z} & \text { two-scale in } \Omega_{T} \times Y_{z}, \\ P_{a}^{\varepsilon} \rightarrow p_{a}, P_{s}^{\varepsilon} \rightarrow p_{s} & \text { two-scale in } \Omega_{T} \times Y, \\ \left.p_{a}^{\varepsilon} \rightarrow p_{a}\right|_{\Omega_{T} \times Y_{a}} \text { two-scale in } \Omega_{T} \times Y_{a}, & \left.p_{s}^{\varepsilon} \rightarrow p_{s}\right|_{\Omega_{T} \times Y_{s}} \text { two-scale in } \Omega_{T} \times Y_{s},\end{cases}
$$

and

$$
\begin{cases}P_{a}^{\varepsilon} \rightarrow \frac{1}{|Y|} \int_{Y} p_{a} d y, \quad P_{s}^{\varepsilon} \rightarrow \frac{1}{|Y|} \int_{Y} p_{s} d y & \text { weakly in } L^{2}\left(\Omega_{T}\right) \\ \boldsymbol{v}^{\varepsilon} \rightarrow \boldsymbol{v}=\frac{1}{|Y|} \int_{Y_{z}} \boldsymbol{v}_{z} d y+\frac{1}{|Y|} \int_{Y_{a}} \boldsymbol{v}_{a} d y+\frac{1}{|Y|} \int_{Y_{a s}} \boldsymbol{v}_{s p} d y & \text { weakly in } L^{2}\left(\Omega_{T}\right) \\ \boldsymbol{v}_{a}^{\varepsilon} \cdot \boldsymbol{n} \rightarrow \boldsymbol{v}_{a} \cdot \boldsymbol{n} & \text { two-scale on } \Omega_{T} \times \Gamma_{z s}\end{cases}
$$

Proof. The convergences in (5.1) follows directly from estimates (4.12), (4.13) and (4.25) together with Lemma 4.1 in [4] and the definition and compactness theorems for two-scale convergence, see [2, 29] or Definition 5.5 and Theorem 5.7 in Appendix. Since $\tilde{\boldsymbol{v}}_{i}^{\varepsilon}$, for $i=z, a, s p$, are zero in $\Omega \backslash \Omega_{i}^{\varepsilon}$, also the two-scale limits are equal to zero in $Y \backslash Y_{i}$, respectively. Applying two-scale limit in equality $\left\langle p_{a}^{\varepsilon}, 1\right\rangle_{\Omega_{a}^{\varepsilon}}+\left\langle p_{s}^{\varepsilon}, 1\right\rangle_{\Omega_{s}^{\varepsilon}}=0$ from the definition of the space $\mathcal{P}^{\varepsilon}$ we obtain the stated relation for mean values of $p_{a}$ and $p_{s}$. A priori estimates (4.12), (4.13) and the relation between two-scale and weak limits, [2,4,29], ensure the first two convergences in (5.2). The uniform in $\varepsilon$ boundedness of $\varepsilon^{1 / 2}\left\|\boldsymbol{v}_{a}^{\varepsilon} \cdot \boldsymbol{n}\right\|_{L^{2}\left((0, T) \times \Gamma_{z s}^{\varepsilon}\right)}$ implies that there exists $w \in L^{2}\left(\Omega_{T} \times \Gamma_{z s}\right)$ such that $\boldsymbol{v}_{a}^{\varepsilon} \cdot \boldsymbol{n} \rightarrow w$ two-scale, see [28] or Definition 5.8 and Theorem 5.9 in Appendix. Then $\operatorname{div} \boldsymbol{v}_{a}^{\varepsilon}=0$ in $(0, T) \times \Omega_{a}^{\varepsilon}$ and two-scale convergence of $\boldsymbol{v}_{a}^{\varepsilon}$ to $\boldsymbol{v}_{a}$ in $\Omega_{T} \times Y_{a}$ ensure $w=\boldsymbol{v}_{a} \cdot \boldsymbol{n}$ a.e. on $\Omega_{T} \times \Gamma_{z s}$.

We consider the extension of $c_{l}^{\varepsilon}$ from $\Omega_{l}^{\varepsilon}$ into $\Omega$, for $l=a, s$, as in Lemma 4.4, and identify $c_{l}^{\varepsilon}$ with its extension. We denote by $\mathcal{T}_{\Gamma_{z s}}^{\varepsilon}: \Gamma_{z s}^{\varepsilon} \rightarrow \Omega \times \Gamma_{z s}$ the boundary unfolding operator, see [10] or Definition 5.10 in Appendix. Here we shall use a shorted notation $\mathcal{T}_{\Gamma}^{\varepsilon}(\psi):=\mathcal{T}_{\Gamma_{z s}}^{\varepsilon}(\psi)$. 
Lemma 5.2. Under Assumption 1 there exist functions $c_{l} \in H^{1}\left(0, T ; H^{1}(\Omega)\right) \cap L^{\infty}\left(\Omega_{T}\right), c_{l}^{1} \in$ $L^{2}\left(\Omega_{T} ; H_{p e r}^{1}\left(Y_{l}\right) / \mathbb{R}\right)$ and $\vartheta_{j, l} \in L^{\infty}\left(\Omega_{T} \times \Gamma_{z s}\right) \cap H^{1}\left(0, T ; L^{2}\left(\Omega \times \Gamma_{z s}\right)\right)$, with $j=f, b$ and $l=a, s$, such that, up to a subsequence,

$$
\begin{array}{cl}
c_{l}^{\varepsilon} \rightarrow c_{l} \text { weakly in } H^{1}\left(0, T ; H^{1}(\Omega)\right), & \text { weakly-* in } L^{\infty}\left(\Omega_{T}\right), \\
c_{l}^{\varepsilon} \rightarrow c_{l} \text { strongly in } L^{2}\left(0, T ; H^{\varsigma}(\Omega)\right), \varsigma<1, & \varepsilon^{1 / 2}\left\|c_{l}^{\varepsilon}-c_{l}\right\|_{L^{2}\left((0, T) \times \Gamma_{z s}^{\varepsilon}\right)} \rightarrow 0, \\
c_{l}^{\varepsilon} \rightarrow c_{l}, \partial_{t} c_{l}^{\varepsilon} \rightarrow \partial_{t} c_{l}, \nabla c_{l}^{\varepsilon} \rightarrow \nabla c_{l}+\nabla_{y} c_{l}^{1} & \text { two-scale, } \\
\vartheta_{j, l}^{\varepsilon} \rightarrow \vartheta_{j, l}, \partial_{t} \vartheta_{j, l}^{\varepsilon} \rightarrow \partial_{t} \vartheta_{j, l} & \text { two-scale, } \\
\mathcal{T}_{\Gamma}^{\varepsilon} c_{l}^{\varepsilon} \rightarrow c_{l}, \mathcal{T}_{\Gamma}^{\varepsilon} \vartheta_{j, l}^{\varepsilon} \rightarrow \vartheta_{j, l} & \text { strongly in } L^{2}\left(\Omega_{T} \times \Gamma_{z s}\right) .
\end{array}
$$

Proof. The first two convergences are a direct consequence of the a priori estimates for $c_{l}^{\varepsilon}$ in (4.25)-(4.26) and properties of the extension from $\Omega_{l}^{\varepsilon}$ into $\Omega$, with $l=a, s$.

The compact embedding $H^{1}\left(0, T ; H^{1}(\Omega)\right) \subset L^{2}\left(0, T ; H^{\varsigma}(\Omega)\right)$ for $\varsigma<1$ ensures the strong convergence of $c_{a}^{\varepsilon}$ and $c_{s}^{\varepsilon}$. Then, the strong convergence in $L^{2}\left(0, T ; H^{\varsigma}(\Omega)\right)$, with $1 / 2<\varsigma<1$, and the estimate

$$
\varepsilon^{1 / 2}\left\|c_{l}^{\varepsilon}\right\|_{L^{2}\left((0, T) \times \Gamma_{z s}^{\varepsilon}\right)} \leq C\left\|c_{l}^{\varepsilon}\right\|_{L^{2}\left(0, T ; H^{\varsigma}\left(\Omega_{l}^{\varepsilon}\right)\right)}, \quad \text { with } l=a, s,
$$

see [27] for the proof, give the fourth convergence in (5.3). A priori estimates (4.25), (4.26) and compactness theorems for two-scale convergence, see $[2,28,29]$ or Theorem 5.7 and 5.9 in Appendix, imply two-scale convergences stated in (5.3). Theorem 5.9 in Appendix ensures also that $\vartheta_{j, l} \in L^{\infty}\left(\Omega_{T} \times \Gamma_{z s}\right)$.

The assumed structure of the initial data $\vartheta_{j, l}^{0, \varepsilon}$, i.e. $\mathcal{T}_{\Gamma}^{\varepsilon}\left(\vartheta_{j, l}^{0, \varepsilon}\right)(x, y)=\vartheta_{2 j, l}^{0}(y) \mathcal{T}_{\Gamma}^{\varepsilon}\left(\vartheta_{1 j, l}^{0}(x)\right)$ and the strong convergence in $L^{2}\left(\Omega \times \Gamma_{z s}\right)$ of $\mathcal{T}_{\Gamma}^{\varepsilon}(\psi)$ for $\psi \in L^{2}(\Omega)$, see [10], yield $\mathcal{T}_{\Gamma}^{\varepsilon}\left(\vartheta_{j, l}^{0, \varepsilon}\right) \rightarrow \vartheta_{j, l}^{0}$ strongly in $L^{2}\left(\Omega \times \Gamma_{z s}\right)$, with $j=f, b$ and $l=a, s$. The properties of the unfolding operator, see [10], give

$$
\left\|\mathcal{T}_{\Gamma}^{\varepsilon}\left(c_{l}^{\varepsilon}\right)-\mathcal{T}_{\Gamma}^{\varepsilon}\left(c_{l}\right)\right\|_{L^{2}\left(\Omega_{T} \times \Gamma_{z s}\right)} \leq C \varepsilon^{1 / 2}\left\|c_{l}^{\varepsilon}-c_{l}\right\|_{L^{2}\left((0, T) \times \Gamma_{z s}^{\varepsilon}\right)} \quad \text { for } l=a, s,
$$

and $\mathcal{T}_{\Gamma}^{\varepsilon}\left(c_{l}\right) \rightarrow c_{l}$ strongly in $L^{2}\left(\Omega_{T} \times \Gamma_{z s}\right)$. Then, together with the fourth convergence in (5.3) we obtain that $\mathcal{T}_{\Gamma}^{\varepsilon}\left(c_{l}^{\varepsilon}\right) \rightarrow c_{l}$ strongly in $L^{2}\left(\Omega_{T} \times \Gamma_{z s}\right)$, where $l=a, s$. Applying now the unfolding operator to the equations in (3.5), using the convergence of $c_{a}^{\varepsilon}, c_{s}^{\varepsilon}$, and the equivalence between the two-scale convergence of a sequence and the weak convergence of the corresponding unfolded sequence, see [27] or Lemma 5.11 in Appendix, we can show, in the same manner as in [27], that $\left\{\mathcal{T}_{\Gamma}^{\varepsilon}\left(\vartheta_{j, l}^{\varepsilon}\right)\right\}$, with $j=f, b$ and $l=a$, s, are Cauchy sequences in $L^{2}\left(\Omega_{T} \times \Gamma_{z s}\right)$ and conclude the last strong convergences stated in (5.3).

Theorem 5.3. For the sequence of solutions $\left(\boldsymbol{v}_{z}^{\varepsilon}, \boldsymbol{v}_{a}^{\varepsilon}, \boldsymbol{v}_{s p}^{\varepsilon}\right)$ and $\left(p_{z}^{\varepsilon}, p_{a}^{\varepsilon}, p_{s p}^{\varepsilon}\right)$ of microscopic problem (3.1), (3.8), (3.10), we have $\boldsymbol{v}^{\varepsilon} \rightarrow \boldsymbol{v}$ weakly in $L^{2}(0, T ; H(\operatorname{div}, \Omega))$, with $\boldsymbol{v}^{\varepsilon}=\tilde{\boldsymbol{v}}_{z}^{\varepsilon}+\tilde{\boldsymbol{v}}_{s p}^{\varepsilon}+\tilde{\boldsymbol{v}}_{a}^{\varepsilon}$, extensions $P_{s}^{\varepsilon} \rightarrow p$ and $P_{a}^{\varepsilon} \rightarrow p$ weakly in $L^{2}\left(0, T ; L^{2}(\Omega) / \mathbb{R}\right)$ as $\varepsilon \rightarrow 0$, and $(\boldsymbol{v}, p) \in L^{2}(0, T ; H(\operatorname{div}, \Omega)) \times L^{2}\left(0, T ; L_{0}^{2}(\Omega)\right)$, where $L_{0}^{2}(\Omega)=\left\{\phi \in L^{2}(\Omega):\langle\phi, 1\rangle_{\Omega}=0\right\}$, is the unique solution of the Darcy problem

$$
\begin{cases}\boldsymbol{v}+\boldsymbol{K} \nabla p=\boldsymbol{M}\left(c_{s}-c_{a}\right) & \text { in }(0, T) \times \Omega, \\ \operatorname{div} \boldsymbol{v}=0 & \text { in }(0, T) \times \Omega, \\ \boldsymbol{v} \cdot \boldsymbol{n}=v_{D} & \text { on }(0, T) \times \partial \Omega,\end{cases}
$$

where the tensor $\boldsymbol{K}$ and vector $\boldsymbol{M}$ are defined in (5.13), and $c_{s}, c_{a}$ are solutions of the macroscopic equations (5.14)-(5.16).

Proof. The weak convergences of $\boldsymbol{v}^{\varepsilon}$ in $L^{2}\left(\Omega_{T}\right)$ and of $P_{l}^{\varepsilon}$ in $L^{2}\left(0, T ; L^{2}(\Omega) / \mathbb{R}\right)$, with $l=a, s$, follows from Lemma 5.1. Using $\operatorname{div} \boldsymbol{v}^{\varepsilon} \in L^{2}\left(\Omega_{T}\right)$ and $\operatorname{div} \boldsymbol{v}^{\varepsilon}=0$ in $\Omega_{T}$, and applying the weak convergence of $\boldsymbol{v}^{\varepsilon}$ we obtain for $\psi \in C_{0}^{\infty}\left(\Omega_{T}\right)$

$$
0=\lim _{\varepsilon \rightarrow 0}\left\langle\operatorname{div} \boldsymbol{v}^{\varepsilon}, \psi\right\rangle_{\Omega, T}=\lim _{\varepsilon \rightarrow 0}\left\langle\boldsymbol{v}^{\varepsilon}, \nabla \psi\right\rangle_{\Omega, T}=\langle\boldsymbol{v}, \nabla \psi\rangle_{\Omega, T}=\langle\operatorname{div} \boldsymbol{v}, \psi\rangle_{\Omega, T} .
$$


For $\psi \in C^{\infty}\left(\Omega_{T}\right)$ we have

$$
0=\lim _{\varepsilon \rightarrow 0}\left\langle\operatorname{div} \boldsymbol{v}^{\varepsilon}, \psi\right\rangle_{\Omega, T}=\lim _{\varepsilon \rightarrow 0}\left(\left\langle\boldsymbol{v}^{\varepsilon} \cdot \boldsymbol{n}, \psi\right\rangle_{\partial \Omega, T}-\left\langle\boldsymbol{v}^{\varepsilon}, \nabla \psi\right\rangle_{\Omega, T}\right)=\left\langle v_{D}, \psi\right\rangle_{\partial \Omega, T}-\langle\boldsymbol{v}, \nabla \psi\rangle_{\Omega, T},
$$

and, together with $\operatorname{div} \boldsymbol{v}=0$ in $\Omega_{T}$, given by (5.5), conclude $\boldsymbol{v} \cdot \boldsymbol{n}=v_{D}$ on $(0, T) \times \partial \Omega$.

Considering $\psi_{1}, \psi_{2} \in C_{0}^{\infty}\left(\Omega_{T} ; C_{p e r}^{\infty}(Y)\right)$ with $\psi_{1}(t, x, y)=0$ in $Y \backslash Y_{s}$, such that $\psi_{1} \times \boldsymbol{n}=0$ on $\Gamma_{a s}$, and $\psi_{2}(t, x, y)=0$ in $Y \backslash Y_{a}$, for $(t, x) \in \Omega_{T}$, taking $\psi^{\varepsilon}(t, x)=\varepsilon\left(\psi_{1}(t, x, x / \varepsilon), \psi_{2}(t, x, x / \varepsilon), \psi_{1}(t, x, x / \varepsilon)\right)$ as a test function in (4.1) and applying two-scale convergences of $\boldsymbol{v}_{j}^{\varepsilon}$, with $j=z, a, s p$ and of $p_{l}^{\varepsilon}$, with $l=a, s$, stated in Lemma 5.1, yield

$$
\left\langle p_{s}, \operatorname{div}_{y} \psi_{1}\right\rangle_{\Omega \times Y_{s}, T}+\left\langle p_{a}, \operatorname{div}_{y} \psi_{2}\right\rangle_{\Omega \times Y_{a}, T}=0 .
$$

This implies that $p_{l} \in L^{2}\left(\Omega_{T} ; H^{1}\left(Y_{l}\right)\right)$ with $\nabla_{y} p_{l}=0$ a.e. in $\Omega_{T} \times Y_{l}$, where $l=a, s$. Thus $p_{l}$ is independent of $y$ in $\Omega_{T} \times Y_{l}$ and $p_{l}=p_{l}(t, x)$ a.e. in $\Omega_{T} \times Y_{l}$, where $l=a, s$. Taking now $\psi^{\varepsilon}(t, x)=$ $\varepsilon\left(\psi_{1}(t, x, x / \varepsilon), \psi_{1}(t, x, x / \varepsilon), 0\right)$ with $\psi_{1} \in C_{0}^{\infty}\left(\Omega_{T} ; C_{p e r}^{\infty}(Y)\right)$ and $\psi_{1}(t, x, y)=0$ in $\bar{Y}_{a s}$ and $\psi_{1} \times \boldsymbol{n}=0$ on $\Gamma_{z}$ as a test function in (4.1) and letting $\varepsilon \rightarrow 0$ give

$$
\left\langle p_{a}(t, x)-p_{s}(t, x), \psi_{1}(t, x, y) \cdot \boldsymbol{n}\right\rangle_{\Omega \times \Gamma_{z}, T}=0 .
$$

Thus $p_{a}(t, x)=p_{s}(t, x)$ a.e. in $\Omega_{T} \times \Gamma_{z}$ and $p_{a}(t, x)=p_{s}(t, x)=p(t, x)$ a.e. in $\Omega_{T}$. From the relation $\left\langle p_{a}, 1\right\rangle_{\Omega \times Y_{a}}+\left\langle p_{s}, 1\right\rangle_{\Omega \times Y_{s}}=0$ in Lemma 5.1 we obtain that $\langle p(t, x), 1\rangle_{\Omega}=0$ for a.a. $t \in(0, T)$.

Now we consider $\psi^{\varepsilon}(t, x)=\left(\psi_{1}(t, x, x / \varepsilon)+\psi_{2}(t, x, x / \varepsilon), \psi_{1}(t, x, x / \varepsilon), \psi_{2}(t, x, x / \varepsilon)\right)$, where $\psi_{1}, \psi_{2} \in$ $C_{0}^{\infty}\left(\Omega_{T}, C_{p e r}^{\infty}(Y)\right)$ with $\psi_{2} \cdot \boldsymbol{n}=0$ on $\Gamma_{z} \cup \Gamma_{a w}$ and $\psi_{2} \times \boldsymbol{n}=-\psi_{1} \times \boldsymbol{n}$ on $\Gamma_{z s}$ and $\operatorname{div}_{y} \psi_{1}=0$ in $\Omega_{T} \times Y$, $\operatorname{div}_{y} \psi_{2}=0$ in $\Omega_{T} \times Y_{s}$ as a test function in (4.1). The two-scale convergences in (5.1) and (5.2), and the convergence of $c_{a}^{\varepsilon}$ and $c_{s}^{\varepsilon}$ on $(0, T) \times \Gamma_{z s}^{\varepsilon}$ stated in (5.3) imply

$$
\begin{aligned}
&\left\langle 2 \eta \mathrm{S}_{y} \boldsymbol{v}_{z}, \mathrm{~S}_{y}\left(\psi_{1}+\psi_{2}\right)\right\rangle_{Y_{z} \times \Omega_{T}}-\left\langle p, \operatorname{div}_{x}\left(\psi_{1}+\psi_{2}\right)\right\rangle_{Y_{z} \times \Omega_{T}}+\left\langle K_{a}^{-1}(y) \boldsymbol{v}_{a}, \psi_{1}\right\rangle_{Y_{a} \times \Omega_{T}} \\
&-\left\langle p, \operatorname{div}_{x} \psi_{1}\right\rangle_{Y_{a} \times \Omega_{T}}+\left\langle K_{s p}^{-1}(y) \boldsymbol{v}_{s p}, \psi_{2}\right\rangle_{Y_{a s} \times \Omega_{T}}-\left\langle p, \operatorname{div}_{x} \psi_{2}\right\rangle_{Y_{a s} \times \Omega_{T}} \\
&+\left\langle\delta(y)\left(c_{s}-c_{a}\right)+\kappa(y) \boldsymbol{v}_{a} \cdot \boldsymbol{n}, \psi_{1} \cdot \boldsymbol{n}\right\rangle_{\Gamma_{z s} \times \Omega_{T}}=0,
\end{aligned}
$$

where $\mathrm{S}_{y} \boldsymbol{v}=1 / 2\left(\nabla_{y} \boldsymbol{v}+\nabla_{y} \boldsymbol{v}^{T}\right)$. Choosing $\psi_{1}=0$, restricting $\psi_{2}$ to $Y_{z}$, i.e. $\psi_{2} \in C_{0}^{\infty}\left(\Omega_{T}, C_{0}^{\infty}\left(Y_{z}\right)\right)$, and applying the integration by parts imply

$$
\left\langle-2 \eta \operatorname{div}_{y}\left(\mathrm{~S}_{y} \boldsymbol{v}_{z}\right)+\nabla_{x} p, \psi_{2}\right\rangle_{\Omega_{T} \times Y_{z}}=0 .
$$

Since $\operatorname{div}_{y} \psi_{2}(t, x, y)=0$ in $\Omega_{T} \times Y_{z}$, there exists $p_{1, z} \in L^{2}\left(\Omega_{T}, L_{\mathrm{per}}^{2}\left(Y_{z}\right) / \mathbb{R}\right)$, see $[17,19]$, such that

$$
-2 \eta \operatorname{div}_{y}\left(\mathrm{~S}_{y} \boldsymbol{v}_{z}\right)+\nabla_{x} p+\nabla_{y} p_{1, z}=0 \quad \text { in } \Omega_{T} \times Y_{z} .
$$

Similarly we obtain the existence of $p_{1, a} \in L^{2}\left(\Omega_{T}, L_{\mathrm{per}}^{2}\left(Y_{a}\right) / \mathbb{R}\right), p_{1, s p} \in L^{2}\left(\Omega_{T}, L_{\mathrm{per}}^{2}\left(Y_{a s}\right) / \mathbb{R}\right)$ and

$$
\begin{aligned}
K_{a}^{-1} \boldsymbol{v}_{a}+\nabla_{x} p+\nabla_{y} p_{1, a}=0 & \text { in } \Omega_{T} \times Y_{a}, \\
K_{s p}^{-1} \boldsymbol{v}_{s p}+\nabla_{x} p+\nabla_{y} p_{1, s p}=0 & \text { in } \Omega_{T} \times Y_{a s} .
\end{aligned}
$$

Considering $\operatorname{div} \boldsymbol{v}_{j}^{\varepsilon} \in L^{2}\left((0, T) \times \Omega_{j}^{\varepsilon}\right)$ and $\operatorname{div} \boldsymbol{v}_{j}^{\varepsilon}=0$ in $(0, T) \times \Omega_{j}^{\varepsilon}$, with $j=z, a, s p$ and $\Omega_{s p}^{\varepsilon}:=\Omega_{a s}^{\varepsilon}$, we obtain for $\psi \in C_{0}^{\infty}\left((0, T) \times \Omega ; C_{0}^{\infty}\left(Y_{j}\right)\right)$, where $Y_{s p}:=Y_{a s}$,

$$
0=\lim _{\varepsilon \rightarrow 0}\left\langle\operatorname{div} \boldsymbol{v}_{j}^{\varepsilon}, \psi(t, x, x / \varepsilon)\right\rangle_{\Omega_{j}^{\varepsilon}, T}=\lim _{\varepsilon \rightarrow 0}\left\langle\boldsymbol{v}_{j}^{\varepsilon}, \nabla_{x} \psi(t, x, x / \varepsilon)+\varepsilon^{-1} \nabla_{y} \psi(t, x, x / \varepsilon)\right\rangle_{\Omega_{j}^{\varepsilon}, T} .
$$

The two-scale convergence of $\boldsymbol{v}_{j}^{\varepsilon}$ ensures $\lim _{\varepsilon \rightarrow 0}\left\langle\boldsymbol{v}_{j}^{\varepsilon}(t, x), \nabla_{y} \psi(t, x, x / \varepsilon)\right\rangle_{\Omega_{j}^{\varepsilon}, T}=0$ and then implies that $\operatorname{div}_{y} \boldsymbol{v}_{z}=0$ in $\Omega_{T} \times Y_{z}, \operatorname{div}_{y} \boldsymbol{v}_{s p}=0$ in $\Omega_{T} \stackrel{\varepsilon \rightarrow 0}{\times} Y_{a s}$, and $\operatorname{div}_{y} \boldsymbol{v}_{a}=0$ in $\Omega_{T} \times Y_{a}$. Similarly, using $\operatorname{div} \boldsymbol{v}^{\varepsilon} \in$ $L^{2}\left(\Omega_{T}\right)$ and $\operatorname{div} \boldsymbol{v}^{\varepsilon}=0$ in $\Omega_{T}$, the two-scale convergence of $\boldsymbol{v}^{\varepsilon}$ ensures $\operatorname{div}_{y} \overline{\boldsymbol{v}}=0$ in $\Omega_{T} \times Y$. 
Considering the two-scale convergence of $\boldsymbol{v}_{z}^{\varepsilon}, \boldsymbol{v}_{a}^{\varepsilon}, \boldsymbol{v}_{s p}^{\varepsilon}$, and using the calculations similar to (5.5)-(5.6) we can conclude $\boldsymbol{v}_{z} \cdot \boldsymbol{n}=\left(\boldsymbol{v}_{a}+\boldsymbol{v}_{s p}\right) \cdot \boldsymbol{n}$ on $\Omega_{T} \times \Gamma_{a s}$ and $\boldsymbol{v}_{z} \cdot \boldsymbol{n}=\boldsymbol{v}_{a} \cdot \boldsymbol{n}$ on $\Omega_{T} \times \Gamma_{z}$.

Inequality (4.30), applied to $\boldsymbol{v}_{z}^{\varepsilon}$, with $\Gamma_{z s}^{\varepsilon}$ and $\Omega_{z}^{\varepsilon}$, and the estimates for $\left\|\boldsymbol{v}_{z}^{\varepsilon}\right\|_{L^{2}\left((0, T) \times \Omega_{z}^{\varepsilon}\right)}$ and $\varepsilon\left\|\nabla \boldsymbol{v}_{z}^{\varepsilon}\right\|_{L^{2}\left((0, T) \times \Omega_{z}^{\varepsilon}\right)}$ in (4.12) ensure the uniform in $\varepsilon$ boundedness of $\varepsilon^{1 / 2}\left\|\boldsymbol{v}_{z}^{\varepsilon}\right\|_{L^{2}\left((0, T) \times \Gamma_{z s}^{\varepsilon}\right)}$. Then the compactness theorem for the two-scale convergence on oscillating boundaries, see [28] or Theorem 5.9 in Appendix, together with the two-scale convergence of $\varepsilon \nabla \boldsymbol{v}_{z}^{\varepsilon}$ and $\operatorname{div} \boldsymbol{v}_{z}^{\varepsilon}=0$, as well as $\operatorname{div}_{y} \boldsymbol{v}_{z}=0$, implies the two-scale convergence of $\boldsymbol{v}_{z}^{\varepsilon}$ to $\boldsymbol{v}_{z}$ on $\Omega_{T} \times \Gamma_{z s}$ and ensures $\boldsymbol{v}_{z} \times \boldsymbol{n}=0$ on $\Omega_{T} \times \Gamma_{z s}$.

Applying in (5.7) integration by parts and accounting equations (5.8) and (5.9) yield

$$
\begin{aligned}
\left\langle 2 \eta\left(\mathrm{S}_{y} \boldsymbol{v}_{z} \boldsymbol{n}\right) \cdot \boldsymbol{n}-p_{1, z}, \psi_{1} \cdot \boldsymbol{n}\right\rangle_{\Omega_{T} \times \Gamma_{z}}+\left\langle 2 \eta\left(\mathrm{S}_{y} \boldsymbol{v}_{z} \boldsymbol{n}\right) \cdot \boldsymbol{n}-p_{1, z},\left(\psi_{1}+\psi_{2}\right) \cdot \boldsymbol{n}\right\rangle_{\Omega_{T} \times \Gamma_{a s}} & \\
+ & \left\langle p_{1, a}+\delta(y)\left(c_{s}-c_{a}\right)+\kappa(y) \boldsymbol{v}_{a} \cdot \boldsymbol{n}, \psi_{1} \cdot \boldsymbol{n}\right\rangle_{\Omega_{T} \times \Gamma_{z s}}+\left\langle p_{1, s p}, \psi_{2} \cdot \boldsymbol{n}\right\rangle_{\Omega_{T} \times \Gamma_{a s}}=0
\end{aligned}
$$

for $\psi_{1}, \psi_{2} \in C_{0}^{\infty}\left(\Omega_{T}, C_{p e r}^{\infty}(Y)\right)$ with $\psi_{2} \cdot \boldsymbol{n}=0$ on $\Gamma_{z} \cup \Gamma_{a w}$ and $\psi_{2} \times \boldsymbol{n}=-\psi_{1} \times \boldsymbol{n}$ on $\Gamma_{z s}, \operatorname{div}_{y} \psi_{1}=0$ in $\Omega_{T} \times Y, \operatorname{div}_{y} \psi_{2}=0$ in $\Omega_{T} \times Y_{s}$. Thus, we conclude that $\boldsymbol{v}_{z} \in L^{2}\left(\Omega_{T} ; H_{\mathrm{per}}^{1}\left(Y_{z}\right) / \mathbb{R}\right), \boldsymbol{v}_{s p} \in L^{2}\left(\Omega_{T} \times Y_{a s}\right)$, $\boldsymbol{v}_{a} \in L^{2}\left(\Omega_{T} \times Y_{a}\right)$, and $p \in L^{2}\left(0, T ; L_{0}^{2}(\Omega)\right), p_{1, z} \in L^{2}\left(\Omega_{T} ; L_{\mathrm{per}}^{2}\left(Y_{z}\right) / \mathbb{R}\right), p_{1, s p} \in L^{2}\left(\Omega_{T} ; L_{\mathrm{per}}^{2}\left(Y_{a s}\right) / \mathbb{R}\right)$, and $p_{1, a} \in L^{2}\left(\Omega_{T} ; L_{\mathrm{per}}^{2}\left(Y_{a}\right) / \mathbb{R}\right)$, satisfy equations

$$
\left\{\begin{array}{cl}
-\eta \Delta_{y} \boldsymbol{v}_{z}+\nabla_{x} p+\nabla_{y} p_{1, z}=0 & \text { in } \Omega_{T} \times Y_{z}, \\
K_{a}^{-1} \boldsymbol{v}_{a}+\nabla_{x} p+\nabla_{y} p_{1, a}=0 & \text { in } \Omega_{T} \times Y_{a}, \\
K_{s p}^{-1} \boldsymbol{v}_{s p}+\nabla_{x} p+\nabla_{y} p_{1, s p}=0 & \text { in } \Omega_{T} \times Y_{a s}, \\
\operatorname{div}_{y} \boldsymbol{v}_{z}=0 \text { in } \Omega_{T} \times Y_{z}, \operatorname{div}_{y} \boldsymbol{v}_{a}=0 \text { in } \Omega_{T} \times Y_{a}, \quad \operatorname{div}_{y} \boldsymbol{v}_{s p}=0 & \text { in } \Omega_{T} \times Y_{a s},
\end{array}\right.
$$

with boundary and transmission conditions

$$
\begin{cases}-2 \eta\left(\mathrm{S}_{y} \boldsymbol{v}_{z} \boldsymbol{n}\right) \cdot \boldsymbol{n}+p_{1, z}-p_{1, a}=\delta(y)\left(c_{s}-c_{a}\right)+\kappa(y) \boldsymbol{v}_{a} \cdot \boldsymbol{n} & \text { on } \Omega_{T} \times \Gamma_{z s} \\ -2 \eta\left(\mathrm{S}_{y} \boldsymbol{v}_{z} \boldsymbol{n}\right) \cdot \boldsymbol{n}+p_{1, z}=p_{1, s p} & \text { on } \Omega_{T} \times \Gamma_{a s} \\ \boldsymbol{v}_{z} \cdot \boldsymbol{n}=\boldsymbol{v}_{a} \cdot \boldsymbol{n}+\boldsymbol{v}_{s p} \cdot \boldsymbol{n}, \boldsymbol{v}_{z} \times \boldsymbol{n}=0 & \text { on } \Omega_{T} \times \Gamma_{a s} \\ \boldsymbol{v}_{z} \cdot \boldsymbol{n}=\boldsymbol{v}_{a} \cdot \boldsymbol{n}, \quad \boldsymbol{v}_{z} \times \boldsymbol{n}=0 & \text { on } \Omega_{T} \times \Gamma_{z} \\ \boldsymbol{v}_{s p} \cdot \boldsymbol{n}_{a w}=0 & \text { on } \Omega_{T} \times \Gamma_{a w}\end{cases}
$$

Considering the structure, linearity, and uniqueness of a solution of equations (5.10) and (5.11), the proof of which follows the same lines as for microscopic model (4.1), we can express $\boldsymbol{v}_{l}$ and $p_{1, l}$, with $l=z, a$ or $s p$, in the form

$$
\left\{\begin{array}{lll}
\boldsymbol{v}_{z}=-\sum_{i=1}^{3} \partial_{x_{i}} p \boldsymbol{w}_{z}^{i}+\left(c_{s}-c_{a}\right) \boldsymbol{r}_{z}, & \boldsymbol{v}_{a}=-\sum_{i=1}^{3} \partial_{x_{i}} p \boldsymbol{w}_{a}^{i}+\left(c_{s}-c_{a}\right) \boldsymbol{r}_{a}, & \boldsymbol{v}_{s p}=-\sum_{i=1}^{3} \partial_{x_{i}} p \boldsymbol{w}_{s p}^{i}, \\
p_{1, z}=-\sum_{i=1}^{3} \partial_{x_{i}} p \pi_{z}^{i}+\left(c_{s}-c_{a}\right) \zeta_{z}, & p_{1, a}=-\sum_{i=1}^{3} \partial_{x_{i}} p \pi_{a}^{i}+\left(c_{s}-c_{a}\right) \zeta_{a}, & p_{1, s p}=-\sum_{i=1}^{3} \partial_{x_{i}} p \pi_{s p}^{i},
\end{array}\right.
$$

where $\boldsymbol{w}_{l}^{i}, \pi_{l}^{i}$, with $l=z, a$ and $s p$, are solutions of the unit cell problems

$$
\left\{\begin{aligned}
-\eta \Delta_{y} \boldsymbol{w}_{z}^{i}+\nabla_{y} \pi_{z}^{i}=e_{i} & \text { in } Y_{z}, \\
K_{a}^{-1} \boldsymbol{w}_{a}^{i}+\nabla_{y} \pi_{a}^{i}=e_{i} & \text { in } Y_{a}, \\
K_{s p}^{-1} \boldsymbol{w}_{s p}^{i}+\nabla_{y} \pi_{s p}^{i}=e_{i} & \text { in } Y_{a s}, \\
\operatorname{div}_{y} \boldsymbol{w}_{z}^{i}=0 \quad \text { in } Y_{z}, & \operatorname{div}_{y} \boldsymbol{w}_{a}^{i}=0 \quad \text { in } Y_{a}, \quad \operatorname{div}_{y} \boldsymbol{w}_{s p}^{i}=0 \quad \text { in } Y_{a s},
\end{aligned}\right.
$$

with transmission conditions

$$
\begin{cases}-2 \eta\left(\mathrm{S}_{y} \boldsymbol{w}_{z}^{i} \boldsymbol{n}\right) \cdot \boldsymbol{n}+\pi_{z}^{i}-\pi_{a}^{i}=\kappa(y) \boldsymbol{w}_{a}^{i} \cdot \boldsymbol{n} & \text { on } \Gamma_{z s} \\ -2 \eta\left(\mathrm{S}_{y} \boldsymbol{w}_{z}^{i} \boldsymbol{n}\right) \cdot \boldsymbol{n}+\pi_{z}^{i}=\pi_{s p}^{i} & \text { on } \Gamma_{a s} \\ \boldsymbol{w}_{z}^{i} \cdot \boldsymbol{n}=\left(\boldsymbol{w}_{a}^{i}+\boldsymbol{w}_{s p}^{i}\right) \cdot \boldsymbol{n}, \quad \boldsymbol{w}_{z}^{i} \times \boldsymbol{n}=0 & \text { on } \Gamma_{a s} \\ \boldsymbol{w}_{z}^{i} \cdot \boldsymbol{n}=\boldsymbol{w}_{a}^{i} \cdot \boldsymbol{n}, & \text { on } \Gamma_{z} \\ \boldsymbol{w}_{s p}^{i} \cdot \boldsymbol{n}_{a w}=0 & \text { on } \Gamma_{a w}\end{cases}
$$


and $\boldsymbol{r}_{l}, \zeta_{l}$, with $l=z$ and $a$, solve the following problem

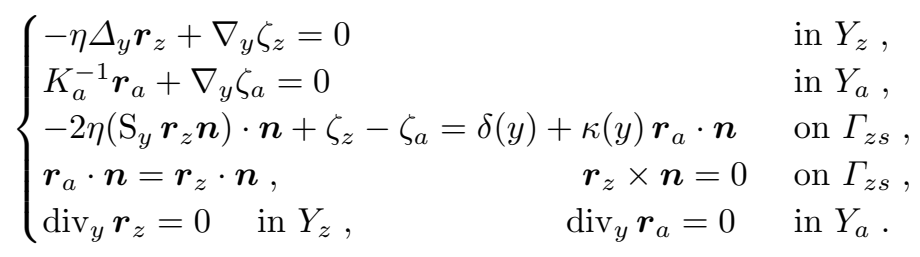

Averaging the expressions in (5.12) over $Y_{z}, Y_{a}$ and $Y_{a s}$, respectively, using the definition of $\boldsymbol{v}$ and defining the vector $\boldsymbol{M}$ and the permeability tensor $\boldsymbol{K}=\left(K_{i j}\right)_{1 \leq i, j \leq 3}$ by

$$
\boldsymbol{M}=\frac{1}{|Y|}\left(\int_{Y_{z}} \boldsymbol{r}_{z} d y+\int_{Y_{a}} \boldsymbol{r}_{a} d y\right), \quad K_{i j}=\frac{1}{|Y|}\left(\int_{Y_{z}} \boldsymbol{w}_{z, j}^{i} d y+\int_{Y_{a}} \boldsymbol{w}_{a, j}^{i} d y+\int_{Y_{a s}} \boldsymbol{w}_{s p, j}^{i} d y\right),
$$

we obtain the equation for $\boldsymbol{v}$ stated in (5.4).

For concentrations of a osmotically active solute and transporters on the cell membrane we obtain the following macroscopic problem

Theorem 5.4. The sequence of solutions of the microscopic model (3.2)-(3.7), (3.9) converges to a unique solution $c_{l} \in H^{1}\left(0, T ; H^{1}(\Omega)\right) \cap L^{\infty}\left(\Omega_{T}\right), \vartheta_{j, l} \in H^{1}\left(0, T ; L^{2}\left(\Omega \times \Gamma_{z s}\right)\right) \cap L^{\infty}\left(\Omega_{T} \times \Gamma_{z s}\right)$, with $l=a, s$, $j=f, b$, of the initial boundary values problem in $\Omega_{T}=(0, T) \times \Omega$

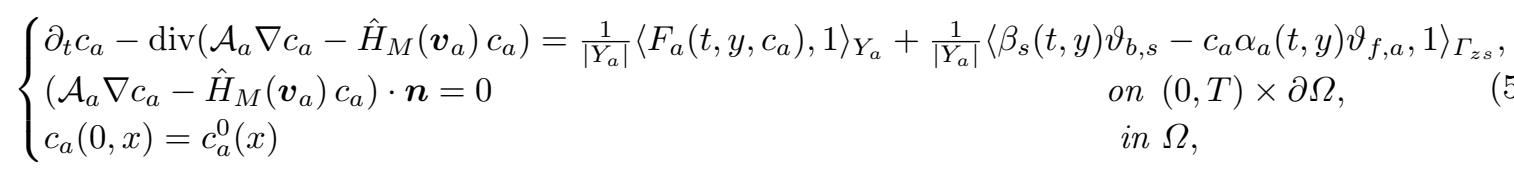

and

$$
\begin{cases}\partial_{t} c_{s}-\operatorname{div}\left(\mathcal{A}_{s} \nabla c_{s}-\hat{H}_{M}\left(\boldsymbol{v}_{s}\right) c_{s}\right)=\frac{1}{\left|Y_{s}\right|}\left\langle F_{s}\left(t, y, c_{s}\right), 1\right\rangle_{Y_{s}}+\frac{1}{\left|Y_{s}\right|}\left\langle\beta_{a}(t, y) \vartheta_{b, a}-c_{s} \alpha_{s}(t, y) \vartheta_{f, s}, 1\right\rangle_{\Gamma_{z s}} \\ \left(\mathcal{A}_{s} \nabla c_{s}-\hat{H}_{M}\left(\boldsymbol{v}_{s}\right) c_{s}\right) \cdot \boldsymbol{n}=0 & \text { on }(0, T) \times \partial \Omega \\ c_{s}(0, x)=c_{s}^{0}(x) & \text { in } \Omega\end{cases}
$$

where homogenized diffusion coefficients $\mathcal{A}_{a}, \mathcal{A}_{s}$ and velocity fields $\hat{H}_{M}\left(\boldsymbol{v}_{a}\right), \hat{H}_{M}\left(\boldsymbol{v}_{s}\right)$ are defined by (5.20) with $\boldsymbol{v}_{a}$ and $\boldsymbol{v}_{s}$ given as solutions of (5.10), (5.11), and transporter concentrations satisfy ordinary differential equations, for $l=a, s$,

$$
\begin{cases}\partial_{t} \vartheta_{f, l}=R_{l}\left(t, y, \vartheta_{f, l}\right)-\alpha_{l}(t, y) c_{l} \vartheta_{f, l}+\beta_{l}(t, y) \vartheta_{b, l}-\gamma_{f, l}(t, y) \vartheta_{f, l} & \text { on } \Omega_{T} \times \Gamma_{z s} \\ \partial_{t} \vartheta_{b, l}=\quad \alpha_{l}(t, y) c_{l} \vartheta_{f, l}-\beta_{l}(t, y) \vartheta_{b, l}-\gamma_{b, l}(t, y) \vartheta_{b, l} & \text { on } \Omega_{T} \times \Gamma_{z s} \\ \vartheta_{f, l}(0, x, y)=\vartheta_{f, l}^{0}(x, y), \vartheta_{b, l}(0, x, y)=\vartheta_{b, l}^{0}(x, y) & \text { on } \Omega \times \Gamma_{z s}\end{cases}
$$

Proof. To derive macroscopic equations we shall apply two-scale and strong convergences stated in Lemmata 5.1 and 5.2. The Lipschitz continuity of $F_{l}$ and the strong $L^{2}$-convergence of $c_{l}^{\varepsilon}$ imply $F_{l}^{\varepsilon}\left(t, x, c_{l}^{\varepsilon}\right) \rightarrow F_{l}\left(t, y, c_{l}\right)$ two-scale in $\Omega_{T} \times Y_{l}$, with $l=a, s$. Taking $\varphi(t, x)=\psi_{1}(t, x)+\varepsilon \psi_{2}(t, x, x / \varepsilon)$ with $\psi_{1} \in L^{2}\left(0, T ; H^{1}(\Omega)\right)$ and $\psi_{2} \in C_{0}^{\infty}\left(\Omega_{T} ; C_{p e r}^{\infty}(Y)\right)$ as a test function in (4.2) and (4.3), applying two-scale convergences and strong convergence in $L^{2}\left(\Omega_{T}\right)$ of $c_{l}^{\varepsilon}$, together with two-scale convergences for $\boldsymbol{v}_{l}^{\varepsilon}$ and $\vartheta_{j, l}^{\varepsilon}$, where $j=f, b$ and $l=a, s$, and the linearity of $H_{M}$, considering appropriate subsequences and passing to the limit as $\varepsilon \rightarrow 0$, imply

$$
\begin{array}{r}
\left|Y_{l}\right|\left\langle\partial_{t} c_{l}, \psi_{1}\right\rangle_{\Omega_{T}}+\left\langle D_{l}(t, y)\left(\nabla c_{l}+\nabla_{y} c_{l}^{1}\right)-H_{M}\left(\boldsymbol{v}_{l}(t, x, y)\right) c_{l}, \nabla \psi_{1}+\nabla_{y} \psi_{2}\right\rangle_{\Omega_{T} \times Y_{l}} \\
=\left\langle\beta_{l-1}(t, y) \vartheta_{b, l-1}-\alpha_{l}(t, y) c_{l} \vartheta_{f, l}, \psi_{1}\right\rangle_{\Omega_{T} \times \Gamma_{z s}}+\left\langle F_{l}\left(t, y, c_{l}\right), \psi_{1}\right\rangle_{\Omega_{T} \times Y_{l}}
\end{array}
$$


where $l=a, s$, with $a-1:=s$ and $s-1:=a$. Choosing now $\psi_{1}=0$ in (5.17) we obtain

$$
\left\langle D_{l}(t, y)\left(\nabla c_{l}+\nabla_{y} c_{l}^{1}\right)-H_{M}\left(\boldsymbol{v}_{l}\right) c_{l}, \nabla_{y} \psi_{2}\right\rangle_{\Omega_{T} \times Y_{l}}=0, \quad \text { with } l=a, s,
$$

for all $\psi_{2} \in C_{0}^{\infty}\left(\Omega_{T} ; C_{\mathrm{per}}^{\infty}(Y)\right)$ and can conclude that $c_{l}^{1}$ depends linearly on $\nabla c_{l}$ and $c_{l}$. Thus we can consider an Ansatz

$$
c_{l}^{1}(t, x, y)=\sum_{i=1}^{3} \frac{\partial c_{l}(t, x)}{\partial x_{i}} \omega_{l}^{i}(y)+c_{l}(t, x) z_{l}(t, x, y),
$$

where $l=a, s$, and $\omega_{l}^{i}$ and $z_{l}$ are solutions of the cell problems

$$
\left\{\begin{array}{cl}
-\operatorname{div}_{y}\left(D_{l}(t, y) \nabla_{y} \omega_{l}^{i}\right)=\sum_{k=1}^{3} \partial_{y_{k}} D_{l, k i}(t, y) & \text { in }(0, T) \times Y_{l}, \\
-D_{l}(t, y) \nabla_{y} \omega_{l}^{i} \cdot \boldsymbol{n}=\sum_{k=1}^{3} D_{l, k i}(t, y) \boldsymbol{n}_{k} & \text { in }(0, T) \times\left(\partial Y_{l} \backslash \partial Y\right), \\
\omega_{l}^{i} \text { and }\left.D_{l}(t, y) \nabla_{y} \omega_{l}^{i} \cdot \boldsymbol{n}\right|_{\partial Y_{l} \cap \partial Y} & \text { are } Y-\text { periodic }
\end{array}\right.
$$

and

$$
\left\{\begin{aligned}
\operatorname{div}_{y}\left(D_{l}(t, y) \nabla_{y} z_{l}-H_{M}\left(\boldsymbol{v}_{l}\right)\right)=0 & \text { in } \Omega_{T} \times Y_{l}, \\
\left(D_{l}(t, y) \nabla_{y} z_{l}-H_{M}\left(\boldsymbol{v}_{l}\right)\right) \cdot \boldsymbol{n}=0 & \text { on } \Omega_{T} \times\left(\partial Y_{l} \backslash \partial Y\right), \\
z_{l} \text { and }\left.D_{l}(t, y) \nabla_{y} z_{l} \cdot \boldsymbol{n}\right|_{\partial Y_{l} \cap \partial Y} & \text { are } Y \text { - periodic }
\end{aligned}\right.
$$

Next, setting $\psi_{2}=0$ in (5.17) yields macroscopic equations (5.14)-(5.15) for $c_{s}$ and $c_{a}$, where homogenized diffusion matrices and macroscopic velocity fields are given by

$$
\begin{aligned}
\mathcal{A}_{l, i j}(t) & =\frac{1}{\left|Y_{l}\right|} \int_{Y_{l}}\left(D_{l, i j}(t, y)+\sum_{k=1}^{3} D_{l, i k}(t, y) \partial_{y_{k}} \omega_{l}^{j}(y)\right) d y \quad \text { for } l=a, s, \\
\hat{H}_{M}\left(\boldsymbol{v}_{l}\right)_{i}(t, x) & =\frac{1}{\left|Y_{l}\right|} \int_{Y_{l}} H_{M}\left(\boldsymbol{v}_{l}(t, x, y)\right)_{i} d y-\frac{1}{\left|Y_{l}\right|} \int_{Y_{l}} \sum_{k=1}^{3} D_{l, i k}(t, y) \partial_{y_{k}} z_{l}(t, x, y) d y,
\end{aligned}
$$

whereas $w_{l}^{j}$ and $z_{l}$ are solutions of the unit cell problems (5.18) and (5.19).

Applying the boundary unfolding operator $\mathcal{T}_{\Gamma}^{\varepsilon}$ to (3.5) and testing with $\psi \in L^{2}\left(\Omega_{T} \times \Gamma_{z s}\right)$ give

$$
\begin{array}{r}
\left\langle\partial_{t} \mathcal{T}_{\Gamma}^{\varepsilon}\left(\vartheta_{f, l}^{\varepsilon}\right), \psi\right\rangle_{\Omega \times \Gamma_{z s}, T}=\left\langle R_{l}\left(t, y, \mathcal{T}_{\Gamma}^{\varepsilon}\left(\vartheta_{f, l}^{\varepsilon}\right)\right), \psi\right\rangle_{\Omega \times \Gamma_{z s}, T}-\left\langle\alpha_{l}(t, y) \mathcal{T}_{\Gamma}^{\varepsilon}\left(\vartheta_{f, l}^{\varepsilon}\right) \mathcal{T}_{\Gamma}^{\varepsilon}\left(c_{l}^{\varepsilon}\right), \psi\right\rangle_{\Omega \times \Gamma_{z s}, T} \\
+\left\langle\beta_{l}(t, y) \mathcal{T}_{\Gamma}^{\varepsilon}\left(\vartheta_{b, l}^{\varepsilon}\right)-\gamma_{f, l}(t, y) \mathcal{T}_{\Gamma}^{\varepsilon}\left(\vartheta_{f, l}^{\varepsilon}\right), \psi\right\rangle_{\Omega \times \Gamma_{z s}, T} \\
\left\langle\partial_{t} \mathcal{T}_{\Gamma}^{\varepsilon}\left(\vartheta_{b, l}^{\varepsilon}\right), \psi\right\rangle_{\Omega \times \Gamma_{z s}, T}=\left\langle\alpha_{l}(t, y) \mathcal{T}_{\Gamma}^{\varepsilon}\left(\vartheta_{f, l}^{\varepsilon}\right) \mathcal{T}_{\Gamma}^{\varepsilon}\left(c_{l}^{\varepsilon}\right)-\left(\beta_{l}(t, y)+\gamma_{b, l}(t, y)\right) \mathcal{T}_{\Gamma}^{\varepsilon}\left(\vartheta_{b, l}^{\varepsilon}\right), \psi\right\rangle_{\Omega \times \Gamma_{z s}, T}
\end{array}
$$

Considering the strong convergence of $\mathcal{T}_{\Gamma}^{\varepsilon}\left(\vartheta_{f, l}^{\varepsilon}\right)$ and $\mathcal{T}_{\Gamma}^{\varepsilon}\left(c_{l}^{\varepsilon}\right)$ stated in (5.3), the equivalence between two-scale convergence and the weak convergence of the unfolded sequence, and Lipschitz continuity of $R_{l}$, with $l=a, s$, we can pass in (5.21) to the limit as $\varepsilon \rightarrow 0$ and obtain equations (5.16). The assumption on the initial data, the similar arguments as in the proof of Lemma 5.2, the two-scale convergence of $c_{l}^{\varepsilon}, \vartheta_{j, l}^{\varepsilon}$ and of their time derivatives ensure that the initial conditions for $c_{l}$ and $\vartheta_{j, l}$, with $l=a, s$ and $j=f, b$, are satisfied in $L^{2}(\Omega)$ and in $L^{2}\left(\Omega \times \Gamma_{z s}\right)$, respectively. The proof of the uniqueness follows along the same lines as for the microscopic model and implies the convergence of the entire sequence of solutions of the microscopic problems.

\section{Appendix}

We recall here the definition of the two-scale convergence and unfolding operator. 
Definition 5.5. $[2,29]$ A sequence $\left\{u^{\varepsilon}\right\} \subset L^{2}((0, T) \times \Omega)$ is said to two-scale converge to a limit $u_{0} \in$ $L^{2}((0, T) \times \Omega \times Y)$ iff for any $\phi \in L^{2}\left((0, T) \times \Omega, C_{p e r}(Y)\right)$ we have

$$
\lim _{\varepsilon \rightarrow 0} \int_{0}^{T} \int_{\Omega} u^{\varepsilon}(t, x) \phi(t, x, x / \varepsilon) d x d t=\frac{1}{|Y|} \int_{0}^{T} \int_{\Omega} \int_{Y} u_{0}(t, x, y) \phi(t, x, y) d y d x d t .
$$

Theorem 5.6. [2, 29] From each bounded sequence $\left\{u^{\varepsilon}\right\}$ in $L^{2}((0, T) \times \Omega)$ we can extract a subsequence, which two-scale converges to $u_{0} \in L^{2}((0, T) \times \Omega \times Y)$

Theorem 5.7. [2,29] 1. Let $\left\{u^{\varepsilon}\right\}$ be a bounded sequence in $L^{2}\left(0, T ; H^{1}(\Omega)\right)$, which converges weakly to $u \in L^{2}\left(0, T ; H^{1}(\Omega)\right)$. Then, there exists $u_{1} \in L^{2}\left(\Omega_{T} ; H_{\text {per }}^{1}(Y) / \mathbb{R}\right)$ such that, up to a subsequence, $u^{\varepsilon}$ two-scale converges to $u$ and $\nabla u^{\varepsilon}$ two-scale converges to $\nabla u+\nabla_{y} u_{1}$.

2. Let $\left\{u^{\varepsilon}\right\}$ and $\left\{\varepsilon \nabla u^{\varepsilon}\right\}$ be bounded sequences in $L^{2}\left(\Omega_{T}\right)$. Then, there exists $u_{0} \in L^{2}\left(\Omega_{T}, H_{\text {per }}^{1}(Y) / \mathbb{R}\right)$ such that, up to a subsequence, $u^{\varepsilon}$ and $\varepsilon \nabla u^{\varepsilon}$ two-scale converge to $u_{0}$ and $\nabla_{y} u_{0}$, respectively.

Definition 5.8. [28] A sequence $\left\{w^{\varepsilon}\right\} \subset L^{2}\left((0, T) \times \Gamma_{z s}^{\varepsilon}\right)$ is said to two-scale converge to a limit $w \in$ $L^{2}\left((0, T) \times \Omega \times \Gamma_{z s}\right)$ iff for every $\psi \in L^{2}\left((0, T) \times \Omega ; C_{p e r}\left(\Gamma_{z s}\right)\right)$ we have

$$
\lim _{\varepsilon \rightarrow 0} \varepsilon \int_{0}^{T} \int_{\Gamma_{z s}^{\varepsilon}} w^{\varepsilon}(t, x) \psi(t, x, x / \varepsilon) d \gamma_{x} d t=\frac{1}{|Y|} \int_{0}^{T} \int_{\Omega} \int_{\Gamma_{z s}} w(t, x, y) \psi(t, x, y) d \gamma_{y} d x d t .
$$

Theorem 5.9. 1. [28] For each sequence $\left\{w^{\varepsilon}\right\} \subset L^{2}\left((0, T) \times \Gamma_{z s}^{\varepsilon}\right)$ with $\varepsilon^{\frac{1}{2}}\left\|w^{\varepsilon}\right\|_{L^{2}\left((0, T) \times \Gamma_{z s}^{\varepsilon}\right)}$ bounded uniformly in $\varepsilon$, there exists a subsequence and $w \in L^{2}\left((0, T) \times \Omega \times \Gamma_{z s}\right)$ such that the subsequence two-scale converges to $w$.

2. [27] If $\left\{w^{\varepsilon}\right\}$ is bounded in $L^{\infty}\left((0, T) \times \Gamma_{z s}^{\varepsilon}\right)$, then the limit $w \in L^{\infty}\left((0, T) \times \Omega \times \Gamma_{z s}\right)$.

Definition 5.10. [10] 1 . For any function $\phi$ Lebesgue-measurable on the perforated domain $\Omega_{l}^{\varepsilon}$, the unfolding operator $\mathcal{T}_{Y_{l}}^{\varepsilon}: \Omega_{l}^{\varepsilon} \rightarrow \Omega \times Y_{l}, l=a, s$, is defined by

$$
\mathcal{T}_{Y_{l}}^{\varepsilon}(\phi)(x, y)= \begin{cases}\phi\left(\varepsilon\left[\frac{x}{\varepsilon}\right]_{Y}+\varepsilon y\right) & \text { a.e. for } y \in Y_{l}, x \in \tilde{\Omega}_{i n t}^{\varepsilon}, \\ 0 & \text { a.e. for } y \in Y_{l}, x \in \Omega \backslash \tilde{\Omega}_{i n t}^{\varepsilon},\end{cases}
$$

where $k:=\left[\frac{x}{\varepsilon}\right]$ denotes the unique integer combination, such that $x-\left[\frac{x}{\varepsilon}\right]$ belongs to $Y_{l}$, and $\tilde{\Omega}_{\text {int }}^{\varepsilon}=$ $\operatorname{Int}\left(\cup_{k \in \mathbb{Z}^{3}}\left\{\varepsilon \overline{Y^{k}}, \varepsilon Y^{k} \subset \Omega\right\}\right)$. We note that for $w \in H^{1}(\Omega)$ it holds that $\mathcal{T}_{Y_{l}}^{\varepsilon}\left(\left.w\right|_{\Omega_{l}^{\varepsilon}}\right)=\left.\mathcal{T}_{Y}^{\varepsilon}(w)\right|_{\Omega \times Y_{l}}$.

2. For any function $\phi$ Lebesgue-measurable on oscillating boundary $\Gamma_{l}^{\varepsilon}, l=z s, a, s$, the boundary unfolding operator $\mathcal{T}_{\Gamma_{l}}^{\varepsilon}: \Gamma_{l}^{\varepsilon} \rightarrow \Omega \times \Gamma_{l}$, is defined by

$$
\mathcal{T}_{\Gamma_{l}}^{\varepsilon}(\phi)(x, y)= \begin{cases}\phi\left(\varepsilon\left[\frac{x}{\varepsilon}\right]_{Y}+\varepsilon y\right) & \text { a.e. for } y \in \Gamma_{l}, x \in \tilde{\Omega}_{i n t}^{\varepsilon}, \\ 0 & \text { a.e. for } y \in \Gamma_{l}, x \in \Omega \backslash \tilde{\Omega}_{i n t}^{\varepsilon} .\end{cases}
$$

Lemma 5.11. [27] If $\left\{\psi^{\varepsilon}\right\} \subset L^{2}\left((0, T) \times \Gamma_{z s}^{\varepsilon}\right)$ converges two-scale to $\psi \in L^{2}\left((0, T) \times \Omega \times \Gamma_{z s}\right)$ and $\left\{\mathcal{T}_{\Gamma_{z s}}^{\varepsilon}\left(\psi^{\varepsilon}\right)\right\} \subset L^{2}\left((0, T) \times \Omega \times \Gamma_{z s}\right)$ converges weakly to $\psi^{*}$ in $L^{2}\left((0, T) \times \Omega \times \Gamma_{z s}\right)$, then $\psi=\psi^{*}$ a.e. in $(0, T) \times \Omega \times \Gamma_{z s}$.

Acknowledgements. The first author was funded by the German Research Foundation [grant number CH 958/1-1].

\section{References}

[1] E. Acerbi, V. Chiado Piat, G. Dal Maso, D. Percivale. An extension theorem from connected sets, and homogenization in general periodic domains. Nonlin. Anal. Theory, Methods, Applic., 18 (1992), 481-496.

[2] G. Allaire. Homogenization and two-scale convergence. SIAM J. Math. Anal., 23 (1992), 1482-1518.

[3] G. Allaire. Homogenization of the Stokes flow in a connected porous medium. Asymptotic Anal., 2 (1989), 203-222. 
[4] T. Arbogast, H. Lehr. Homogenization of a Darcy-Stokes system modeling vuggy porous media. Computat. Geosci., 10 (2006), 291-302.

[5] V. Calvez, J.G. Houot, N. Meunier, A. Raoult, G. Rusnakova. Mathematical and numerical modeling of early atherosclerotic lesions. ESAIM Proc., (2010), 1-18.

[6] A. Chavarría-Krauser, W. Jäger. Barodiffusion effects in bifurcating capillaries. Comput. Visual. Sci., 13 (2010), 121128.

[7] A. Chavarría-Krauser, M. Ptashnyk. Homogenization of long-range auxin transport in plant tissues. Nonlinear Anal. Real World Applic., 11 (2010), 4524-4532.

[8] D. Cioranescu, J. Saint Jean Paulin. Homogenization of reticulated structures. Springer, New York, 1999.

[9] D. Cioranescu, P. Donato. An introduction to Homogenization. Oxfor University Press, New York, 1999.

[10] D. Cioranescu, P. Donato, R. Zaki. The periodic unfolding method in perforated domains. Port. Math., 63 (2006), 467-496.

[11] J. Claus, A. Chavarría-Krauser. Modeling Regulation of Zinc Uptake via ZIP Transporters in Yeast and Plant Roots. PLoS ONE, 7 (2012), e37193.

[12] J. Claus, A. Bohmann, A.Chavarría-Krauser. Zinc Uptake and Radial Transport in Roots of Arabidopsis thaliana: A Modelling Approach to Understand Accumulation. Ann. Bot.-London, doi: 10.1093/aob/mcs263.

[13] J.Claus, A.Chavarría-Krauser. Implications of a zinc uptake and transport model. Plant Sig. Behav., 8 (2013), e24167.

[14] K. Esau. Anatomy of seed plant. Wiley, 1977.

[15] J. Galvis, M. Sarkis. Non-matching mortar discretization analysis for the coupling Stokes-Darcy equations. Elect. Trans. Numer. Ana., 26 (2007), 350-384.

[16] V. Giovangigli. Multicomponent flow modeling. Birkhäuser, 1999.

[17] V. Girault, P.-A. Raviart. Finite element methods for Navier-Stokes equations: Theory and algorithms. Springer, Berlin Heidelberg, 1986.

[18] T.H. van den Honert. Water transport in plants as a catenary process. Discuss. Faraday Soc., 3 (1948), 146 - 153.

[19] U. Hornung. Homogenization and porous media. Springer-Verlag, 1997.

[20] H. Javot, C. Maurel. The role of aquaporins in root water uptake. Ann. Bot.-London, 90 (2002), 301-313.

[21] W. Jäger, A. Mikelic. On the interface boundary condition of Beavers, Joseph and Saffman. SIAM J. Appl.Math., 60 (2000), 1111-1127.

[22] O.A. Ladyzenskaja, V.A Solonnikov, N.N. Uralceva. Linear and Quasi-linear Equations of Parabolic Type. American Mathematical Society, 1968.

[23] L.D. Landau, E.M. Lifschitz. Statistische Physik. Akademie Verlag, 1987.

[24] L.D. Landau, E.M. Lifschitz. Hydrodynamik. Akademie Verlag, 1991.

[25] W.J. Layton, F. Schieweck, I. Yotov. Coupling fluid flow with porous media flow. SIAM J. Numer. Anal., 40 (2003), $2195-2218$.

[26] R. Lipton, M. Avellaneda. Darcy's law for slow viscous flow past a stationary array of bubbles. Proc. Royal Soc. Edinburgh, 114A (1990), 71-79.

[27] A. Marciniak-Czochra, M. Ptashnyk. Derivation of a macroscopic receptor-based model using homogenisation techniques. SIAM J. Math. Anal., 40 (2008), 215-237.

[28] M. Neuss-Radu. Some extensions of two-scale convergence. C. R. Acad. Sci. Paris, 332 (1996), 899-904.

[29] G. Nguetseng. A general convergence result for a functional related to the theory of homogenization. SIAM J. Math. Anal., 20 (1989), 608-623.

[30] X.Y. Ni, T. Drengstig, P. Ruoff. The Control of the Controller: Molecular Mechanisms for Robust Perfect Adaptation and Temperature Compensation. Biophys. J., 97 (2009), 1244-1253.

[31] P. Nobel. Physicochemical EG Environmental Plant Physiology. Academic Press, 1999.

[32] M. Prosi, P. Zunino, K. Perktold, A. Quarteroni. Mathematical and numerical models for transfer of low-density lipoproteins through the arterial walls: a new methodology for the model set up with applications to the study of disturbed lumenal flow. J. Biomech., 38 (2005), 903-917.

[33] M. Ptashnyk. Derivation of a macroscopic model for nutrient uptake by a single branch of hairy-roots. Nonlinear Anal. Real World Applic., 11 (2010), 4586-4596.

[34] A. Quarteroni, M. Discacciati. Navier-Stokes/Darcy Coupling: Modeling, Analysis, and Numerical Approximation. Rev. Mat. Comput., 22 (2009), 315-426.

[35] E. Steudle, C.A. Peterson. How does water get through roots? J. Exp. Bot., 49 (1998), 775-788.

[36] E. Steudle. Water uptake by plant roots: an integration of views. Plant Soil, 226 (2000), 45-56.

[37] N. Sun, N.B. Wood, A.D. Hughes, S.A.M. Thom, X.Y. Xu. Effects of transmural pressure and wall shear stress on LDL accumulation in the arterial wall: a numerical study using a multilayered model. Am. J. Physiol. Heart. Circ. Physiol., 292 (2007), H3148 - H3157.

[38] L. Tartar. Incompressible fluid flow in a porous medium - convergence of the homogenization process. Appendix in Lecture Notes in Physics 127, Springer, Berlin, 1980.

[39] R. Temam. Navier-Stokes equations. North-Holland, Amsterdam, 1978.

[40] M. T. Tyree. The Thermodynamics of Short-distance Translocation in Plants. J. Exp. Bot., 20 (1969), 341-349.

[41] M. T. Tyree. The Cohension-Tension theory of sap ascent: current controversies. J. Exp. Bot, 48 (1997), $1753-1765$.

[42] A. Vailati, M.Giglio. Nonequilibrium fluctuations in time-dependent diffusion processes. Phys. Rev. E, 58 (1998), 4361-4371.

[43] J. Vos, J.B. Evers, G.H. Buck-Sorlin, B. Andrieu, M. Chelle, P.H.B. de Visser. Functional-structural plant modelling: a new versatile tool in crop science. J. Exp. Botany, 61 (2010), 2101-2115. 